\title{
Discrete Multitone Modulation for VSR and MR Electrical Interconnects and Optical Links
}

by

\author{
Hazem Beshara, BASc. Electrical Engineering, BSc. Computing \\ Technology
}

\author{
A thesis submitted to the \\ Faculty of Graduate and Postdoctoral Affairs \\ in partial fulfillment of the requirements for the degree of
}

Master of Applied Science in Electrical Engineering

Ottawa-Carleton Institute for Electrical and Computer Engineering

Department of Systems and Computer Engineering

Carleton University

Ottawa, Ontario

August, 2020

(C) Copyright

Hazem Beshara, 2020 


\section{Abstract}

A rich modulation scheme based transceiver is designed and implemented with throughput and error rate being given a high priority in order to target the continued exponential growth in the demand of data traffic ranging from intrachip high speed busses to metropolitan scale data center interconnects. A thorough analysis of the selection of key design details is performed both through analytical derivations and measurements that have not been previously done. Measurements using existing repurposed hardware are obtained in both an electrical very short to medium reach interconnect and an optical loop-back channel both containing a channel loss of 18dB. In conjunction to that, this work merges optimization techniques and introduces some that has not been used in previous work.

This resulted in a transceiver achieving $250 \mathrm{Gbps}$, an average of $3.5 \times 10^{-4} \mathrm{BER}$, a spectral efficiency of $5.8 \mathrm{~b} / \mathrm{s} / \mathrm{Hz}$, and an estimated power consumption of $5.1 \mathrm{pJ} / \mathrm{b}$, qualifying it be superior to prior art as well as suitable for both low power and high throughput applications in both coherent and non-coherent flavours of transceivers. It also serves as a blueprint for applying and adapting these optimization methods to varying design architectures, limitations of the analog circuitry, and frequency response of the system. 
This is dedicated to my incredibly supportive, talented, and brilliant family namely my parents Sherif and Mona and my brothers Sam and Ramy. You are an endless source of encouragement, inspiration, and advice.

"Family is a life jacket in the stormy sea of life"

- J.K. Rowling 


\section{Acknowledgments}

A great deal of appreciation goes to Dr. Calvin Plett for his supervision, support, deep technical and theoretical expertise, and efforts throughout this project. From accepting to taking me under his wing to his heavy involvement in a the project, only go to show the care he has for his students and genuine curiosity and enthusiasm of navigating technical challenges and discussing theory. Equally as such, a thank you to Dr. Naim Ben-Hamida who is always prepared to provide his guidance and input on the project ranging from high-level concepts to intricate details. His breadth and depth of knowledge is likely unsurpassed in the industry.

A thank you to Ciena Corporation for the opportunity to perform this project with access to state of the art equipment and exposure to brilliant individuals. Their dedication to the field of research is priceless to the development of young and upcoming engineers and to the telecommunications industry as a whole.

An acknowledgement goes out to the following colleagues from Ciena/Carleton for their help on either lab equipment, information on Ciena hardware, or their feedback on various aspects of the project : Zhe Jiang, Matt Mikkelsen, Jacob Pike, David Berton, Doug Charlton, Kristian Zanette, Ahmad Abdo, James Falt, Stewart Clelland, Jerry Lam, Sadok Aouini, and Mahdi Parvizi. 


\section{Table of Contents}

Abstract $\quad$ ii

Acknowledgments $\quad$ iv

Table of Contents $\quad$ v

List of Tables vii

List of Figures viii

Nomenclature $\quad$ x

1 Introduction $\quad 1$

1.1 Motivation ........................ 1

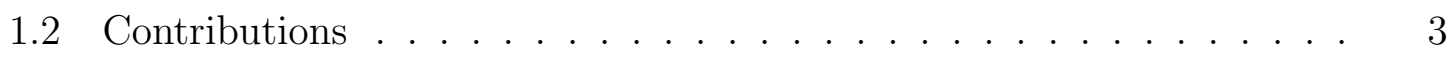

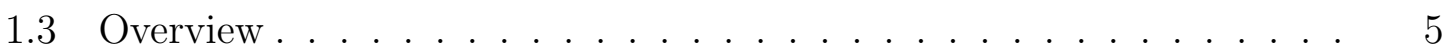

2 Background $\quad 6$

2.1 Transceivers . . . . . . . . . . . . . . . . . 6

2.1.1 Coherent vs Non-Coherent . . . . . . . . . . . . 6

2.2 Modulation ........................ . . 8

2.2.1 Pulse-Amplitude Modulation . . . . . . . . . . . 8

2.2.2 Orthogonal Frequency Division Multiplexing \& Discrete MultiTone Modulation . . . . . . . . . . . . . . . 10

2.3 Impairments . . . . . . . . . . . . . . . . . . . . . . . 15

2.3.1 Inter-Symbol Interference . . . . . . . . . . . . . . . 15

2.3.2 Frequency-Selective Response . . . . . . . . . . . . . . . . . . 17

2.3.3 Quantization Noise ............... 17 
2.3.4 Clipping Noise . . . . . . . . . . . . . . . . . . . . 18

2.3 .5 Overall SNR . . . . . . . . . . . . . . . . . . 20

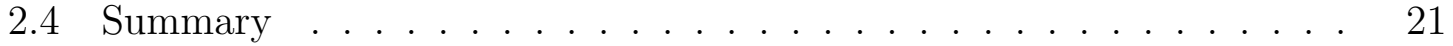

3 Design Considerations $\quad 22$

3.1 Channel Number Selection . . . . . . . . . . . . . . . . . . 23

3.2 Oversampling . . . . . . . . . . . . . . . . . . . . 30

3.3 Crest Factor and Clipping . . . . . . . . . . . . . . . . . . 32

3.4 Bit-Loading . . . . . . . . . . . . . . . . . . . . . . . 33

3.5 Efficient Fourier Transform . . . . . . . . . . . . . . . . . . 34

3.6 Synchronization . . . . . . . . . . . . . . . . . . . 35

3.7 Pre and Post Compensation . . . . . . . . . . . . . . 37

3.8 Summary . . . . . . . . . . . . . . . . . . . . 40

4 Measurements \& Results $\quad 42$

4.1 Electrical System . . . . . . . . . . . . . . . . . . 43

4.1 .1 System Description . . . . . . . . . . . . . . . . . . 43

4.1 .2 Transmitter . . . . . . . . . . . . . . . 44

4.1 .3 Receiver . . . . . . . . . . . . . . . . . . 46

4.1 .4 Results . . . . . . . . . . . . . . . . . . 47

4.2 Optical System $\ldots \ldots \ldots \ldots$

4.2 .1 System Description . . . . . . . . . . . . . . . . . 49

4.2 .2 Results . . . . . . . . . . . . . . . . . . 52

4.3 Power Consumption Estimate . . . . . . . . . . . . . . . . 54

4.4 Summary . . . . . . . . . . . . . . . . . . . . . . 59

5 Conclusion $\quad 60$

5.1 Trade-Offs Discussion . . . . . . . . . . . . . . . . . 62

5.2 Future Work . . . . . . . . . . . . . . . . . . 63

$\begin{array}{ll}\text { List of References } & 65\end{array}$

Appendix A Efficient Simultaneous FFT of Real Signals 69

Appendix B Efficient Implementation of Simultaneous FFT 


\section{List of Tables}

2.1 State of the art PAM based coherent transceivers . . . . . . . . 10

3.1 Summary of Design Points for Implemented DMT Systems (Electrical Channel and Optical Channel) . . . . . . . . . . . . . . . . 41

4.1 Implemented Electrical DMT System Power Consumption Estimate . 58

4.2 Summary of Measurement Performance Metrics . . . . . . . . . . 59

5.1 Performance summary and comparison of this work to published electrical-channel DMT . . . . . . . . . . . . . . . . . . . 62

5.2 Performance summary and comparison of this work to published optical-channel DMT . . . . . . . . . . . . . 63 


\section{List of Figures}

1.1 Interconnect Worldwide Growth Forecast [1] . . . . . . . . . . . . . 2

1.2 Data Center Interconnect Hierarchy $[2] \ldots$. . . . . . . . . . . . . 3

2.1 Conventional Coherent Transceiver Block Diagram . . . . . . . . . . 6

2.2 IMDD Transceiver Block Diagram . . . . . . . . . . . . . . . 7

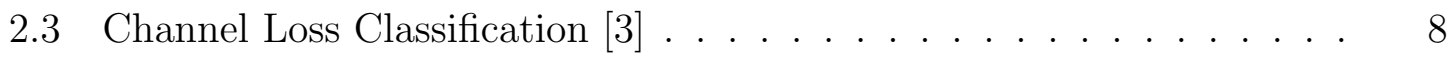

2.4 Standards for 112 Gbps Interconnects [4] . . . . . . . . . . . . . 9

2.5 Pulse Amplitude Modulation [5] . . . . . . . . . . . . . . . . 9

2.6 DMT Transmitter Block Diagram . . . . . . . . . . . . . . . . 12

2.7 DMT Receiver Block Diagram . . . . . . . . . . . . . . . . 13

2.8 Instantaneous amplitude of DMT symbol and its probability distribution 14

2.9 RC Effect on Square Pulse [5] . . . . . . . . . . . . . . . . 15

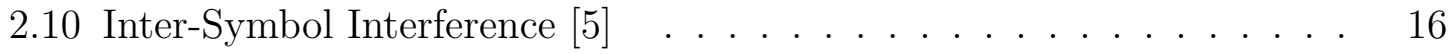

2.11 Cyclic Prefix . . . . . . . . . . . . . . . . 16

2.12 SNR clip vs Crest Factor $(\mu) \ldots \ldots \ldots$. . . . . . . . 19

2.13 SNR Total vs $\mu \ldots \ldots \ldots$. . . . . . . . . . . . . . . 21

3.1 DMT Design Tx Block Diagram . . . . . . . . . . . . . . . . . 22

3.2 DMT Design Tx Block Diagram . . . . . . . . . . . . . . . 23

3.3 Channel Number vs Measured PAPR Cumulative Distribution Function 24

3.4 SNR vs Crest Factor for different Clipping Amplitude . . . . . . . . . 24

3.5 Power Consumption of different FFT Length Modes [6] . . . . . . . 25

3.6 Frequency Scaled Power Consumption of different FFT Length Modes 26

3.7 BER for Different Sub-Channel Number Schemes (Full Spectrum) . . 27

3.8 BER for Different Sub-Channel Number Schemes (Lower Half Spectrum) 28

3.9 Mean Complex Distortion Vector for 128-Channel Scheme . . . . . . 29

3.10 Mean Complex Distortion Vector for 512-Channel Scheme . . . . . . 29

3.11 Measured Data Rate and BER for Various Fractional Oversampling Ratios . . . . . . . . . . . . . . . . . . 31 
3.12 Fractional Oversampling Ratio Implementation . . . . . . . . . . . . 31

3.13 BER vs Crest Factor . . . . . . . . . . . . . . . . . . . . . 33

3.14 Bit Loading . . . . . . . . . . . . . . . . . . . . . . . . 34

$3.15 \mathrm{Tx}$ and Rx Cross-Correlation . . . . . . . . . . . . . . . 36

3.16 Comparing BER for Precompensated vs Non-Precompensated for 128 Sub-Channels Scheme . . . . . . . . . . . . . . . . 38

3.17 Comparing BER for Precompensated vs Non Pre-compensated for 512 Sub-Channels Scheme . . . . . . . . . . . . . . . . . . . . . . 38

3.18 Comparing BER for Post compensated vs Non Post-compensated for 128 Sub-Channels Scheme . . . . . . . . . . . . . . . . 39

3.19 Comparing BER for Post compensated vs Non Post-compensated for 512 Sub-Channels Scheme . . . . . . . . . . . . . . . . . . . 40

4.1 Block Diagram of Electrical System Measurement Setup . . . . . . . 43

4.2 Evaluation Board indicating ADC/DAC Integrated Circuit and Interposer (yellow box) . . . . . . . . . . . . . . . . 44

4.3 Electrical System Overall Magnitude Response . . . . . . . . . . . . . 45

4.4 Electrical System Overall Phase Response . . . . . . . . . . . . . . . 45

4.5 Electrical System Transmitted Spectrum . . . . . . . . . . . . . . . . 46

4.6 Electrical System Received Spectrum . . . . . . . . . . . . . . . . . . 47

4.7 Electrical System BER Plot . . . . . . . . . . . . . . . . . . 48

4.8 Rx Constellation Electrical System Ch 1004.4 GHz) . . . . . . . . . 49

4.9 Rx Constellation Electrical System Ch $736(32.9 \mathrm{GHz})$. . . . . . . . 50

4.10 Rx Constellation Electrical System Ch $850(38.0 \mathrm{GHz})$. . . . . . . . 50

4.11 Rx Constellation Electrical System Ch 959 (42.9 GHz) . . . . . . . 51

4.12 Rx Constellation Electrical System Ch 1006 (45.0 GHz) . . . . . . . . 51

4.13 Optical System Test Setup Block Diagram . . . . . . . . . . . . . 53

4.14 Optical System Overall Magnitude Response . . . . . . . . . . . . . . 53

4.15 Optical System Overall Phase Response . . . . . . . . . . . . . . 54

4.16 Optical System BER Plot . . . . . . . . . . . . . . 55

4.17 Rx Constellation Optical System Ch $25(25.5 \mathrm{GHz})$. . . . . . . . . . 55

4.18 Rx Constellation Optical System Ch $75(16.0 \mathrm{GHz})$. . . . . . . . . 56

4.19 Rx Constellation Optical System Ch 95 (20.2 GHz) . . . . . . . . . 56

4.20 Rx Constellation Optical System Ch 105 (22.6 GHz) . . . . . . . . 57

4.21 Rx Constellation Optical System Ch $125(25.0 \mathrm{GHz}) \quad$. . . . . . . . 57 


\title{
Nomenclature
}

\author{
ADC Analog to Digital Converter \\ AFE Analog Front End \\ AGC Automatic Gain Control \\ BER Bit Error Rate \\ $\mathrm{b} / \mathrm{s} / \mathrm{Hz}$ Bits per Second per Hertz \\ DAC Digital to Analog Converter \\ CDR Clock and Data Recovery \\ CMOS Complementary Metal Oxide Semiconductor \\ CP Cyclic Prefix \\ CTLE Continuous Time Linear Equalizer \\ CW Continuous Wave \\ DFE Decision Feedback Equalizer \\ DMT Discrete Multitone Modulation \\ DSP Digital Signal Processing \\ EDFA Erbium-Doped Fiber Amplifier \\ ENOB Effective Number of Bits \\ FEC Forward Error Correction \\ FFE Feed Forward Equalizer \\ FFT Fast Fourier Transform \\ FIR Finite Impulse Response \\ Gbps Gigabit per Second \\ GS/s Giga-Sample per Second HD-FEC Hard Decision FEC \\ IFFT Inverse Fast Fourier Transform \\ IMDD Intensity Modulation Direct Detection \\ IP Internet Protocol \\ ISI Inter-Symbol Interference \\ LR Long Reach OBPF Optical Band-Pass Filter
}


OFDM Orthogonal Frequency Division Multiplexing

PAPR Peak to Average Power Ratio

PAM Pulse Amplitude Modulation

PBS Polarization Beam Splitter

PD Photo-Diode

$\mathrm{pJ} / \mathrm{b}$ Picojoule per Bit

PLL Phase-Locked Loop

PR Phase Rotator

MR Medium Reach

SD-FEC Soft Decision FEC

SNR Signal to Noise Ratio

SQNR Signal to Quantization Noise Ratio

TIA Trans Impedance Amplifier

Tbps Terabit per Second

$\mu$ ITLA Micro Integrable Tunable Laser Assembly

VGA Variable Gain Amplifier

VOA Variable Optical Attenuator

VSR Very Short Reach 


\section{Chapter 1}

\section{Introduction}

\subsection{Motivation}

The continuously increasing demand for higher data rates and more bandwidth across multiple applications, such as delivering multimedia content to end users, connecting data centers, and interconnecting high-speed components on devices has been the driver behind many of the advances in the telecommunications industry [7]. As cities and rural areas grow in size, density, and population, more devices are placed on networks, this demand is predicted to grow exponentially. Ethernet standards have already reached 400 Gbps standards and are predicted to reach 800 Gbps within the next couple of years [8]. This demand is also accompanied by the need to reduce overall power consumption for cost reduction and environmental reasons, reduce cost of development and manufacturing, as well as a reduction in complexity. This work will address two of the critical applications of high speed transceivers. The first application being data center interconnects (DCIs).

As networks become more critical to everyday activities, it has become vital to bring cloud computing and data centers away from legacy infrastructure of being centralized and towards a distributed edge node model. This has the advantage of lowering the latency to the end user as the data is physically closer and allows redundancy and backups. The traffic is not only limited to typical multimedia, but also extends to vital infrastructure such as banking, tele-medicine, education, national security, self-driving vehicles, and collaboration for businesses. A direct consequence of this is the exponential growth in interconnection bandwidth required to seamlessly integrate all these nodes into the world wide network. Equinox forecasts that by 2022, 


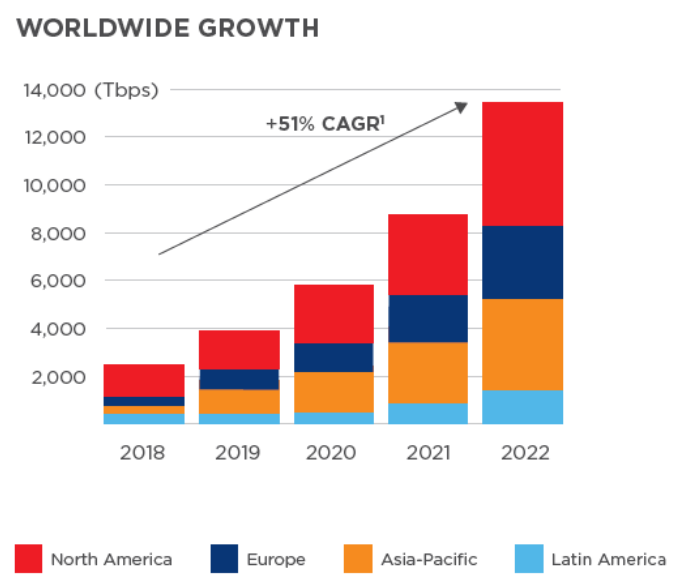

Figure 1.1: Interconnect Worldwide Growth Forecast [1]

global interconnect bandwidth will have seen a $51 \%$ growth to 13,300 Tbps [1].

Due to this exponential growth in demand, the desire to drastically increase throughput capabilities is a primary goal of the systems interconnecting data centers and cloud computing nodes. There is of course the need to achieve these high speeds at a lower power consumption due to the scale of the number of devices across all these centers, but the throughput remains the critical targeted metric. As of this writing, the highest standard for Ethernet according to the OIF Implementation Agreement is 400Gbps. Industry devices can achieve this over a single wavelength of light, and have even brought to market $800 \mathrm{Gbps}$ capable devices. The distance of these links ranges from a few kms to a few hundred kms.

The second application to be targeted is backplane interconnects, whereby the endpoints of data center interconnects branch out to various racks and connect networking devices within a rack to one another and between racks as seen in Figure 1.2. These are high speed links that physically reside within 0.5-3 meters of one another. These circuits come in small form factors (few $\mathrm{cms} \mathrm{x}$ few $\mathrm{cms}$ ) and have stringent power requirements due to their size and high quantities within data centers. These standards for pluggables ranges from 50 Gbps all the way up to 400 Gbps.

A significant portion of the efforts to address those demands is done through pre-processing and post-processing the data being transmitted to target either power reduction, maximizing throughput, minimizing errors in transmission, or address key degradation that occurs to the data that is specific to the application. 


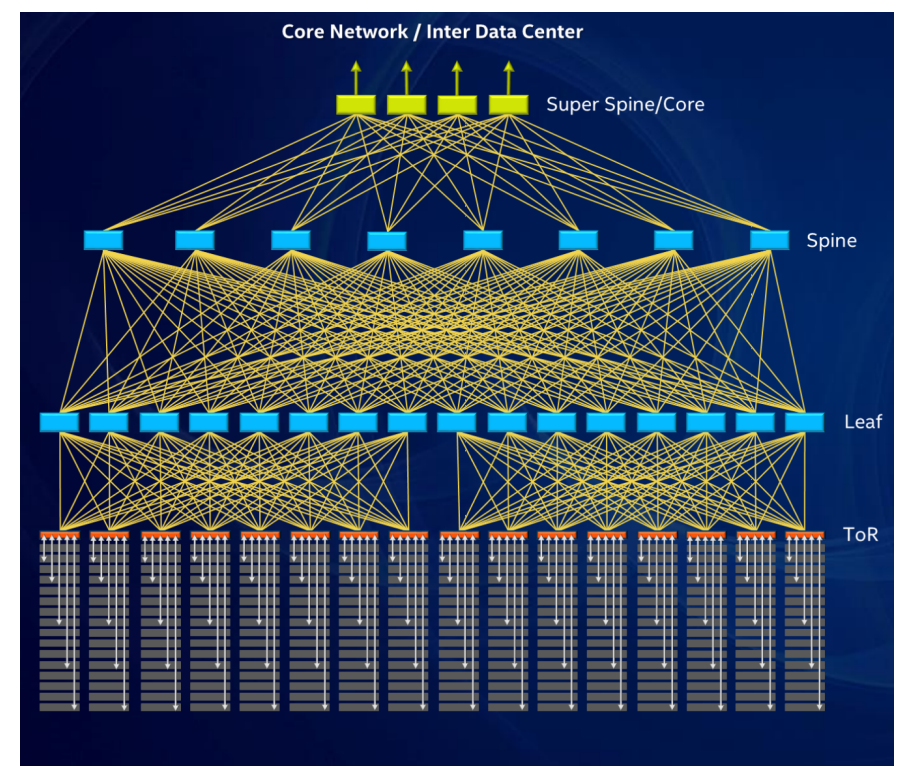

Figure 1.2: Data Center Interconnect Hierarchy [2]

A critical aspect of any wireline/wireless telecommunications system, is the modulation scheme of the data. The modulation scheme will determine how complex the transceivers are, how resilient they are to different types of impairments, what data rates the system can achieve at a particular bit error rate, and the amount of power they consume. This work explores the modulation scheme known as Discrete Multitone Modulation (DMT), which is not the convention for current state of the art transceivers. The reason for that is likely the fact that it's more difficult to standardize such a versatile modulation scheme, in addition to the requirement of a high resolution and sampling frequency of the data converters. Another possible reason is that there wasn't enough analysis and measurements performed to demonstrate the true capabilities of DMT and dispel some of the uncertainties around any possible drawbacks. One such drawback is the high linearity required, which will be addressed in this work.

\subsection{Contributions}

The contributions of this work are as follows :

- The novelty of using fractional oversampling of DMT signals

- The merging of the following optimization techniques in specific order to ensure 
optimal performance. Each of them have been discussed in prior work, but their merging has yet to be demonstrated to provide a complete design methodology.

- Sub-channel width Selection to maximize resiliency against frequency varying response

- Oversampling - in particular how fractional oversampling has a tremendous benefit over integer oversampling

- Maximizing signal to noise ratio (SNR) using optimal amplitude clipping. This is compared against a total SNR equation derived with quantization, clipping, and thermal noise considered in terms of clipping amplitude relative to the variance of the DMT signal

- Using bit-loading to maximize sub-channel utilization and overall throughput

- Efficient resource utilization through the use of simultaneous fast Fourier transform (FFT)

- Frequency response compensation using frequency domain postcompensation

- A DMT based transceiver with the following performance metrics

- 250 Gbps throughput for an 18dB loss electrical interconnect, which is higher than published work in this space

- $5.82 \mathrm{~b} / \mathrm{s} / \mathrm{Hz}$ spectral efficiency, which is $45 \%$ higher than published work

- $5.1 \mathrm{pJ} / \mathrm{b}$ estimated power consumption, which is approximately a third that of rivaling conventional transceivers

- Trade-offs are evaluated and highlighted to form a blueprint for targeting the DMT system at applications that have different requirements

- At the time of writing, a publication has been submitted to ISSCC 2021 and a patent application is process with Ciena Corporation 


\subsection{Overview}

This thesis is organized as follows. Chapter 2 introduces concepts around conventional transceivers and modulation, It also describes the modulation scheme to be used for this work and highlights its differences to that of conventional systems. Chapter 3 provides the detailed design of the implemented system, which includes various novel aspects due to the combination of various optimization techniques and the introduction of a novel technique not previously used in DMT. Chapter 4 describes the equipment in which the DMT system was implemented, measurement results, and a power consumption estimate. Chapter 5 concludes the findings, achievements, and contributions in this work while proposing ideas and possible improvements for future work. 


\section{Chapter 2}

\section{Background}

\section{$2.1 \quad$ Transceivers}

This chapter aims to deliver some basic background knowledge on types of transceivers and conventional methods of modulating data - providing context as to how this work differs from what's widely used in today's transceivers. It then introduces the concept of DMT, which will be broken down further in details throughout Chapter 3.

\subsubsection{Coherent vs Non-Coherent}

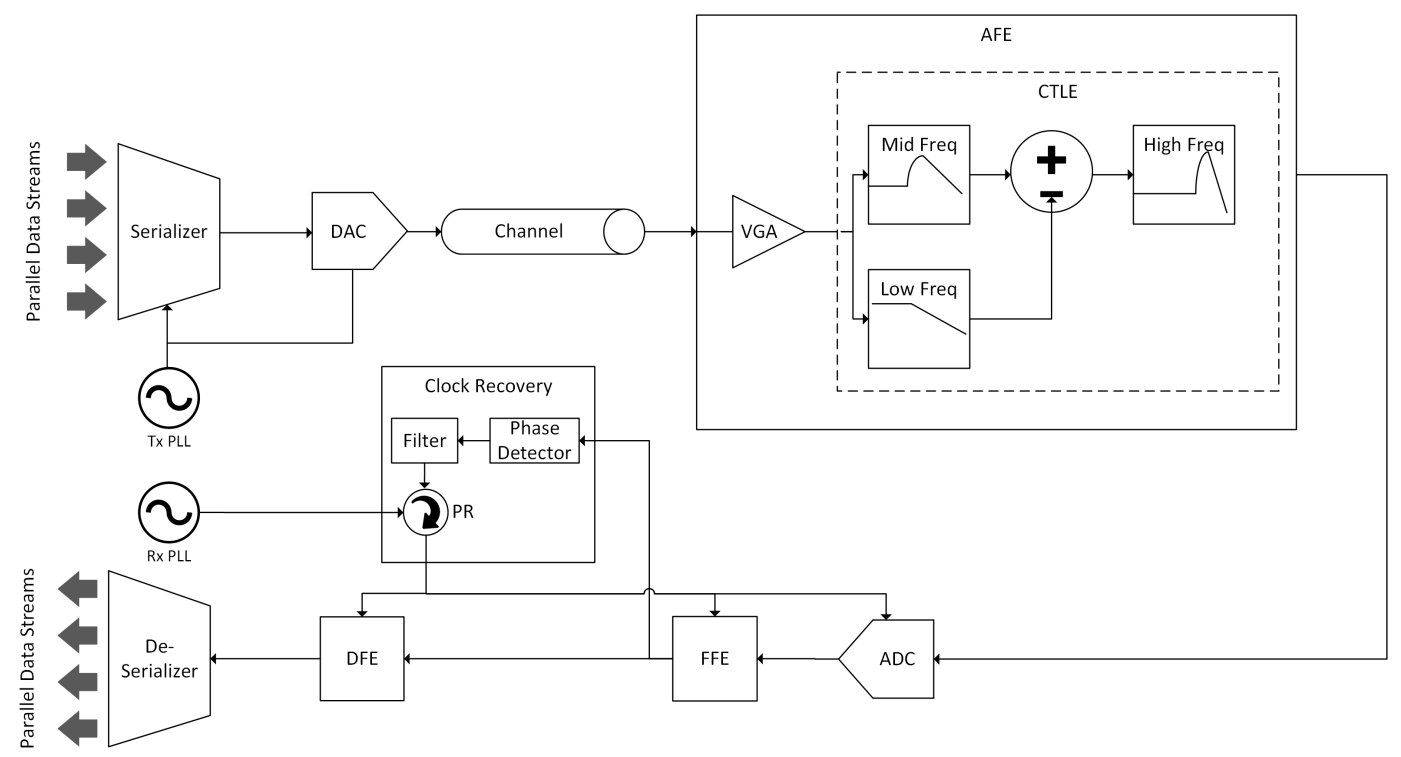

Figure 2.1: Conventional Coherent Transceiver Block Diagram 
A coherent transceiver is characterized by the need to recover the clock used for transmission in order to demodulate the signal. Figure 2.1 shows an overview of a conventional coherent transceiver indicating the transmitter, analog front end (AFE) of the receiver, clock and data recovery, and equalization blocks [9] [10] [11] [12] [13] [14]. Coherent systems allow for sensitivity improvements at the receiver side at the cost of an increased complexity and power consumption. Non-coherent transceivers, which can be referred to as direct-detection, on the other hand do not require clock recovery in order to recover the transmitted data. Figure 2.2 shows the typical architecture for an intensity modulated direct detection transceiver (IMDD) [15] [16] [17] [18].

To the author's knowledge, transceivers for electrical channels typically use CDRs, whereas IMDD has been explored in optical links in recent publications.

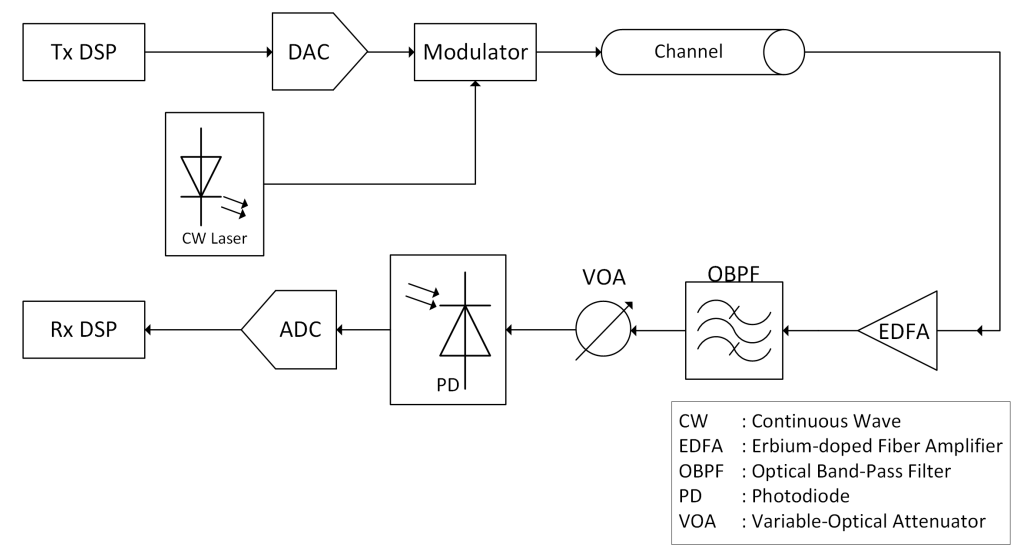

Figure 2.2: IMDD Transceiver Block Diagram

This thesis will explore the technique of discrete multi-tone modulation in both a coherent and a non-coherent transceiver. More specifically, the concept of DD will be experimented within an electrical channel, and a coherent system will be implemented for an optical link.

The medium in which the signal traverses, or channel, introduces impairments onto the signal that need to be corrected for in order to have a reasonably reliable link. Two of these main impairments are attenuation and inter-symbol interference (ISI), details of which will be discussed in section 2.3.1. Figure 2.3 shows how attenuation increases with increasing frequencies for various types of links, and Figure 2.4 shows the current standards for various 112 Gbps interconnects. 


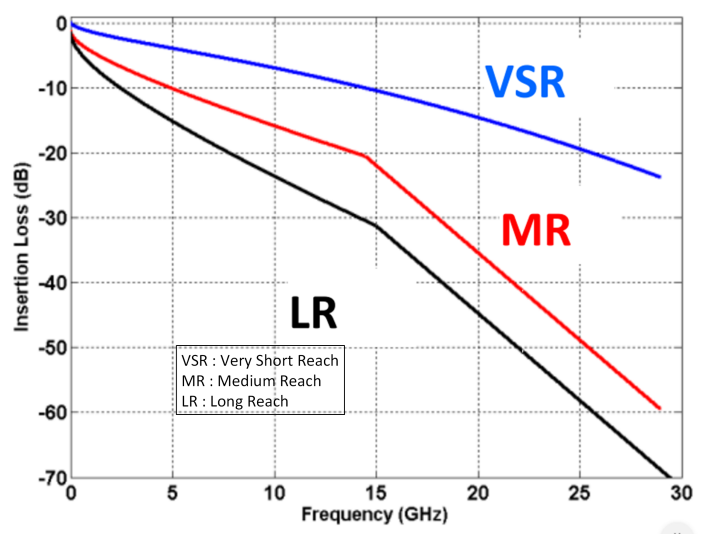

Figure 2.3: Channel Loss Classification [3]

Typical wireline transceivers use equalizers in order to address these two aforementioned impairments, where the design of these equalizers takes up a considerable amount of effort. These equalizers fall into three categories: Feed-Forward Equalizer (FFE), Decision Feedback Equalizer (DFE), and Continuous Time Linear Equalizer (CTLE). The AFE will typically house one more variable gain amplifiers combined with one or more CTLEs that will compensate for the non-flat frequency response seen in Figure 2.3 by either boosting high frequency components or attenuating low frequency components (or both in multistage cases) in order to have a net frequency response that is flat. FFEs and DFEs are finite impulse response (FIR) filters that target two sub-types of ISI. They utilize delay elements, multipliers, and adders with various coefficients to counteract the effects of ISI. Conventional wireline transceivers also require clock recovery, which contains a phase detector, a loop filter, a phase rotator (or interpolator), and an external clock source such as a phase-locked loop (PLL).

\subsection{Modulation}

\subsubsection{Pulse-Amplitude Modulation}

Pulse amplitude modulation (PAM) is a technique where the signal space is split into $\mathrm{M}=2^{k}$ finite energy levels to represent $M$ distinct symbols, each symbol being a group of unique $\mathrm{k}$ bits. Conventional wireline transceivers utilize some form of PAM, such 


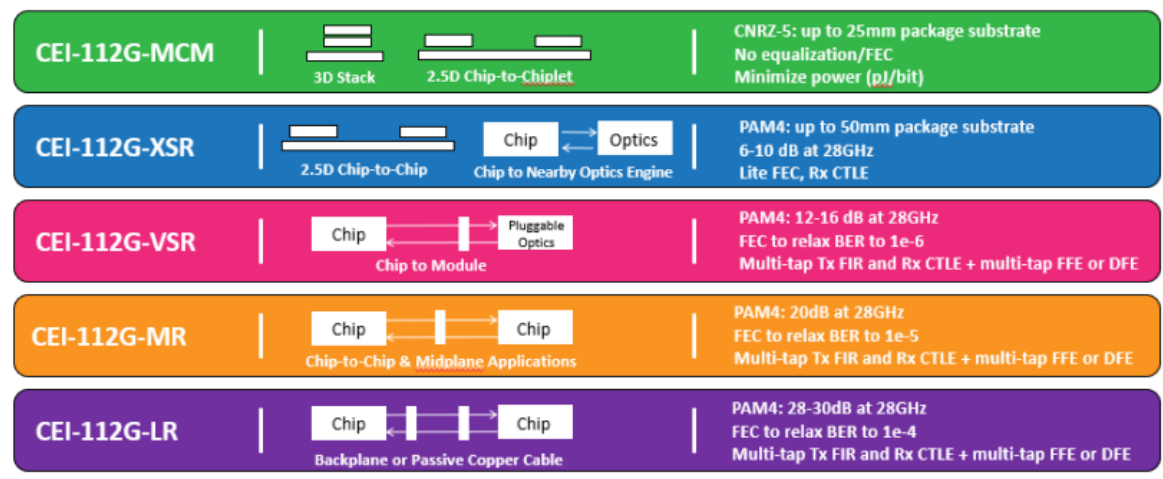

Figure 2.4: Standards for 112 Gbps Interconnects [4]

as PAM2 or PAM4 (the number corresponding to $\mathrm{k}$ ), or non-return-to-zero (NRZ). NRZ is the case where only two energy levels are used, neither of them being zero. This eliminates the need to deal with a DC component. Figure 2.5 demonstrates PAM-4 and PAM-8, where each symbol is represented by an energy level across a single axis.

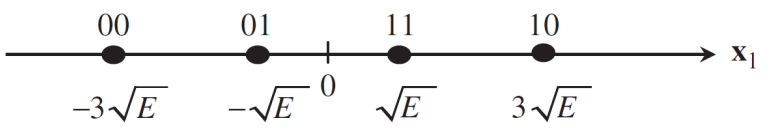

(a) $M=4$

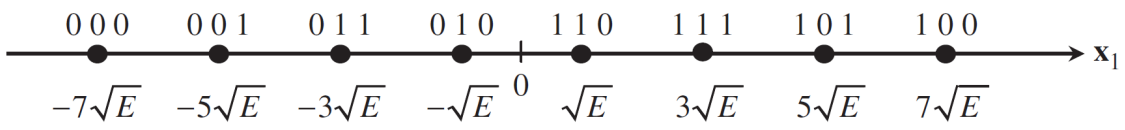

(b) $M=8$

Figure 2.5: Pulse Amplitude Modulation [5]

State of the art work in PAM coherent transceivers can be summarized in Table 2.1. In order to better compare conventional transceivers to the proposed design in this work, the power from the second transceiver from Table 2.1 will be considered, as it targets a similar channel loss as the one in this work (corresponding to a medium reach interconnect). The total power consumption gathered from the provided info is 1.57 watts, which includes transmitter, PLL and clock distribution, receiver, and ADC. A critical component not included in this power consumption quote is the DSP portion - making it cumbersome to the task of comparing conventional transceivers to the one designed in this work. However, this consumption figure will aid in the 
presentation of trade-offs between the proposed work and conventional designs from the perspectives of power and design complexity.

Table 2.1: State of the art PAM based coherent transceivers

\begin{tabular}{|l|l|}
\hline & {$[13][19]$} \\
\hline Throughput (Gbps) & 112 Gbps \\
\hline Modulation & PAM-4 \\
\hline & $\begin{array}{l}\text { CTLE } \\
31-\text { Tap FFE } \\
1-\text { Tap DFE }\end{array}$ \\
\hline Rx Equalization & $3.08 \mathrm{pJ} / \mathrm{b}$ \\
\hline Rx Power (excl. DSP) & $\begin{array}{l}\text { ADC: } 8.48 \mathrm{pJ} / \mathrm{S} \\
\text { RX: } 5.27 \mathrm{pJ} / \mathrm{b}\end{array}$ \\
\hline PLL and Clock Distribution & $1.47 \mathrm{pJ} / \mathrm{b}$ \\
\hline Channel Loss & $20 \mathrm{~dB}$ \\
\hline Pre-FEC BER & $2.00 \mathrm{E}-05$ \\
\hline
\end{tabular}

\subsubsection{Orthogonal Frequency Division Multiplexing \& Dis- crete Multi-Tone Modulation}

Orthogonal Frequency Division Multiplexing is a modulation scheme where the conventional single carrier aforementioned is replaced with multiple carriers spanning the available bandwidth that form $\mathrm{N}$ narrow subchannels that overlap in frequency but are orthogonal. Each of those subchannels may be independent from one another and can be modulated and manipulated individually to maximize a certain metric (throughput, BER, etc.). Discrete Multi-Tone Modulation is the baseband form of OFDM.

The need for having multiple analog paths (equal to the number of sub-carriers) is eliminated through the use of a digital circuit that can produce these orthogonal multiple carriers that span the desired bandwidth.

Figures 2.6 and 2.7 demonstrate the principles of OFDM/DMT. At the transmitter side, a high-speed binary bus is divided into N parallel lower speed busses. For each 
lower speed bus, M-quadrature amplitude modulation can be performed by grouping $\mathrm{m}$ bits, where $2^{M}$ represents the possible number of states and $m=0,1, \ldots, M-1$ represents the state of the grouped bits. Each state is then mapped to a complex symbol $X_{i}=A_{i}+j B_{i}$. A stream of complex symbols $X_{0} \ldots X_{k}$ is then converted to the time domain through the use of the Inverse Discrete Fourier Transform (IDFT), resulting in

$$
S_{n}=\sum_{k=0}^{N-1} X_{k} \exp \left\{\frac{j 2 \pi n k}{N}\right\}, n=0,1, \ldots, N-1
$$

In order to ensure that the output of the IDFT is real (as to avoid partial loss of data since only the real component can be transmitted), the input to the IDFT must satisfy Hermitian symmetry [20]. That results in 2.2 to be adjusted as follows :

$$
S_{n}=\sum_{m=0}^{2 N-1} x_{m} \exp \left\{\frac{j 2 \pi n m}{2 N}\right\}, n=0,1, \ldots, N-1
$$

where $X_{m}=\left\{X_{k}, X_{k}^{*}\right\}$ and $k=0,1, \ldots, N-1$

The inverse Fast-Fourier Transform (IFFT) is then used to modulate these parallel busses of complex symbols into $\mathrm{N}$ orthogonal subchannels. The output of the IFFT, which will be referred to as the DMT symbol throughout this work, is a stream of $\mathrm{N}$ orthogonal subchannels. Each DMT symbol is then appended with a cyclic prefix $(\mathrm{CP})$ to mitigate some of the impairments as well as synchronize between the transmitter and receiver. Details of the cyclic prefix are discussed in section 2.3.1. 


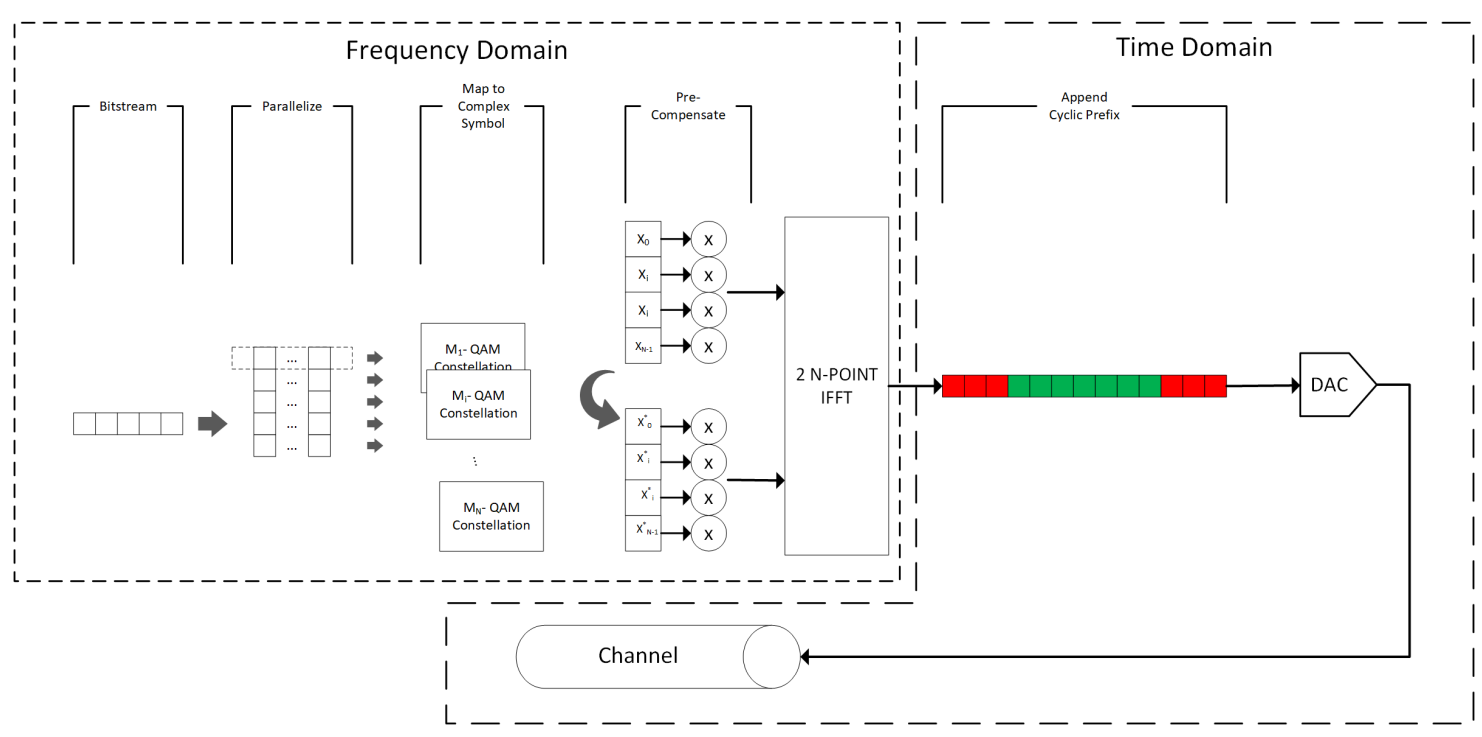

Figure 2.6: DMT Transmitter Block Diagram

The signal may then be passed to a frequency response compensation filter before being converted to an analog signal and applied onto the channel.

At the receiver, the received signal is converted to a digital stream that may be further frequency compensated in the case where the compensation so far has not been sufficient or in the case of splitting the compensation between the receiver and the transmitter. The considerations for how to split the compensation will be discussed in section 3.7. The cyclic prefix that had been appended at the transmitter is then removed before being fed through to the FFT in order to retrieve the original complex symbols $X_{i}$. $Y_{N}$ to $Y_{2 N}$ are dropped as they are mere complex conjugates of the originally transmitted $X_{0}$ to $X_{N-1}$. Each channel is demodulated according to its respective QAM order to produce the parallel lower speed buses. Finally, the parallel buses can be serialized to obtain the transmitted high speed bitstream. 


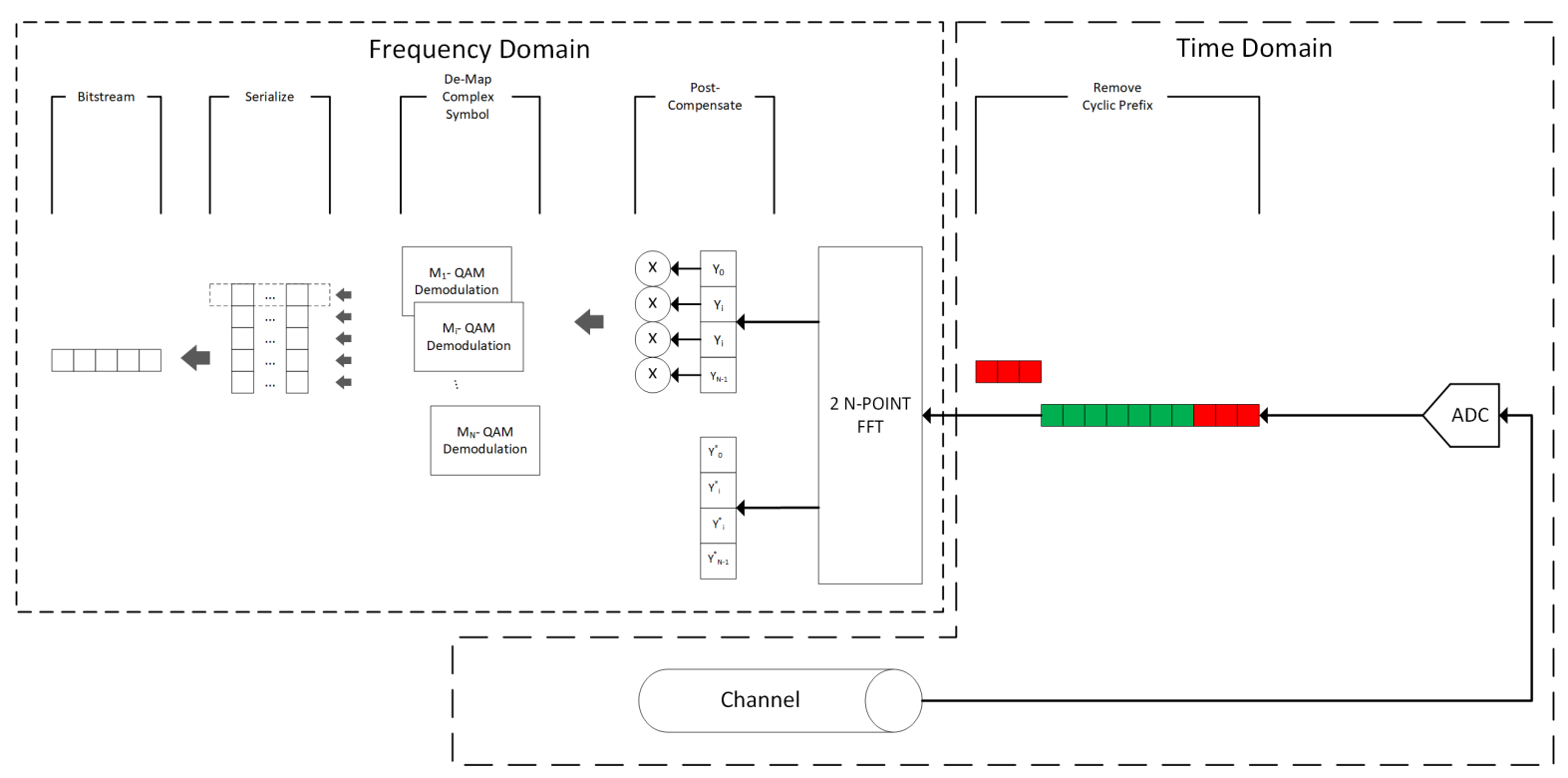

Figure 2.7: DMT Receiver Block Diagram

Various advantages arise due to using DMT. Firstly, deterministic impairments on individual channels can be compensated for in the frequency domain prior to DMT modulation. Secondly, channels can have different modulation orders in order to minimize the overall BER of the system.

In order to derive optimal configuration of various aspects of DMT modulation to maximize throughput while minimizing BER, an analytical model of a DMT signal is needed. Fundamentally, A DMT modulated signal is the sum of N independent QAM signals modulated on a distinct carrier [21]. As the number of channels is large, $N \geq 10$, the DMT time domain signal (output of the IFFT) $d(t)$ can be accurately modelled by a Gaussian random process with zero mean and a variance $\sigma^{2}$ as can be seen in Figure 2.8.

The probability $\mathrm{p}(\mathrm{x})$ that the instantaneous value of $\mathrm{d}(\mathrm{t})$ is $\mathrm{x}$ can be seen as follows

$$
p(x)=\frac{1}{\sigma \sqrt{2 \pi}} e^{\frac{-x^{2}}{2 \sigma^{2}}}
$$

where $\sigma$ is the standard deviation of the signal.

Due to the inherent nature of a DMT signal, in which it is comprised of multiple carriers, high instantaneous peaks may be produced. Up to $\mathrm{N}$ of these carriers can align in phase instantaneously, providing a peak that's $\mathrm{N}$ times as large as the amplitude of an individual carrier. To quantify this phenomena and its effects on 

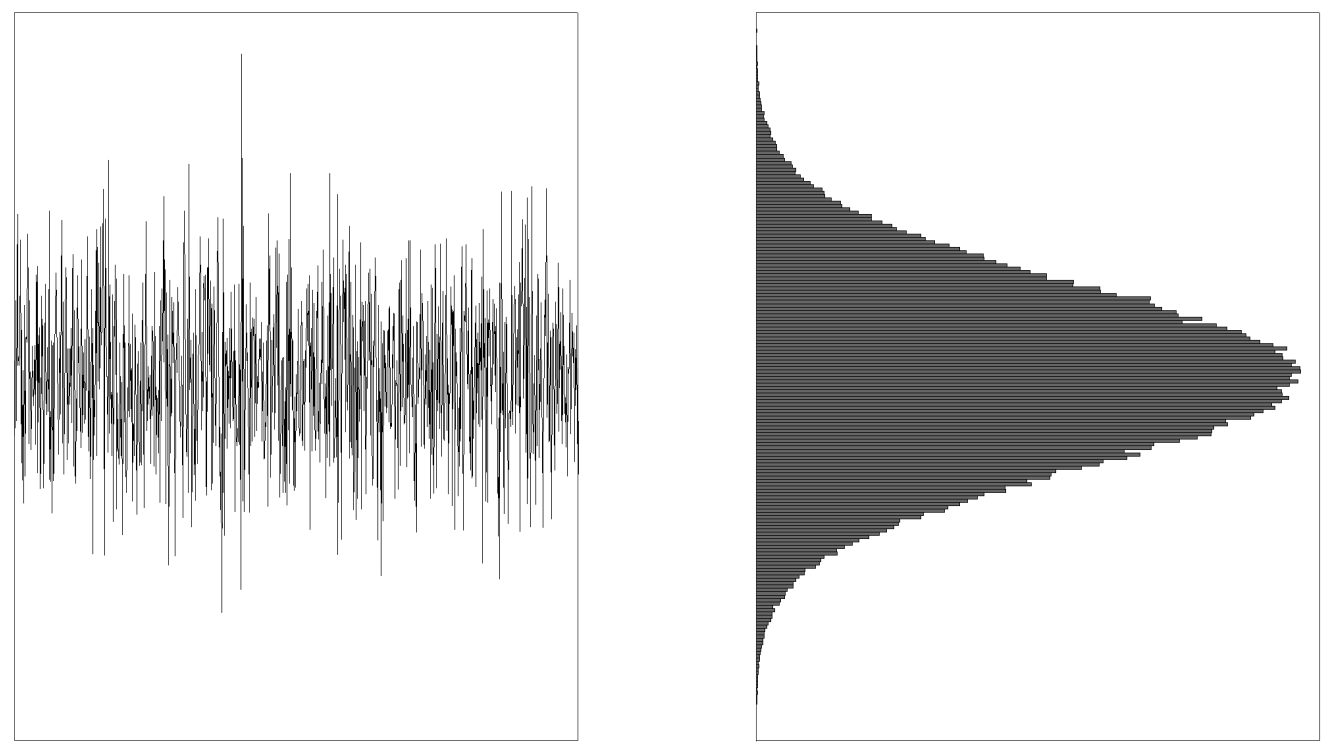

Figure 2.8: Instantaneous amplitude of DMT symbol and its probability distribution

performance, the crest factor (otherwise known as peak-to-rms ratio) can be defined as follows :

$$
\mu=\frac{a_{\text {peak }}}{s_{\text {rms }}}
$$

where $a_{\text {peak }}$ is the peak amplitude of the DMT signal and $s_{r m s}$ is the root-meansquare of the DMT signal. This can be re-written in terms of $\sigma$,

$$
\mu=\frac{a_{\text {peak }}}{\sigma}
$$

as the root-mean-square of a signal is equal to its variance.

If there is clipping being applied to the signal, then Equation 2.5 becomes :

$$
\mu=\frac{a_{\text {clip }}}{\sigma}
$$

References in this work to crest factor will be in terms of Equation 2.6, where clipping is taken into account.

Having a large crest factor has a number of disadvantages, including increased dynamic range and complexity of the converters (ADC and DAC), reduced efficiency 
of drivers and power amplifiers, and an increase in power consumption. Three categories of techniques can be used to reduce crest factor: signal distortion techniques, coding techniques, and scrambling techniques [22]. This work will explore the most straightforward of these, signal distortion techniques, in which clipping is applied to optimally reduce the crest factor for a given implementation.

\section{$2.3 \quad$ Impairments}

\subsubsection{Inter-Symbol Interference}

Inter-symbol interference is the result of the spreading of a symbol in the time-domain that interferes with adjacent symbols due to channel distortion, frequency selective magnitude and phase response, multi-path interference, and the bandlimited nature of a communication system. In a conventional transceiver, this is corrected for using equalizers at the transmitter and receiver that are often complex, consume a considerable amount of power, and take up significant area on a transceiver.

Consider a channel's response to simply be modeled using its equivalent low-pass response using an RC-filter as seen in Figure 2.9 and the effect it has on an input squared pulse signal.
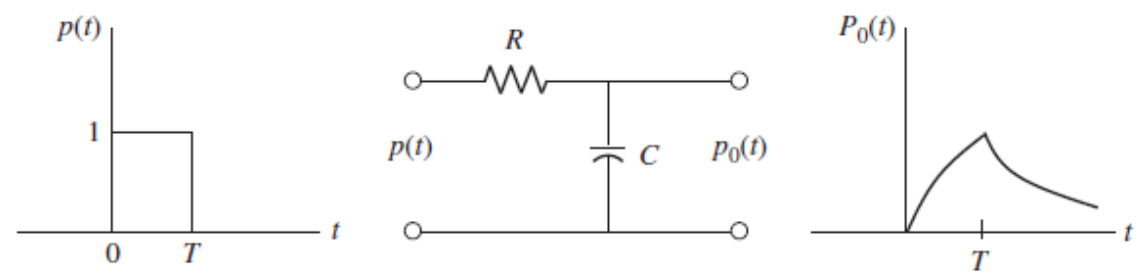

Figure 2.9: RC Effect on Square Pulse [5]

DMT uses a method of overcoming ISI that's simpler than the one used in conventional transceivers, known as Cyclic Prefix, whereby a guard interval is inserted between each DMT symbol. The ISI is a result of the limited bandwidth of the system in addition to group delay of the signal as it traverses from the transmitter to the receiver. The length of the guard interval must be larger than the symbol spread to avoid distorting the portion of the symbol to be used for recovering the transmitted 

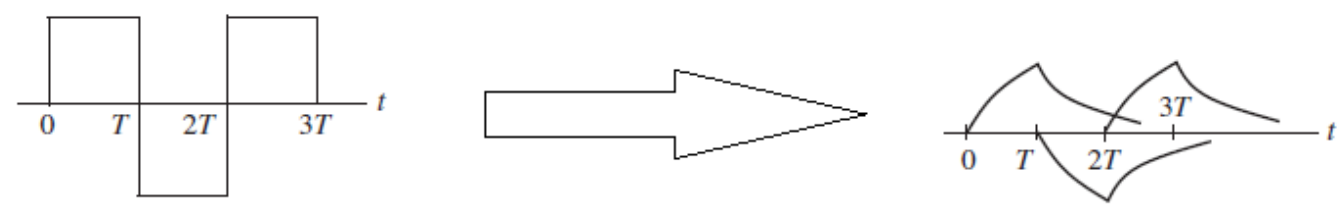

Figure 2.10: Inter-Symbol Interference [5]

data. Cyclic Prefix comes at the cost of throughput due to the overhead, whereby a percentage reduction in throughput is equivalent to the cyclic prefix percentage used expressed as a percentage of $S_{\text {partial }}$ as seen in Figure 2.11.

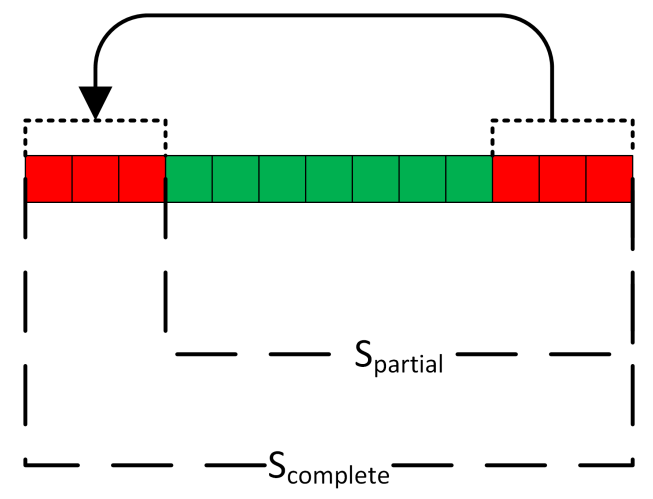

Figure 2.11: Cyclic Prefix

For optical communication systems, CP will also combat degradation phenomenons such as chromatic dispersion and polarization mode dispersion. Chromatic dispersion is the equivalent of group delay in an electrical channel, whereby different wavelengths of light propagate at different velocities and arrive at the destination at different moments in time. Polarization mode dispersion is caused by misalignment in polarization between the receiver and the transmitter.

An indirect benefit of using cyclic prefix is the improved capability of synchronizing between the transmitter and receiver in the case of non-coherent systems. This will be discussed further in Chapter 3 section where the synchronization technique used in this work will be discussed. 


\subsubsection{Frequency-Selective Response}

Due to the required throughput and the accompanying needed spectrum, and varying responses of the circuitry along the analog chain, there may be a large variation in the magnitude and phase response experienced by the transmitted signal at different frequencies. DMT is especially resilient to this as each sub-channel can be sufficiently narrow such that it experiences a flat frequency response. In addition to that, each sub-channel can be corrected for independently during pre-processing by applying a complex corrector factor to each sub-channel

\subsubsection{Quantization Noise}

The limited resolution at the front end of the transmit and receive chains (DAC/ADC) introduce quantization noise and presents a limitation on the upper bound of the modulation scheme for each sub-channel. Therefore, a critical aspect of the performance of a DMT system (throughput and BER in particular) is the resolution of both the $\mathrm{DAC}$ and the ADC.

From [23] a quantization step $\Delta$ of a converter with $\mathrm{n}$ bits is expressed as follows:

$$
\Delta=\frac{2 A_{\max }}{2^{b}}
$$

where the quantization noise power is [23] :

$$
P_{\text {quantization }}=\frac{\Delta^{2}}{12}
$$

which can be re-written in terms of the crest factor in terms of the clipping amplitude using 2.5 :

$$
\begin{aligned}
P_{\text {quant }_{c}} & =\frac{\Delta_{c}^{2}}{12} \\
& =\frac{2^{2} A_{\text {clip }}^{2}}{12 \cdot 2^{2 b}} \\
& =\frac{2^{2} \mu^{2} \sigma^{2}}{12 \cdot 2^{b}}
\end{aligned}
$$

Using 2.7 and 2.8 the SQNR (Signal-to-Quantization Noise Ratio) can be derived 
as follows :

$$
\begin{aligned}
S Q N R=\frac{S}{N_{Q}} & =\frac{\sigma^{2}}{P_{\text {quantization }}} \\
& =\frac{12 \cdot 2^{2 b-2}}{\mu^{2}}
\end{aligned}
$$

\subsubsection{Clipping Noise}

As shown in Section 2.2.2, DMT inherently suffers from a high peak-to-average power ratio, which has implications on the circuitry in the transmitter, which includes the DACs, modulators, drivers, power amplifiers, and any electro-optics used. These components have limited linearity and limited drive capability, which causes degradation in the signal and requires additionally complex components. Ultimately, a portion of the signal to be transmitted needs to be clipped, scaled, or a combination of the two in order to meet the specifications of the transmitter in terms of linearity and drive capability whilst minimizing BER.

The increase in PAPR will result in an increased BER as a result of either clipping the signal or scaling the signal to a point where thermal noise and quantization noise result in a decreased SNR of the transmitted signal. An apparent trade-off emerges as a result of the desire to increase the number of channels used to overcome large variations in the frequency response of the system. This allows a finer control over the modulation index of each channel depending on the SNR of various portions of the bandwidth used. However, this results in an increased PAPR as can be seen in equation 2.11 found in [24]. This thesis will explore this trade-off and highlight the analysis needed to find an optimal point.

$$
P A P R=\frac{\max |x(t)|^{2}}{\frac{1}{N T} \int_{0}^{N T}|x(t)|^{2}}
$$

Given a limited amplitude $A_{\text {clip }}$ and a DMT signal probability distribution function described by Equation 2.3 the total power of the clipped slice of a DMT symbol is [21]: 


$$
\begin{aligned}
P_{\text {clip }} & =2 \int_{A_{\text {clip }}}^{+\infty}\left(x-A_{\text {clip }}\right)^{2} \cdot \rho(x) d x \\
& =\sigma^{2}\left(-\sqrt{\frac{2}{\pi}} \mu \exp \frac{-\mu^{2}}{2}+\left(1+\mu^{2}\right) \operatorname{erfc}\left(\frac{\mu}{\sqrt{2}}\right)\right)
\end{aligned}
$$

The signal-to-clipping noise power ratio can be derived, considering $P_{\text {sig }}=\sigma^{2}$ :

$$
\begin{aligned}
S N R_{\text {clip }} & =\frac{P_{\text {sig }}}{P_{\text {clip }}} \\
& =\left(-\sqrt{\frac{2}{\pi}} \mu \exp \frac{-\mu^{2}}{2}+\left(1+\mu^{2}\right) \operatorname{erfc}\left(\frac{\mu}{\sqrt{2}}\right)\right)^{-1}
\end{aligned}
$$

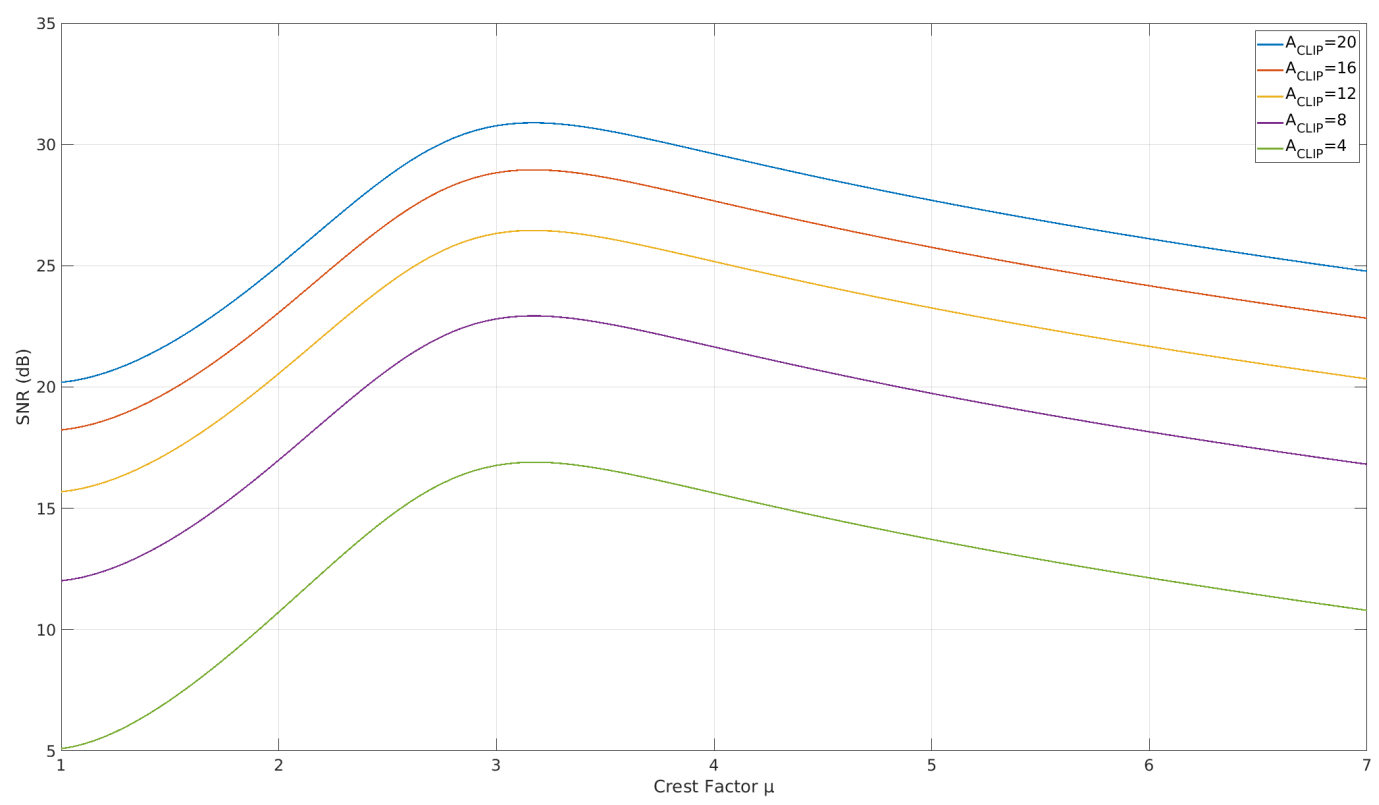

Figure 2.12: SNR clip vs Crest Factor $(\mu)$

Figure 2.12 indicates that as the clipping amplitude is increased, the SNR increases. However, the clipping amplitude cannot be set to an arbitrarily large value to maximize SNR. This is due to the intrinsic limited drive capabilities of the transmitter, in addition to the effects of quantization noise as will be demonstrated in the 
following section.

\subsubsection{Overall SNR}

In order to determine the optimal crest factor that would maximize SNR for a given case, a derivation of the total SNR is performed.

Considering thermal noise $P_{\text {therm }}$ is

$$
P_{\text {therm }}=k T B
$$

where $\mathrm{k}$ is the Boltzman constant, $\mathrm{T}$ is temperature in kelvin, and $\mathrm{B}$ is the bandwidth [25]. The overall SNR with quantization, clipping, and thermal noise taken into account can be seen as follows :

$$
S N R=\frac{P_{\text {sig }}-P_{\text {clip }}}{P_{\text {therm }}+P_{\text {clip }}+P_{\text {quant }_{c}}}
$$

From Figure 2.15, it can be seen that for a given signal power, thermal noise, and quantization noise, there is an optimal crest factor that will result in the highest possible SNR. A low crest factor, meaning that there is a considerable portion of the signal being clipped, will result in the destruction of the desired signal in addition to thermal noise dominating. A high crest factor implies that a major portion of the useful signal desired is condensed within a small amplitude, where both thermal noise and quantization noise will start to have an increasing effect. This will be later confirmed with measurements. 


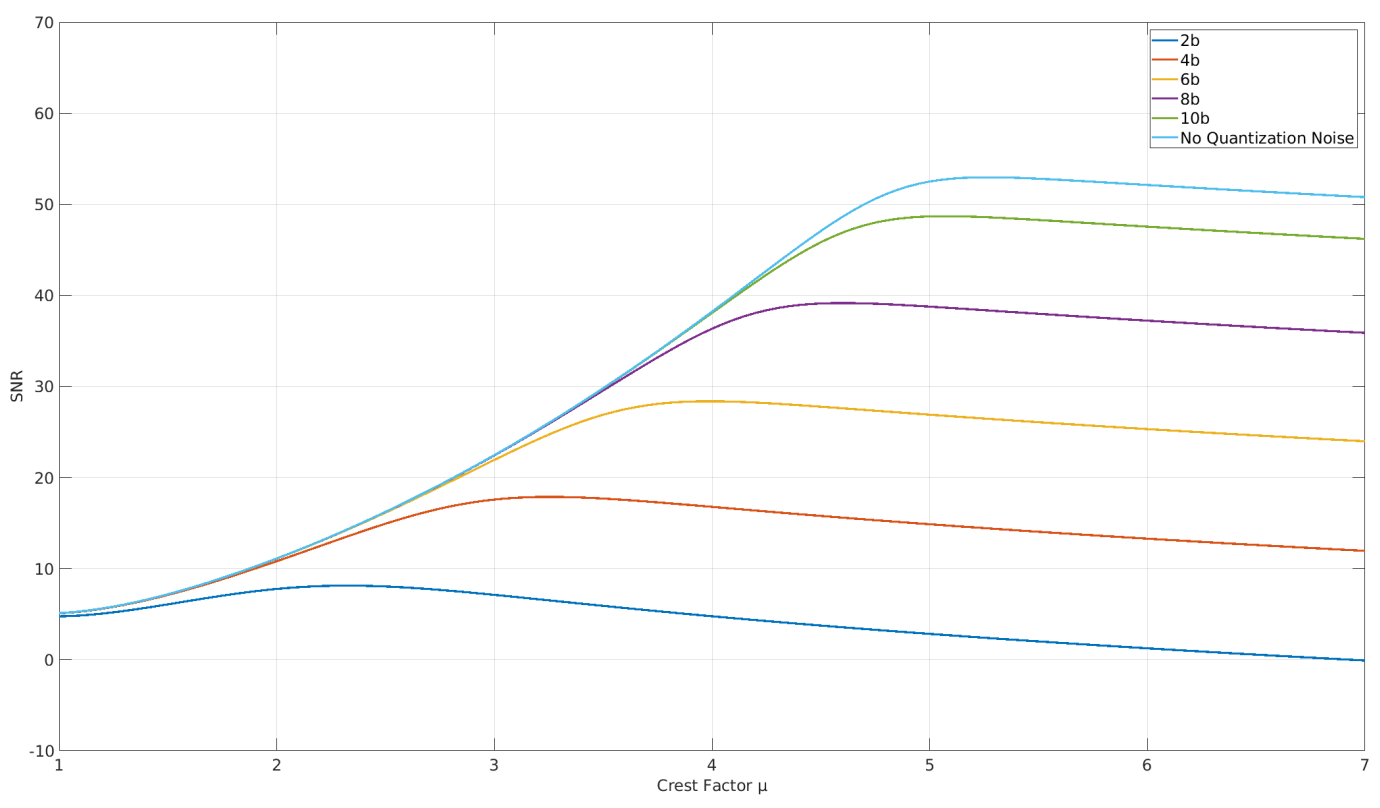

Figure 2.13: SNR Total vs $\mu$

\subsection{Summary}

This chapter presented the fundamentals of two classes of transceivers and modulation techniques. It then introduced the basic concept of this work, DMT, and some of the key characteristics of impairments experienced from an analytical perspective in order to design a system that optimally overcomes them. It concludes with a total SNR equation that takes into account clipping noise, quantization noise, and thermal noise as a function of the clipping (crest) factor. 


\section{Chapter 3}

\section{Design Considerations}

Figures 3.1 and 3.2 demonstrate the implemented DMT transceiver system design, which combines a few optimization techniques that to the knowledge of the author's knowledge have not been used together in such a manner. Namely, these are the combination of using bit-loading, efficient resource utilization by performing dual simultaneous Fourier Transforms, and using a fractional oversampling ratio. In addition to that, a comprehensive analysis and consequence of each design metric and how they impact one another was demonstrated. This analysis includes the investigation of clipping and sub-channel number selection, as they are tightly coupled with the challenge of having a high peak to rms ratio usually encountered with DMT or OFDM. Synchronization between the transmitter and receiver is also demonstrated as it pertains to the specific hardware available in which the system was implemented, and considerations for how it should be implemented for a real world application.

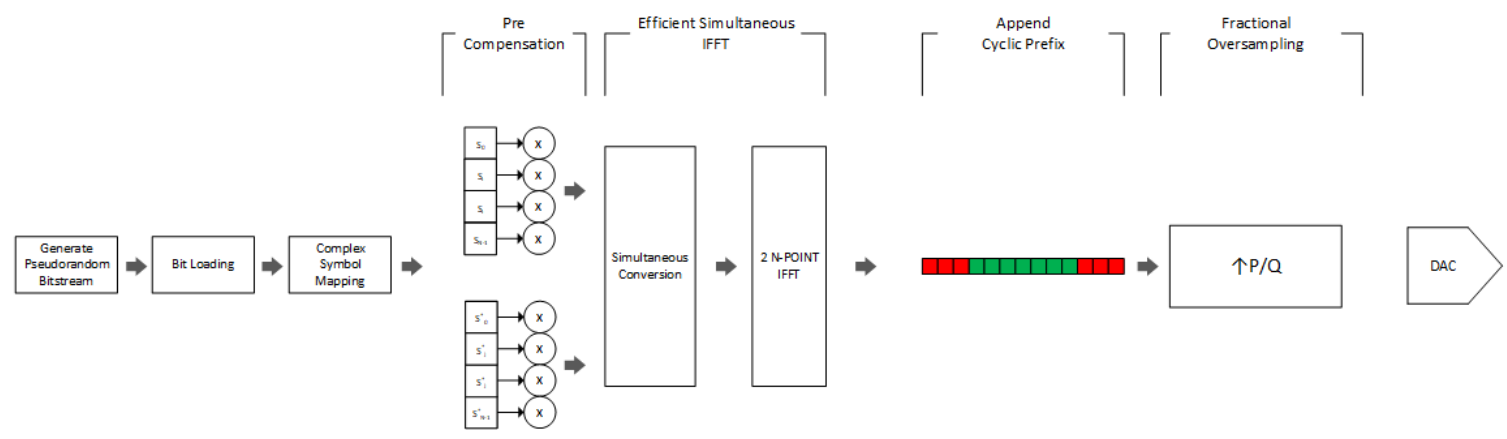

Figure 3.1: DMT Design Tx Block Diagram

From the block diagram perspective, both the optical and electrical implemented systems are identical. The differences arise in the configuration of key parameters. 


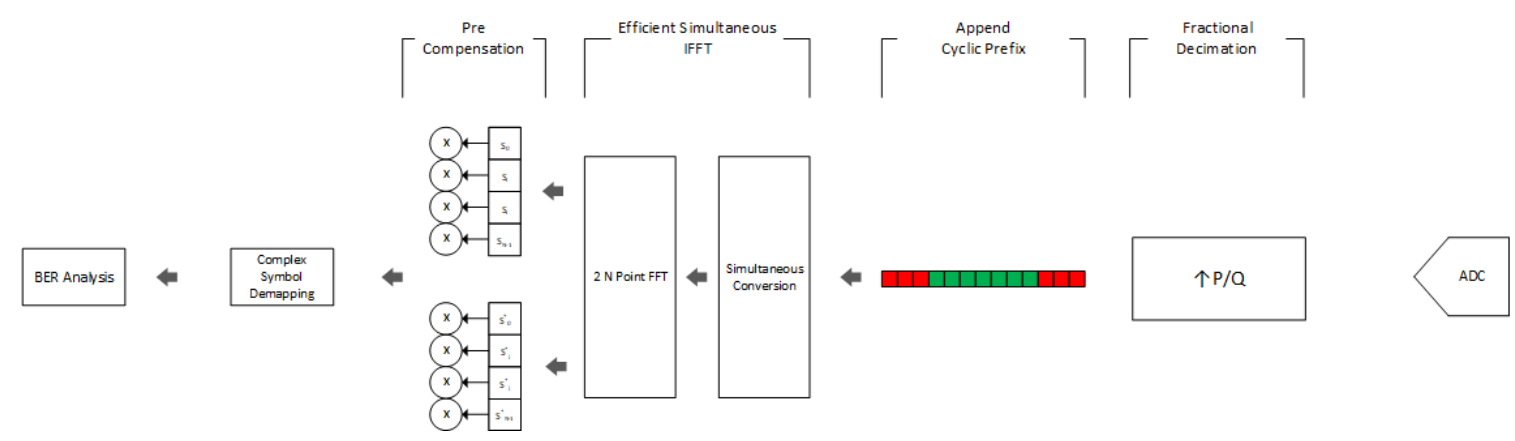

Figure 3.2: DMT Design Tx Block Diagram

These differences arise due to the fact that the hardware available for both systems have different frequency responses (especially with one being an optical channel), non-linearities, jitter, converter sampling frequency, and converter resolution.

\subsection{Channel Number Selection}

The selection of the number of channels will impact a few major performance metrics of a DMT system. A key impairment that DMT is well-suited to overcome is the nonflat frequency response - this is done through having sufficiently narrow channels so that each channels experiences a flat frequency response. However, as the number of channels increases, so does the probability that the PAPR will exceed some threshold $P A P R_{t h}$. To illustrate this, data sets for various channel number configuration were generated in order to experimentally show the increase in the probability of achieving a certain PAPR as the number of channels is increased. It can be seen in Figure 3.3 that for high PAPR, the probability in exceeding that ratio is almost three orders of magnitude larger when comparing the lowest and highest sub-channel count. This may impact the achievable SNR as will be explored later in this work. As seen in Figure 2.13, there is an optimal crest factor that maximizes the SNR with quantization, clipping, and thermal noise taken into account. An increase in the PAPR will require a lower clipping amplitude - which will result in a lower achievable SNR. 


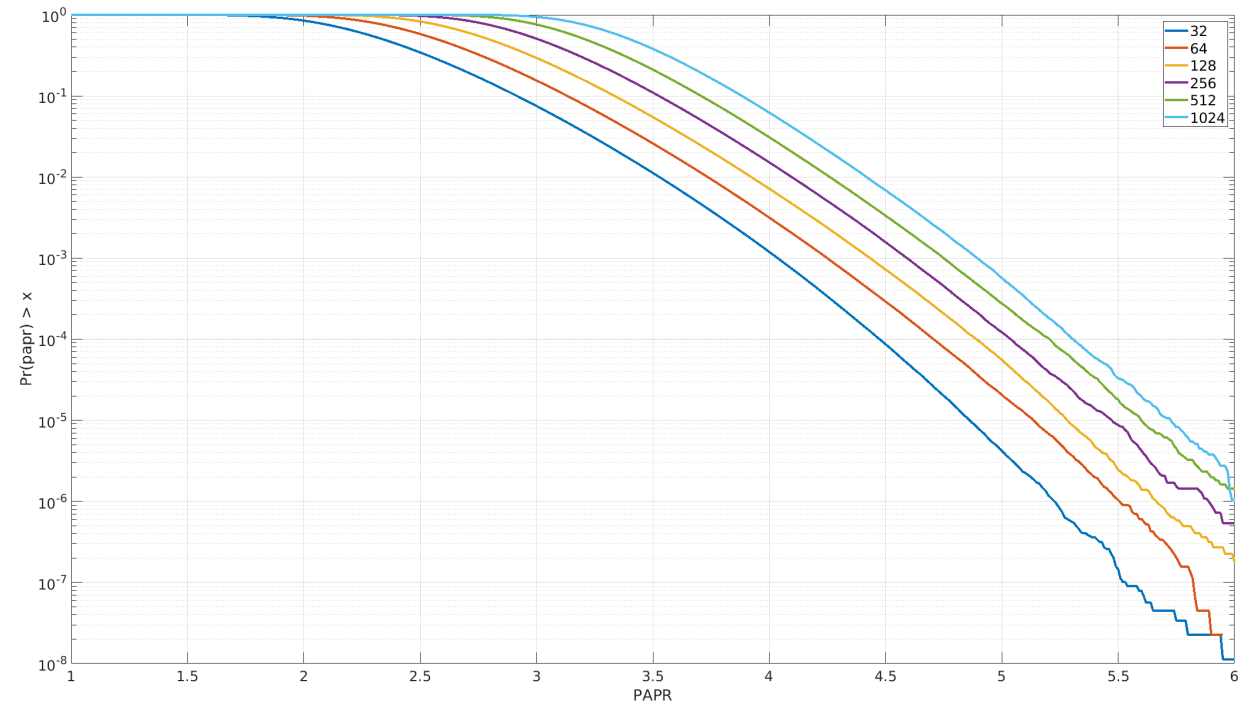

Figure 3.3: Channel Number vs Measured PAPR Cumulative Distribution Function

An analysis of this was done by varying that clipping amplitude while keeping all other parameters fixed, which produces a set of SNR vs $\mu$ for each clipping amplitude using Equation 2.15. Figure 3.4 shows the reduction in achievable SNR as clipping amplitude is decreased to meet the PAPR needed for later stages in the analog chain.

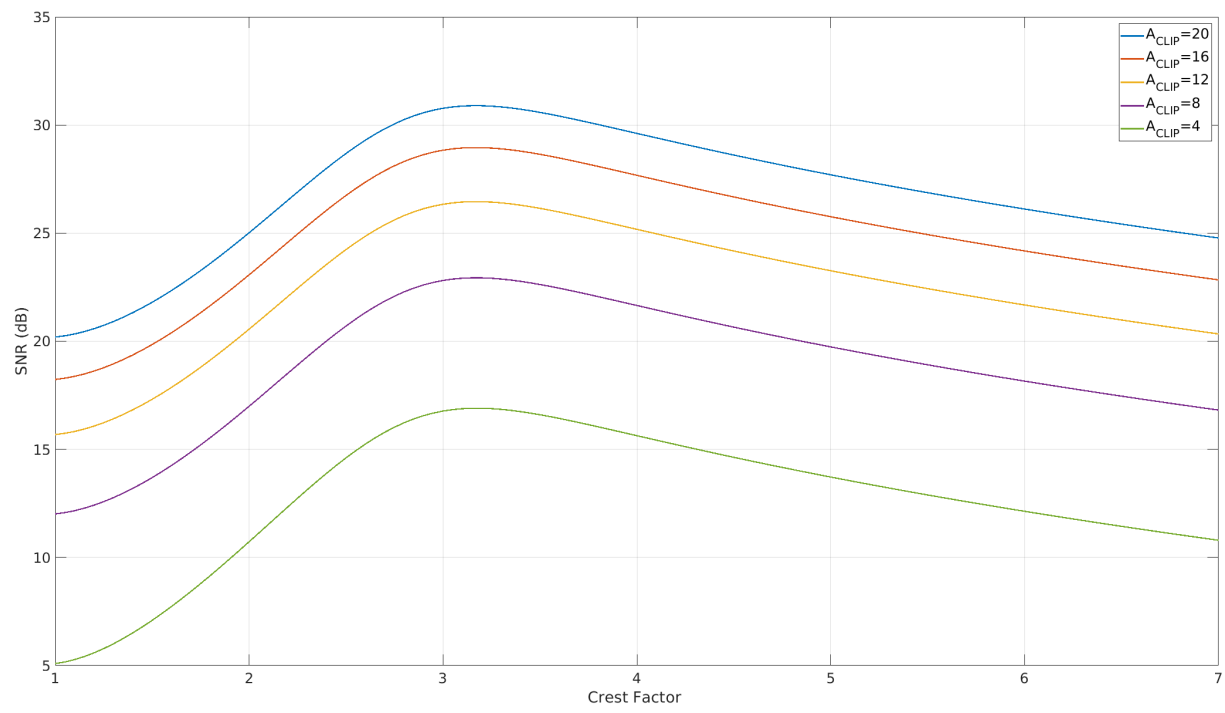

Figure 3.4: SNR vs Crest Factor for different Clipping Amplitude 
Another factor, and likely the most critical in practical applications, is power consumption requirements. The size of the FFT needed to produce the desired number of channels will dramatically increase with the number of channels. Figure 3.5 is derived from [6] whereby a reconfigurable FFT for mobile applications is implemented.

However, this does not taken into account that the required frequency of operation is not constant for different FFT lengths, where they are inversely proportional. Using Equation 3.1, the power consumption for the FFT block was scaled for frequency to obtain Figure 3.6.

$$
P=C \cdot V_{\text {supply }}^{2} \cdot f
$$

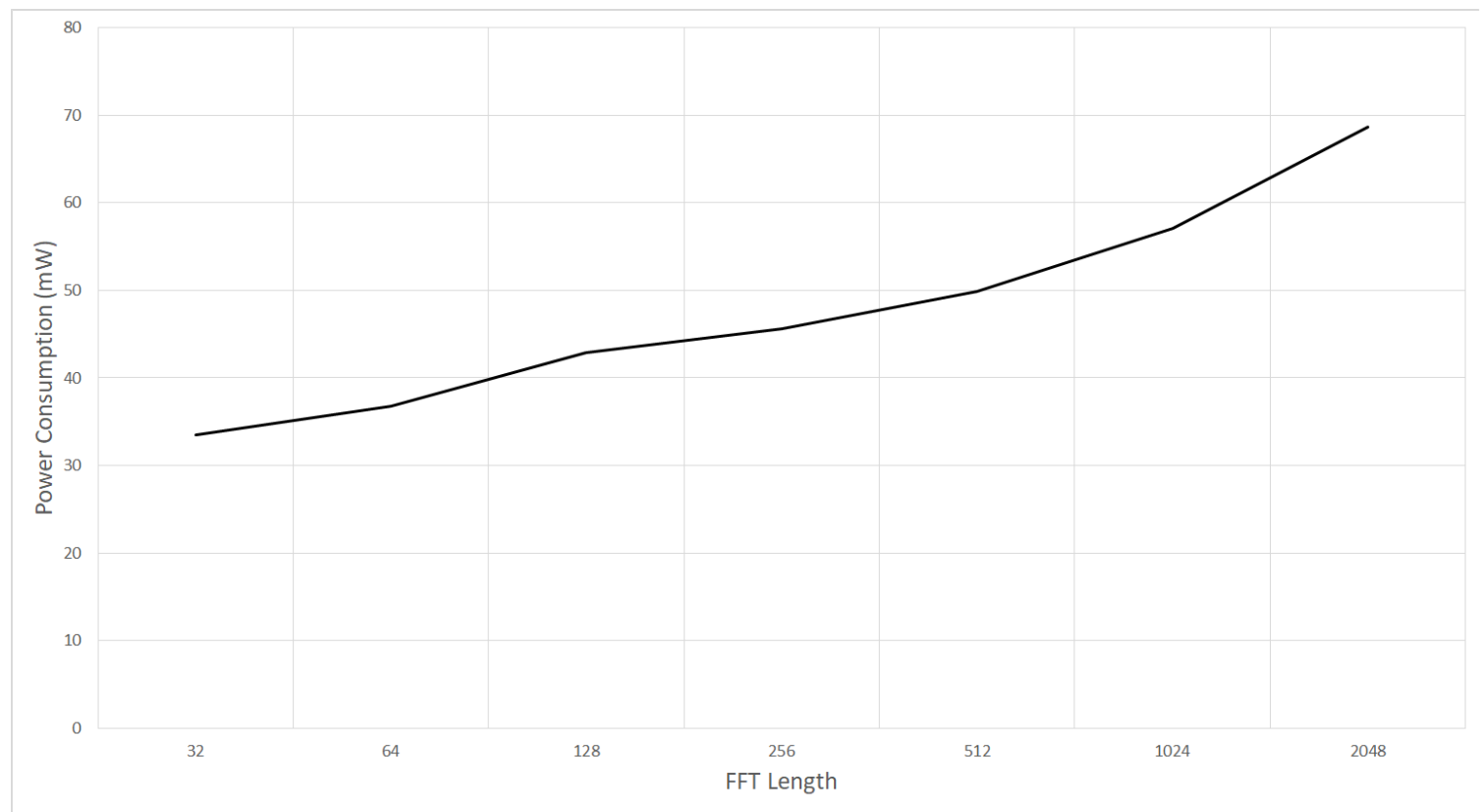

Figure 3.5: Power Consumption of different FFT Length Modes [6] 


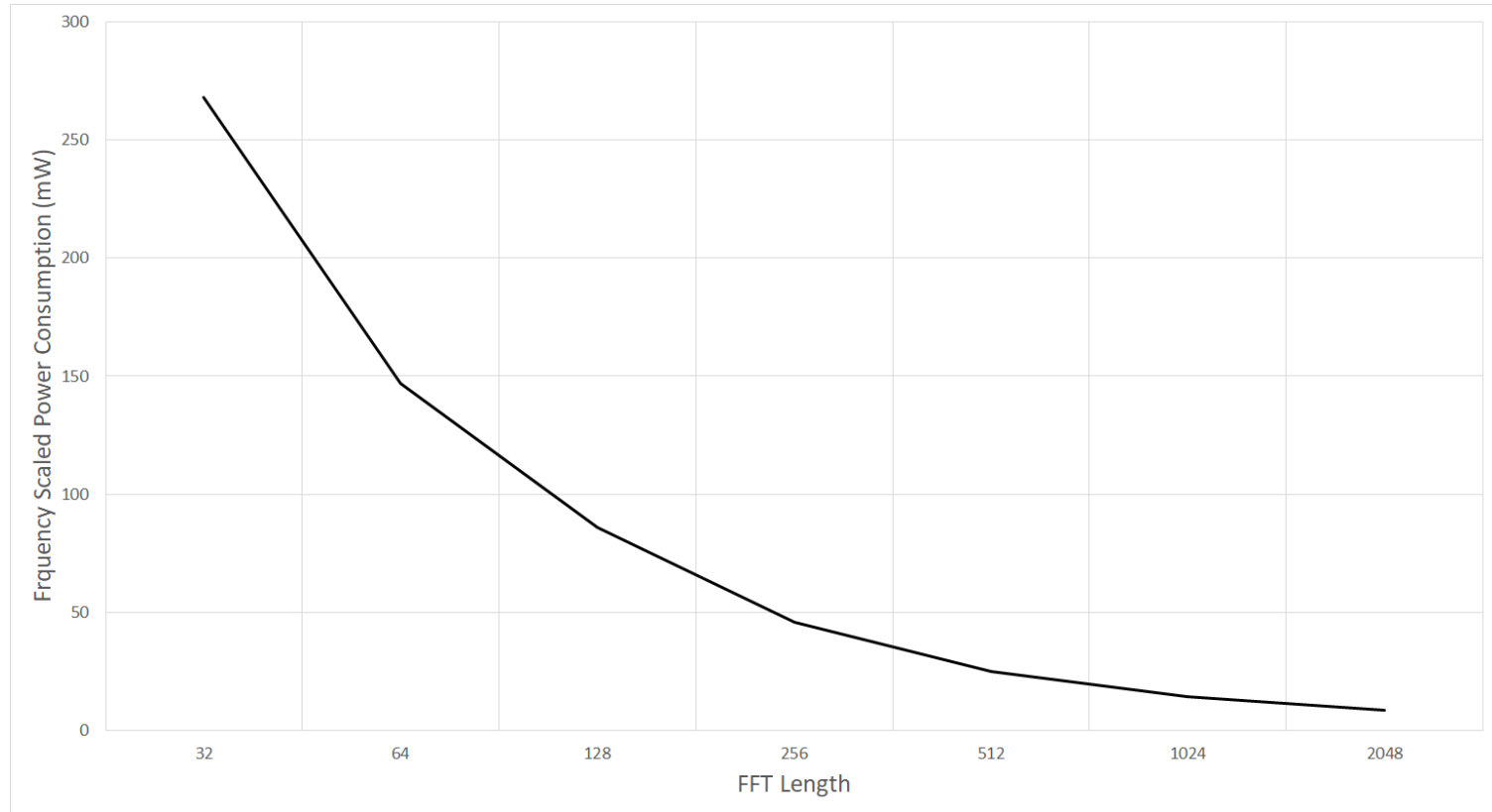

Figure 3.6: Frequency Scaled Power Consumption of different FFT Length Modes

With that into consideration, getting accurate measured values for the power consumption given a frequency of operation will help in the design decisions of FFT size given area and power consumption limitations, as well as generally reduced BER as the FFT length increases.

The increase in PAPR will impact the maximum achievable SNR, as was analysed and demonstrated in section 3.1 despite the usage of an optimal clipping amplitude (or crest factor). However, using a larger number of channels will act to combat the frequency selective response of the channel and the overall system response. This is achieved through having a sufficiently narrow subchannel bandwidth. Measurements were performed for various number of channels to provide an analysis for the effects of channel number selection on the BER of the system and to validate the number of channels used for the final implementation.

Figures 3.7 and 3.8 show the BER for measurements of varying numbers of subchannels, where the number of sub-channels practically has no effect on the BER for the upper end of the spectrum. In this region, the degradation due to attenuation is the dominant effect. For a low number of channels, it can be seen that for a narrow portion of the spectrum, there are large swings in measured BER. This is due to some adjacent channels having a considerable difference in sub-channel SNR, where some subchannels experience a flat response whilst others don't. This will depend entirely 
on the shape of the frequency response.

For a large number of channels, the relatively large swings in BER are eliminated, meaning each sub-channel's frequency response is sufficiently flat. This also provides the benefit of having a larger granularity for using bit-loading more optimally by following the rise in BER with a reduction in bits allocated for the particular subchannel. There is an upper bound on the sub-channel count that will be dictated by the area and latency of the FFT, as well as the increase in PAPR will eventually cause an increase in BER that will be larger than the benefit of having narrow sub-channels.

Due to the low BER and the ability to have a more granular control over the bit-allocation across the spectrum an FFT of length 1024 will be used.

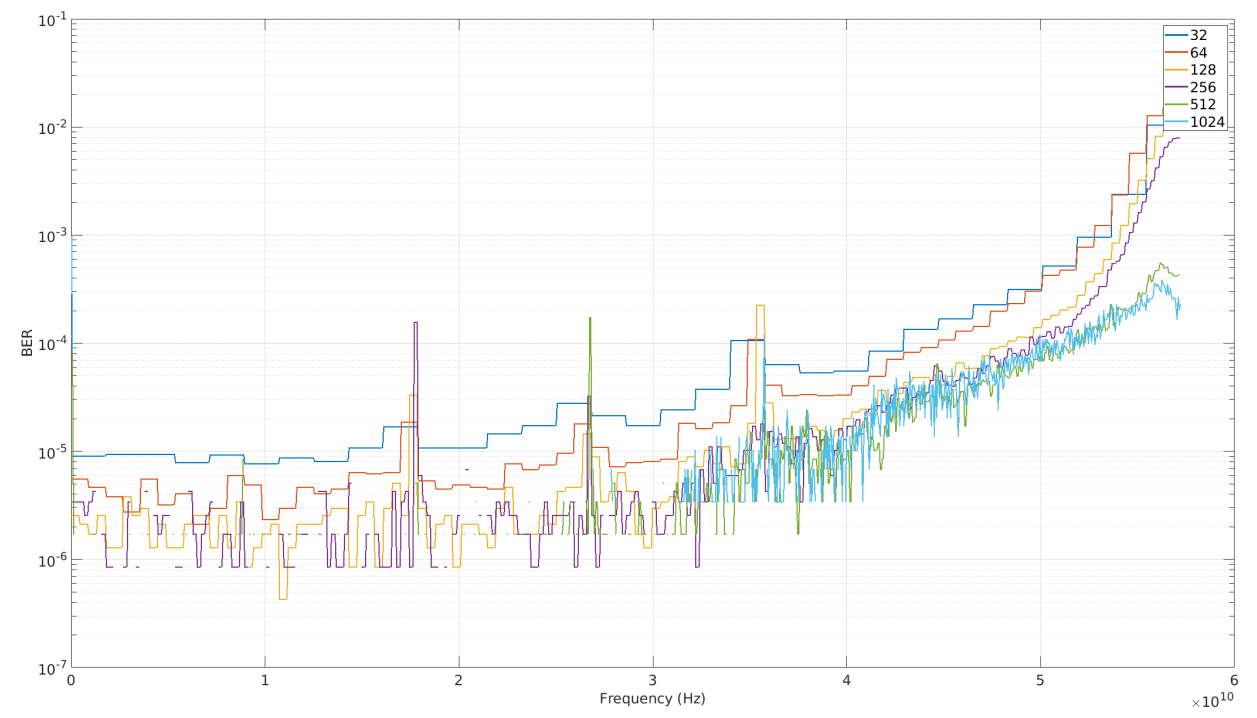

Figure 3.7: BER for Different Sub-Channel Number Schemes (Full Spectrum) 


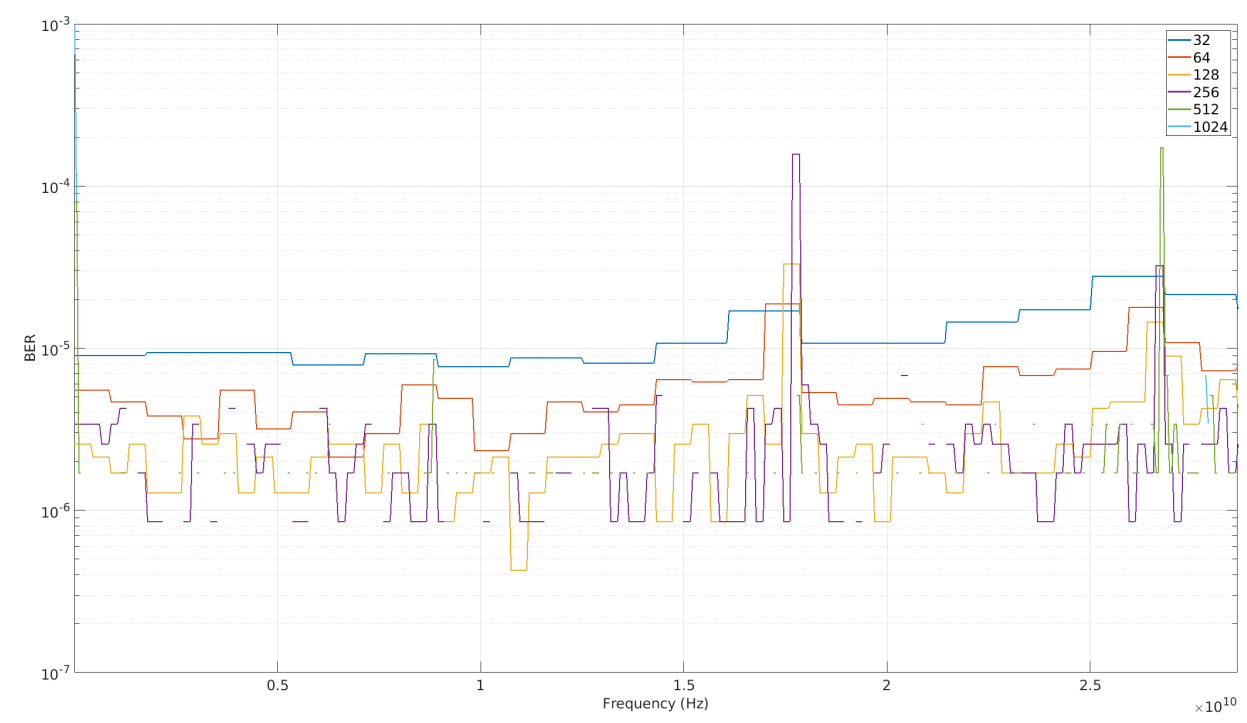

Figure 3.8: BER for Different Sub-Channel Number Schemes (Lower Half Spectrum)

In addition to the points mentioned in this section, an analysis of the constellation distortion is warranted in order to justify two key design considerations. The first being whether pre/post compensation is required, the second being the appropriate number of channels to select. The distortion of the constellation $D_{v}$ is defined as :

$$
D_{v}=\frac{S_{t x}}{S_{r x}}
$$

where $S_{t x}$ and $S_{r x}$ are the complex transmitted and received symbols. $D_{v}$ describes rotation and resizing of the constellation that occurs as a result of all the impairments, scaling, clipping, and attenuation. In other words, it's the complex gain factor that can be applied to the received symbols in order to obtain the originally transmitted set of complex symbols. 


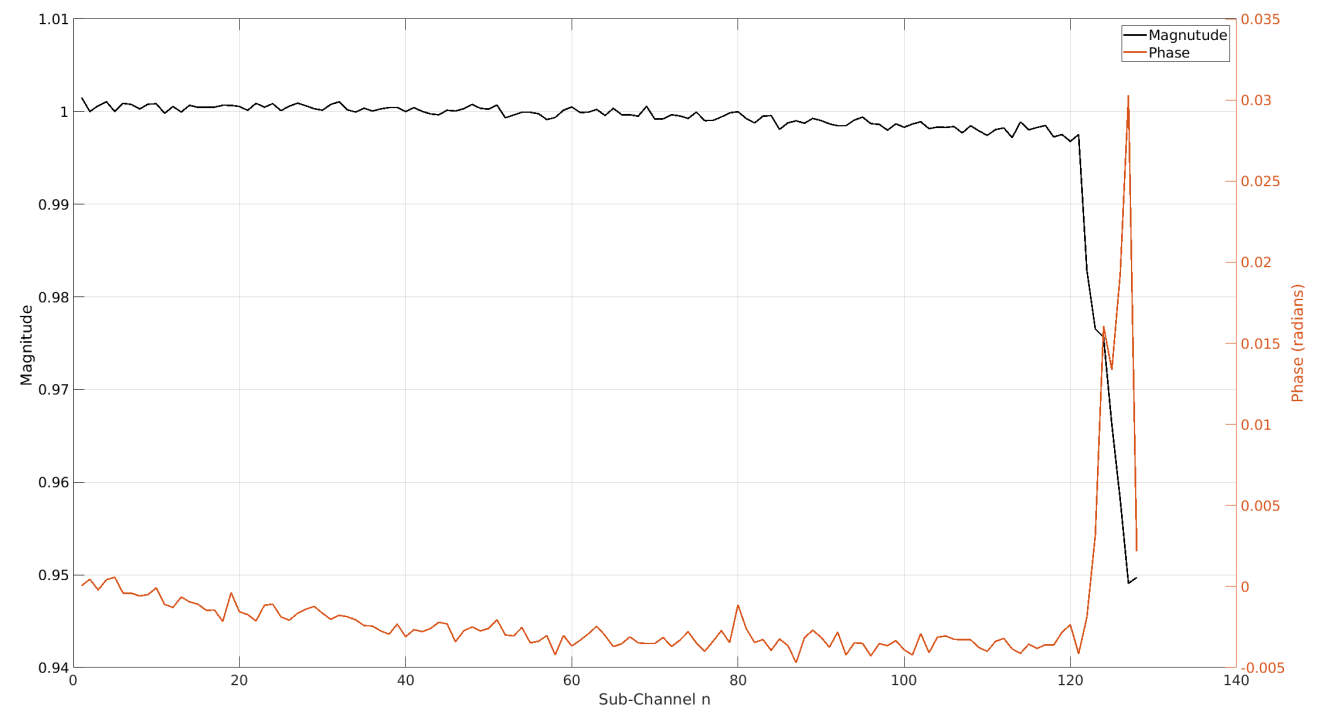

Figure 3.9: Mean Complex Distortion Vector for 128-Channel Scheme

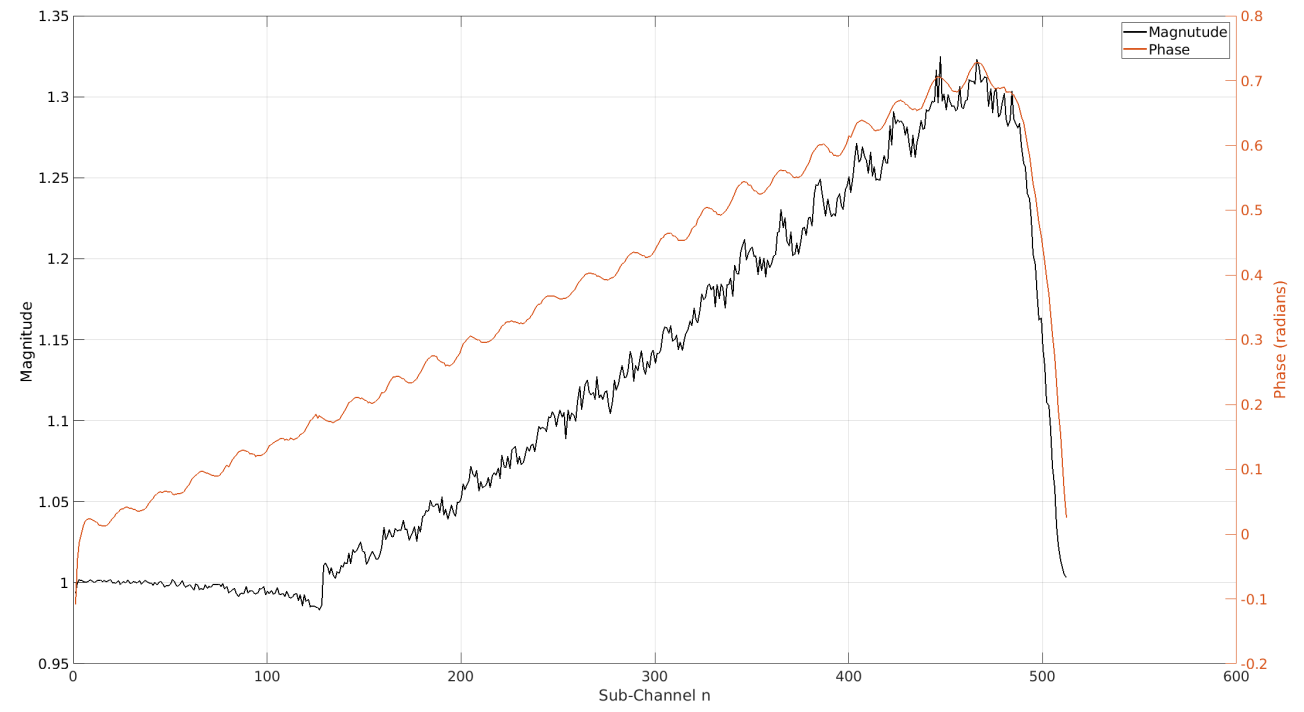

Figure 3.10: Mean Complex Distortion Vector for 512-Channel Scheme

Figures 3.9 and 3.10 further substantiate the advantage of using a higher subchannel scheme. The former demonstrates that frequency response over each subchannel averages out to produce a mean distortion vector with unity magnitude and a zero phase shift, compared with the latter where each sub-channel clearly experiences 
a distinguishable frequency response from its neighbouring sub-channels that can be individually corrected for. A unity mean zero phase distortion vector may give the impression that it is desired, however what this indicates is that sub-channels are experiencing frequency-selective distortion, which goes against the fundamentals of DMT. An extreme case is the use of a signal that spans the entire available bandwidth, such as conventional amplitude or phase modulation. The effect that frequencyselective fading has on the ability to be able to perform pre/post-compensation and subsequent effect on BER will be shown in Section 3.7

\subsection{Oversampling}

Following well known signal processing theory, Nyquist rate is the rate at which a signal can be sampled and reconstructed. Signals sampled below this rate will suffer considerable degradation due to aliasing. This rate is $f_{N}=2 \cdot f_{o}$ where $f_{o}$ is the signal spectrum and $f_{N}$ is the Nyquist sampling rate. One method of increasing the SNR of a sampled signal (by reducing the effect of quantization noise) is to sample a signal at a rate higher than the Nyquist rate. This is known as oversampling, whereby the oversampling ratio is defined as $O S R=\frac{f_{s}}{2 \cdot f_{o}}$. Oversampling in a DMT system will result in a reduction of the throughput equivalent to the oversampling ratio, for the benefit of a reduction in BER. Typically, oversampling ratios are integers. However, this work will explore the usage of fractional oversampling ratios to trade-off the loss in throughput and the bit error rate.

Equation 3.3 indicates that that the overall SNR improves by $6 \mathrm{~dB}$ for every additional bit (n) of resolution to the converter, or by $3 \mathrm{~dB}$ for every doubling of the OSR [26].

$$
S N R \approx 6.02 n+3 \log _{2}(O S R)+1.76
$$

The drastic improvement in BER that seems to contradict what would be expected by a maximum of a $3 \mathrm{~dB}$ SNR increase (due to doubling of OSR) arises from the fact that Equation 3.3 refers to the overall SNR of the signal. However, for DMT, oversampling effectively packs the signal into a region of the spectrum corresponding to the oversampling ratio. Therefore, in addition to reducing quantization noise, sub-channels falling within low SNR regions of the available bandwidth experience a 


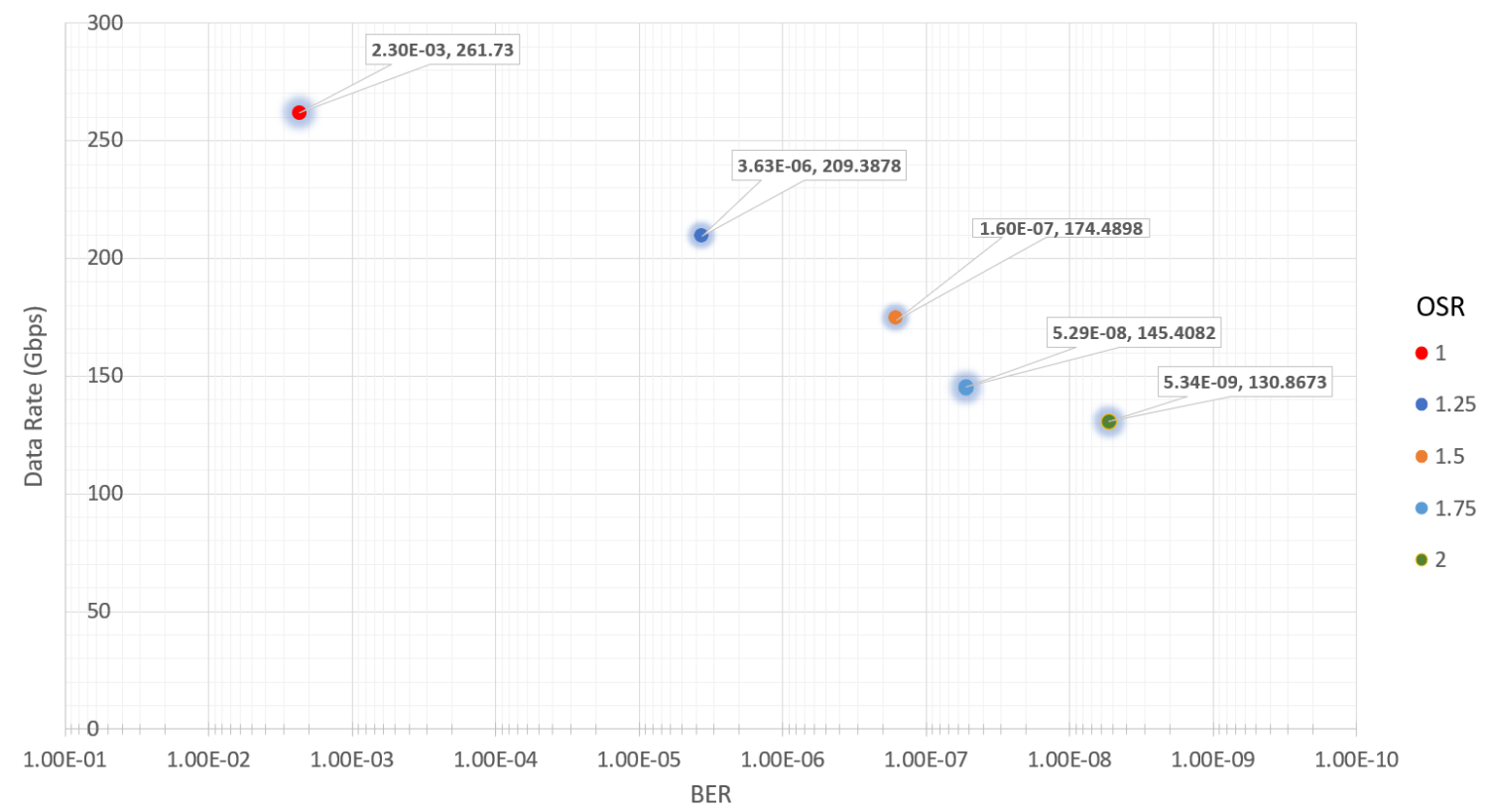

Figure 3.11: Measured Data Rate and BER for Various Fractional Oversampling Ratios

drastic improvement due to being shifted to higher SNR regions.

A fractional oversampling ratio can be achieved through a combination of integer interpolator and decimator in series [27]. An oversampling ratio of a fraction $\mathrm{F}$ can be achieved by using an interpolator of $\mathrm{M}$ and followed by a decimator of $\mathrm{N}$ where $F=\frac{M}{N}$ at the transmitter, and by using a decimator of $\mathrm{M}$ followed by an interpolator of $\mathrm{N}$ at the receiver as shown in Figure 3.12. Typically a decimator is preceded by a bandlimited filter in order to prevent aliasing. Depending on the targeted technology, available area, and power consumption budget, this can be implemented in the digital or analog realm.

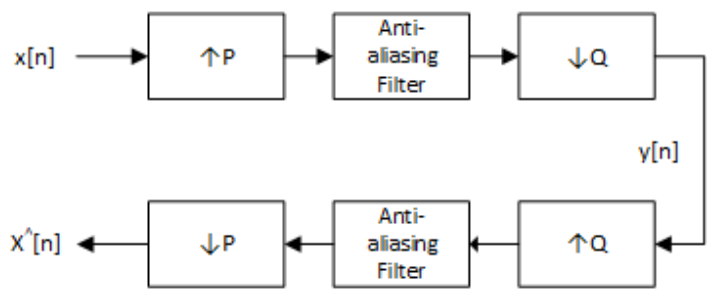

Figure 3.12: Fractional Oversampling Ratio Implementation

The details of this go beyond the purpose of this work. This design takes advantage 
of the multi-rate capabilities of MATLAB to achieve a fractional oversampling ratio.

For this work, an oversampling ratio $\frac{5}{4}$ is selected due to the drastic improvement in bit error with a minimal throughput loss, as can be seen in Figure 3.11. There is almost a three order of magnitude decrease in bit error when going from no oversampling to an oversampling ratio of $\frac{5}{4}$, resulting in an average BER of $3.63 \times 10^{-6}$, making it a great candidate for maximizing throughput through the use of bit loading while maintaining a maximum BER of $3.8 \times 10^{-3}$ that correspond to a HD-FEC (Hard Decision Forward Error Correction) threshold. The improvements in BER are less significant with the increased OSR as there are various other impairments impacting that performance than simply the SNR at the output of the converters.

\subsection{Crest Factor and Clipping}

Measurements were performed with the same setup outlined in Section 4.1 with varying crest factors to compare the analytically derived optimal crest factor for validation. The PAPR of the DMT symbol (output of the IFFT) is measured, and a clipping amplitude is applied to obtain a certain PAPR (or crest factor) after clipping. For the purpose of validation, the BER was measured for the first quarter of the spectrum to eliminate outliers from overshadowing the performance boost in the case of a flat modulation (ie. no bit-loading). The number of channels used is 256 , with a $\mathrm{CP}$ length of about 18\%. From Figure 3.13 the optimal crest factor is around 3. Finer measurements performed in that trough region showed an optimal crest factor of 2.85 . On initial inspection, this coincides with the theoretically trend shown in 2.13 in that the BER (direct effect of SNR) is drastically low at low crest factors. It then rises and peaks before slowly declining due to trading off clipping noise to quantization noise. In addition to that, higher crest factors will cause higher degradation due to nonlinearities in the analog chain (which are not measurable for the purpose of this work due to the available hardware). One can conclude from this that clipping is needed to improve system performance, as no clipping, which can be approximated as a high crest factor (refer to Equation 2.6), shows over an order of magnitude in the increase of the BER. 


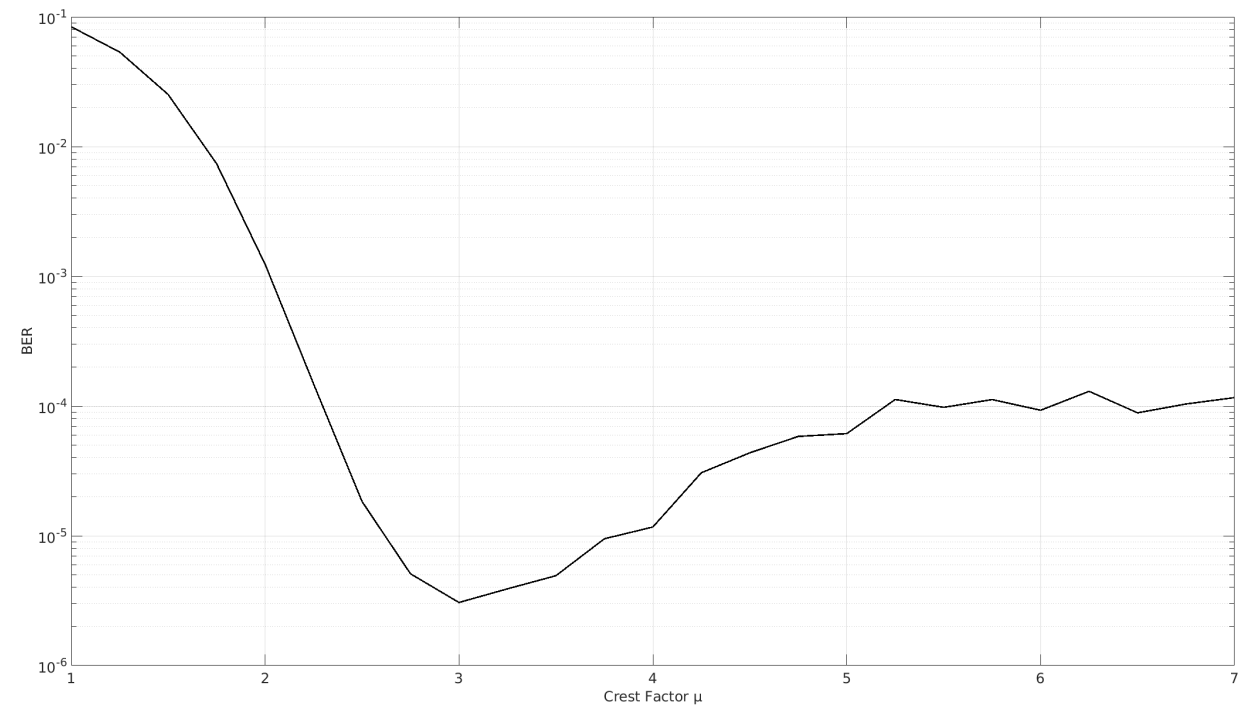

Figure 3.13: BER vs Crest Factor

\subsection{Bit-Loading}

With the advantage of highly configurable DSPs, one can maximize the overall throughput of a DMT system while maintaining a target BER by using bit-loading. This technique allows for a variable number of bits assigned per symbol for each channel depending on the SNR of the respective channel. Formally, from [28] the concept is to :

$$
\text { Maximize } \sum_{i=1}^{N} b_{i} \text { under the constraint of } \frac{\sum_{i=1}^{N} b_{i} P_{i}}{\sum_{i=1}^{N} b_{i}} \leq P_{T}
$$

where $b_{i}$ and $P_{i}$ are the number of bits and BER of the i'th channel respectively and $P_{T}$ is the target overall BER.

The bit-loading scheme used for the electrical-channel DMT system implemented in this work can be seen in Figure 3.14. 


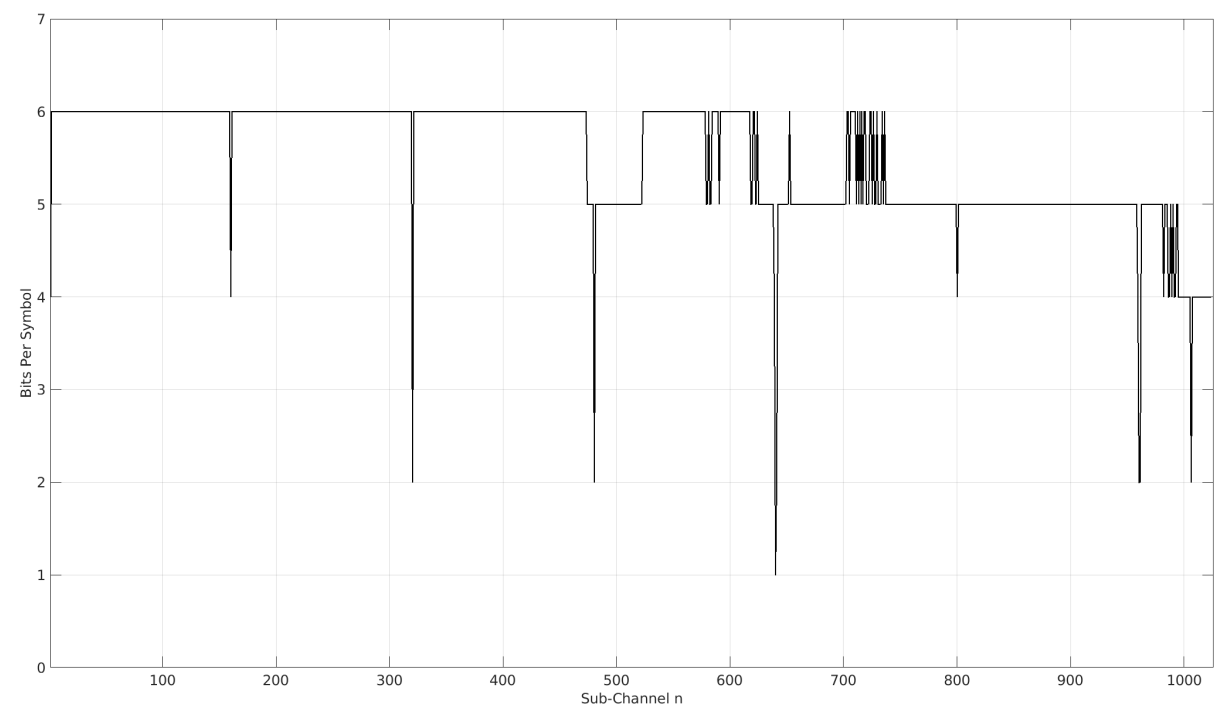

Figure 3.14: Bit Loading

\subsection{Efficient Fourier Transform}

As shown in $[20]$,in order to obtain a purely real DMT frame, the input must satisfy Hermitian symmetry. This results in a FFT length of $2 \cdot N$ where $\mathrm{N}$ is the number of sub-channels, which can be considered as an inefficient use of hardware or computational resources. This can be improved upon by considering the following intrinsic properties of Fourier Transforms.

Appendix A demonstrated that in the forward direction, a complex FFT of length $\mathrm{N}$ in addition to some arithmetic transformation can be used to obtain the FFT of 2 purely real signals.

Given

$$
Y(f)=X_{1}(f)+X_{2}(f)
$$

In order for $x_{1}(t)$ and $x_{2}(t)$ to be respectively purely real and purely imaginary, $X_{1}(f)$ must be an even function, while $X_{2}(f)$ must be an odd function.

Suppose $\mathrm{C}(\mathrm{f})$ and $\mathrm{D}(\mathrm{f})$ are two consecutive frequency domain DMT symbols, where each is of length $\mathrm{N}$. Using the above properties the following can be performed prior to passing these symbols into the IFFT :

$X_{1}(f)$ can be constructed as follows : 


$$
X_{1}(f)= \begin{cases}C(f) & 0 \leq x \leq N-1 \\ C^{*}(f) & N \leq x \leq 2 N-1\end{cases}
$$

While $X_{2}(f)$ can be constructed as follows :

$$
X_{2}(f)= \begin{cases}D(f) & 0 \leq x \leq N-1 \\ -D^{*}(f) & N \leq x \leq 2 N-1\end{cases}
$$

From [29], the simultaneous IFFT of $X_{1}(f)$ and $X_{2}(f)$ can be performed as follows. The real and imaginary portions of $\mathrm{Y}$ can be computed in terms of the real and imaginary components of $X_{1}(f)$ and $X_{2}(f)$ :

For $\mathrm{k}=0, \ldots \mathrm{N}-1$

$$
\begin{aligned}
& Y_{r}(f)=X_{1 r}(f)-X_{2 r}(f) \\
& Y_{i}(f)=X_{1 r}(f)+X_{1 r}(f)
\end{aligned}
$$

Taking the IDFT of $\mathrm{Y}(\mathrm{n})$ to obtain $\mathrm{y}(\mathrm{n})$

$$
\begin{aligned}
& x_{1}(n)=y_{r}(n) \\
& x_{2}(n)=y_{i}(n)
\end{aligned}
$$

where $x_{1}(n)$ and $x_{2}(n)$ are two time domain DMT symbols corresponding to $\mathrm{C}(\mathrm{f})$ and $\mathrm{D}(\mathrm{f})$ respectively, which have been obtained using a single FFT of length $2 \mathrm{~N}$. This is compared to what's typically done where an FFT of length $2 \mathrm{~N}$ is needed for one DMT symbol of length N.

\subsection{Synchronization}

As previously mentioned, the system implemented in this work for the electrical channel case is considered a non-coherent one, meaning it does not contain a CDR. However, there is the need to maintain some alignment between the transmitter and the receiver. Two points will be discussed here, the first applies to what was performed in the context of the system implemented, the other point will address what would need to be performed on a real-world scenario whereby the limitation of the hardware 
at hand are not a concern.

Due to the fact that the transmitter and receiver both have a memory and interface to the DSP, where a snapshot of the entire sequence is continuously being read from memory and placed into the DAC, and the ADC is continuously sampling and storing this snapshot in memory, the data is shifted circularly some unknown number of discrete samples. In order to rotate the received waveform back to align with the transmitted signal before demodulation, a cross-correlation is performed to obtain this discrete skew. The cross correlation is mathematically described as follows :

$$
r_{x}(t) \otimes t_{x}(t)=\int_{-\infty}^{+\infty} r_{x}^{*}(\tau) t_{x}(\tau+t) d \tau
$$

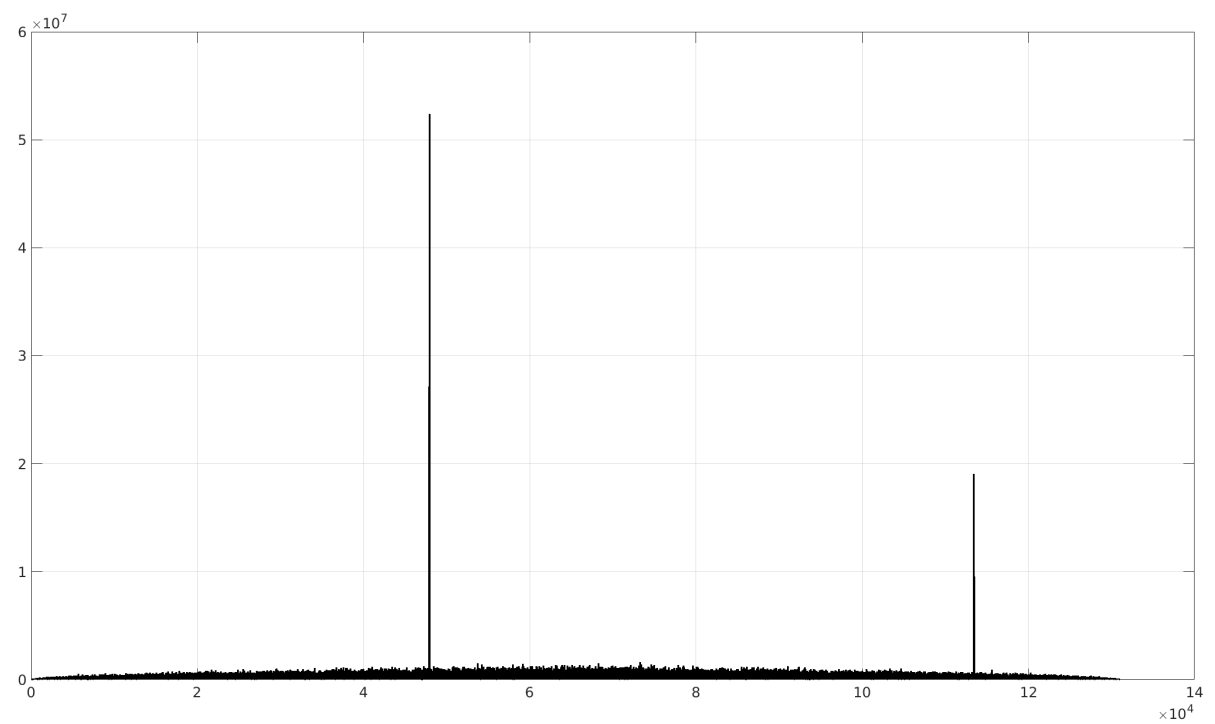

Figure 3.15: Tx and Rx Cross-Correlation

It should be noted that this does not mitigate the jitter between the clocking mechanisms of the ADC and DAC, which would represent what would occur in a real-world application. Therefore, the rms jitter of the ADC and DAC individually would need to be reduced as much as possible to prevent performance degradation.

In a real-world application, the constant deviation of the sampling clock between the transmitter's (DAC) clock $c_{t} x$ and receiver's (ADC) clock $c_{r} x$ need to be corrected for. The deviation is represented as follows : 


$$
\begin{gathered}
c_{t x}=A \sin \left[2 \pi f_{s} t+\phi_{t x}(t)+\theta_{t x}\right] \\
c_{r x}=A \sin \left[2 \pi f_{s} t+\phi_{r x}(t)+\theta_{r x}\right]
\end{gathered}
$$

Here, $f_{s}$ is the sampling clock, $\phi(t)$ is random fluctuations (or jitter), and the fixed delta deviation between the transmitter and receiver is represented by $\left|\theta_{t x}-\theta_{t x}\right|$

Eliminating this deviation can be done through the use of a pilot tone sequencing and provisioning procedure. In addition to evaluating the SNR for each sub-channel, the receiver can cycle through increments of adjustable phase offset to the clock, and select the one that minimizes the BER at the receiver. During this procedure, a set of pre-known symbols can be continuously transmitted until the receiver has adjusted its parameters.

\subsection{Pre and Post Compensation}

For a DMT system, compensating for deterministic impairments and channel loss can be performed in the frequency domain relatively easily due to the readily available frequency bound symbols in the digital domain through the use of basic complex arithmetic. For this work, pre-compensation is defined as the shaping of the constellation applied at the transmitter prior to the IFFT operation. Post-compensation is defined as that same operation, however it is done at the receiver side, proceeding the FFT operation.

It was shown in Figure 3.9, that the width of a sub-channel results in a mean distortion vector of magnitude 1 and 0 phase. This is due to the averaging out of the frequency response over that frequency range. It is therefore predicted that pre-compensating (or post-compensating) will not improve the BER of the system. This was substantiated as can be seen in the measured BER in Figures 3.16 and 3.17 ; in fact the BER was higher when using pre-compensation (in addition to the required post-compensation due to clipping and scaling) versus without it. The cause for the degradation is likely mainly due to the increase in PAPR when using

pre-compensation. As seen earlier in this work, an increased PAPR will require a clipping amplitude to be used in order to achieve the optimal crest factor, causing 


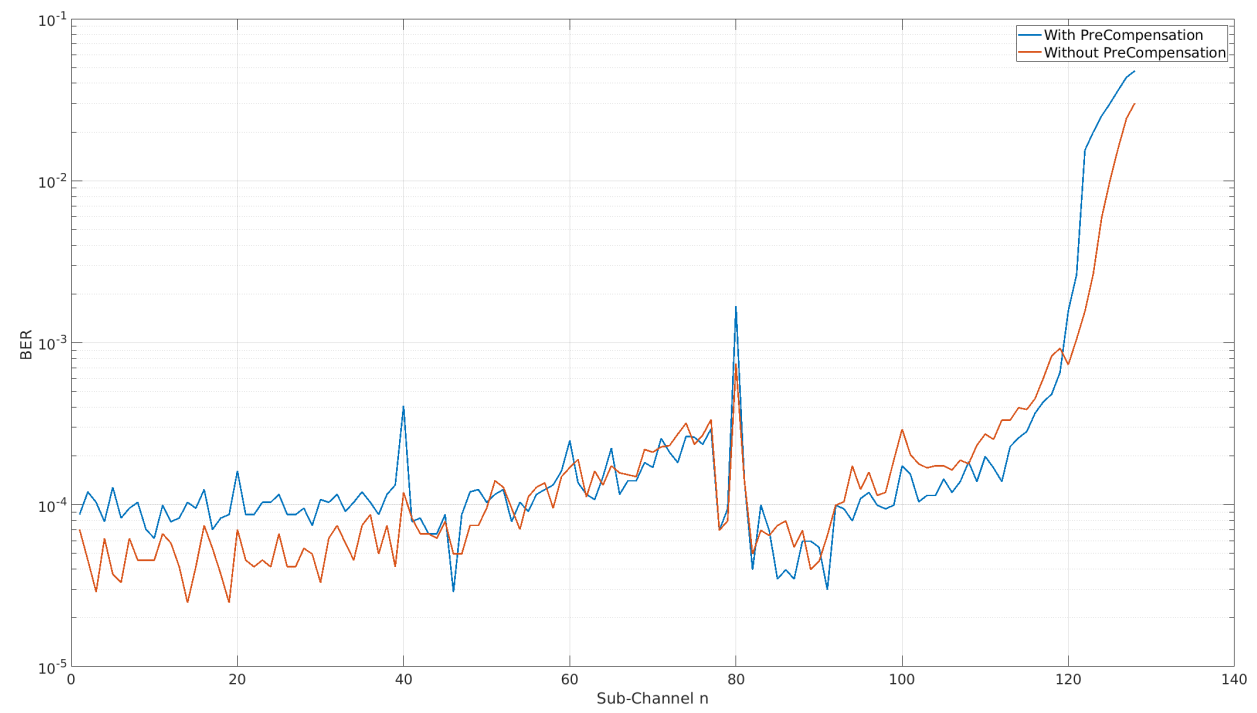

Figure 3.16: Comparing BER for Precompensated vs Non-Precompensated for 128 Sub-Channels Scheme

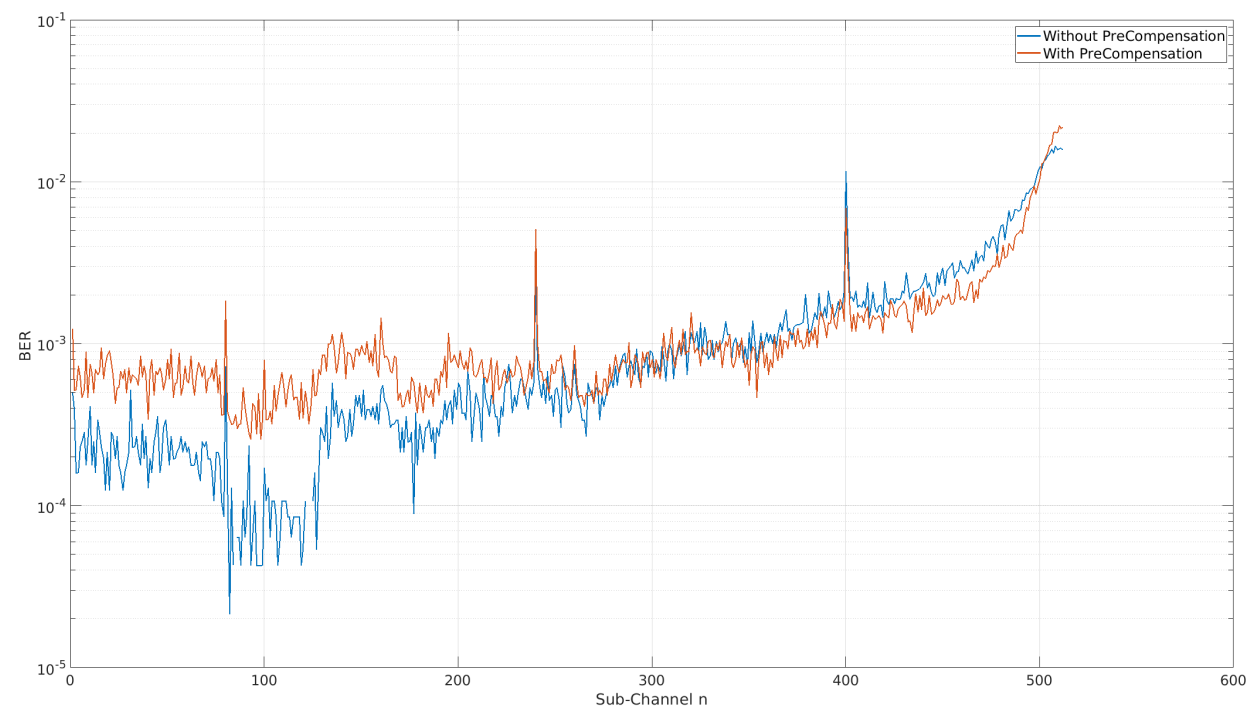

Figure 3.17: Comparing BER for Precompensated vs Non Pre-compensated for 512 Sub-Channels Scheme 
a reduced SNR. This can be seen in Figure 3.4. The analytical analysis of why the PAPR is higher for pre-compensated signals is beyond the intentions of this work. This leads to the possibility of using post-compensation. Since this occurs at the end of the receiver chain in the digital domain, where linearity is of no concern due to the signal being in the digital domain, it may offer advantages over pre-compensation.

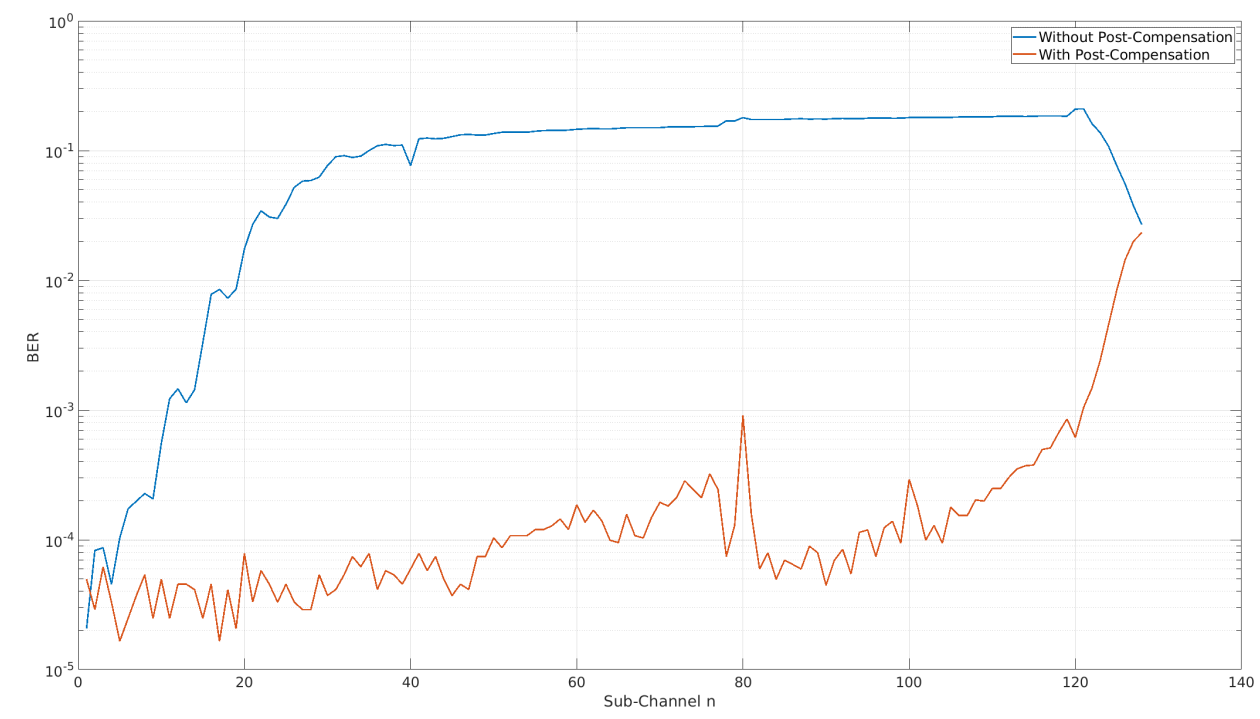

Figure 3.18: Comparing BER for Post compensated vs Non Post-compensated for 128 Sub-Channels Scheme

Post-compensation is performed by first collecting the average distortion vector given known set of predetermined transmit symbols. Firstly, this takes care of any rotation and scaling of individual sub-channels, which conforms to one of the main advantages of using DMT. Figures 3.18 and 3.19 demonstrate the necessity of postcompensation and its advantage over pre-compensation as there are no implications on non-linear effects of high PAPR and clipping that pre-compensation has. One can also notice in the case of Figure 3.19, where 512 sub-channels are used, that there is a more gradual increase in BER as one moves higher in frequency which is substantiated by Figure 3.10 whereby there is more deterministic distortion in the individual sub-channel constellations. 


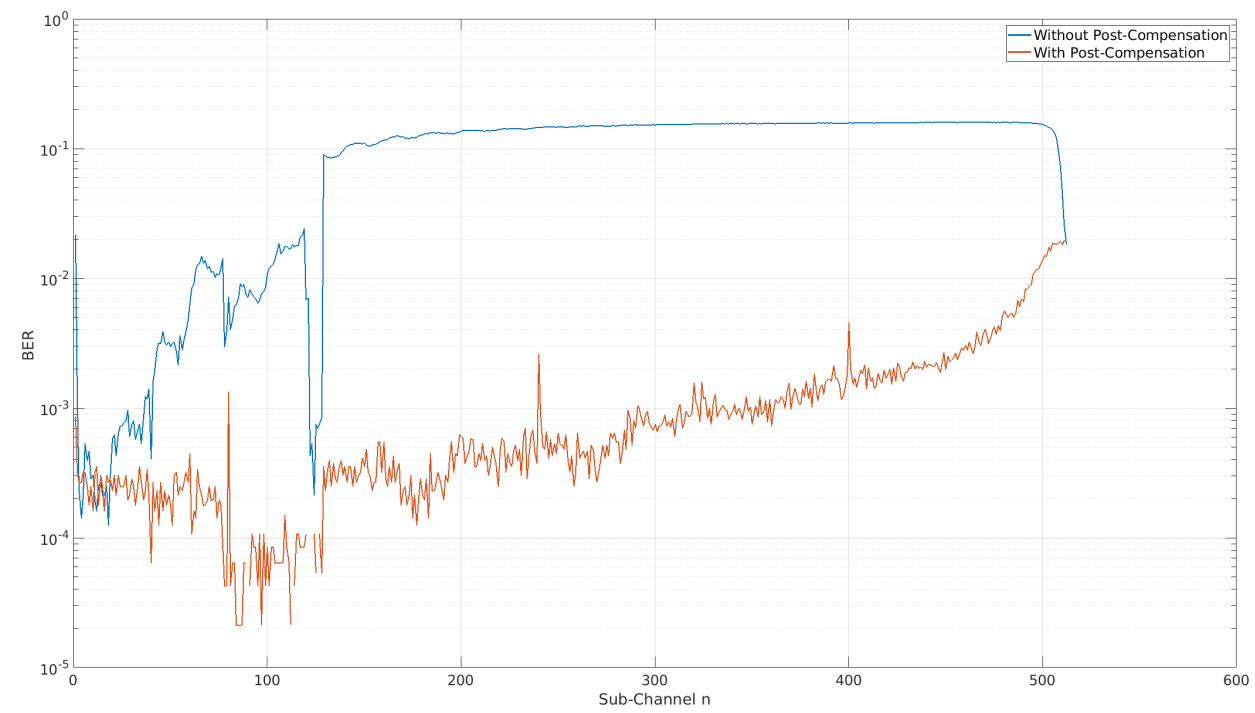

Figure 3.19: Comparing BER for Post compensated vs Non Post-compensated for 512 Sub-Channels Scheme

\subsection{Summary}

This chapter presented the design strategy and techniques used to develop a DMT system that provides optimal desired performance for throughput and bit error rate within a given set of constraints such as analog hardware available, transmit power, and bandwidth of the system. As will be shown in the next chapter, the given constraint was the hardware available where the DMT system was implemented. In addition to that, novel optimization techniques was shown to have significant impact on bit error rate. Finally, a set of key characteristics of the system were summarized in Table 3.1 for both types of systems implemented. It's noted that the optical system was not fully optimized due to time limitation, limited access to equipment, and discoveries made through the electrical system measurements that were achieved later into the project. 
Table 3.1: Summary of Design Points for Implemented DMT Systems (Electrical Channel and Optical Channel)

\begin{tabular}{|l|l|l|}
\hline Design Point & Optical DMT & Electrical DMT \\
\hline IFFT/FFT Length & 128 & 1024 \\
\hline Cyclic Prefix Length & $1.2 \%$ & $0 \%$ \\
\hline Crest Factor & 4.5 & 2.85 \\
\hline Modulation Scheme & $32 / 16 / 4 \mathrm{QAM}$ & $64 / 32 / 16 / 4 \mathrm{QAM}$ \\
\hline Oversampling Ratio & 1.25 & 1.25 \\
\hline DAC/ADC Sampling Frequency & $68.16 \mathrm{GS} / \mathrm{s}$ & $100+\mathrm{GS} / \mathrm{s}$ \\
\hline Full Bandwidth & $34.08 \mathrm{GHz}$ & $42.94 \mathrm{GHz}$ \\
\hline Sub-Channel Width & $233.25 \mathrm{MHz}$ & $42 \mathrm{MHz}$ \\
\hline
\end{tabular}




\section{Chapter 4}

\section{Measurements \& Results}

This chapter will outline the implemented DMT system in both an electrical channel and an optical channel. A DMT system comprised of a combination of softwarebased DSP in addition to existing hardware was constructed for both an electrical and an optical channel. For any DSP needed, MATLAB was the tool of choice, as a framework was built around it that allows directly injecting data into the hardware used to perform measurements. This chapter concludes with a power consumption estimate.

For both cases, electrical and optical, the signal pre \& post processing is thoroughly discussed and justified. The overall system frequency response is also presented to shed light on the capabilities of DMT in high-loss channels. In addition to that, the specifications of the DACs and ADCs are shown as they have a critical impact on the performance of the system and are considered to be the bottle neck in DMT based communication. The result and performance of both cases is summarized.

There are two main distinctions between the two cases explored. Firstly, an optical channel has additional impairments to electrical channels including chromatic, modal dispersion (in multi-mode waveguides), and polarization error. Secondly, the optical channel case is considered to be a coherent system as there is clock (carrier) recovery performed at the receiver. This is contrary to the electrical channel where there is no coherence between the transmitter and receiver. Another difference is that the electrical channel will generally have a larger attenuation with higher frequencies. 


\subsection{Electrical System}

\subsubsection{System Description}

An existing evaluation-board containing a test chip was utilized for the electrical channel measurements of the DMT system. The chip houses the integrated ADC and DAC. DMT symbols are generated in MATLAB and uploaded to the transmit memory of the circuit. The received DMT symbols are extracted from the receive memory and post-processed in MATLAB. Due to the limitation of the system, in particular the size of the internal memories and the inability to continuously place data on the ADC and extract it from the DAC, multiple iterations of uploads and downloads are needed. This was done through an IP interface between MATLAB and the evaluation-board. The transmitter and receiver are connected in loop-back using an interposer board as can be seen in Figure 4.1.

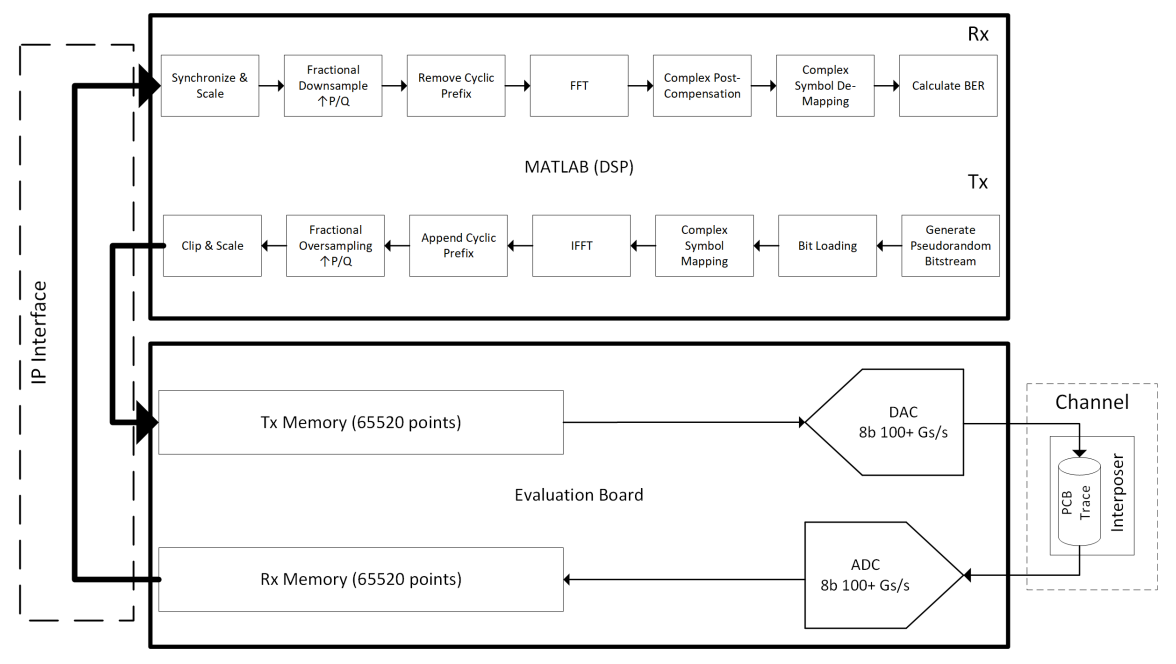

Figure 4.1: Block Diagram of Electrical System Measurement Setup

Figures 4.3 and 4.4 show the overall frequency response of the system which includes the entire chain from DAC to ADC (which includes the channel comprised of the interposer board and the $2 \mathrm{~cm}$ PCB trace), where the loss at Nyquist rate is approximately 18dB. This places this system at the edge between a VSR and MR channel, as categorized in Figure 2.4. There were no additional impairments injected onto the signal such as noise as there are already sufficient impairments present, including thermal noise, jitter of data converter clocks, non-linearities in the system, 


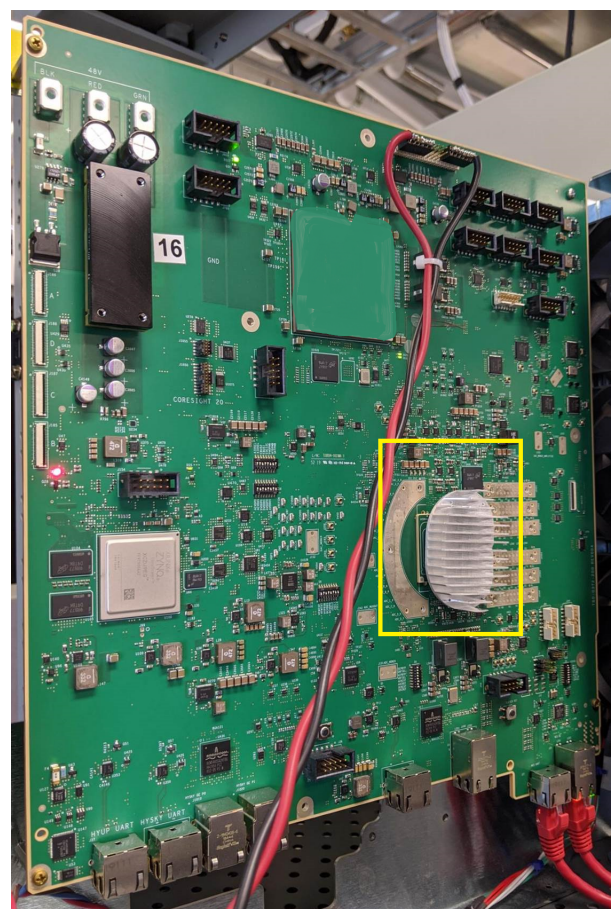

Figure 4.2: Evaluation Board indicating ADC/DAC Integrated Circuit and Interposer (yellow box)

and limited bandwidth and resolution of data converters.

\subsubsection{Transmitter}

A pseudo-random generator is used to generate bits that are parallelized into $\mathrm{N}$ channels and mapped onto a complex point on the IQ plane depending on the modulation order of the respective channel. The bit loading scheme is selected such that the an upper limit of $3.8 \times 10^{-3}$ BER is achieved across all subchannels, corresponding to a HD-FEC limit [30]. A complex factor gain can be applied to the generated constellation before passing it through to the IFFT block. This is typically referred to as pre-compensation.

The output of the IFFT, a time domain DMT symbol, is clipped to achieve a desired crest factor. For the purpose of this experiment, the standard deviation $\mu$ of the entire data set to be transmitted is measured, in order to determine the clipping amplitude $A_{\text {clip }}$ for a desired crest factor $\mu_{\text {desired }}$ as shown below : 


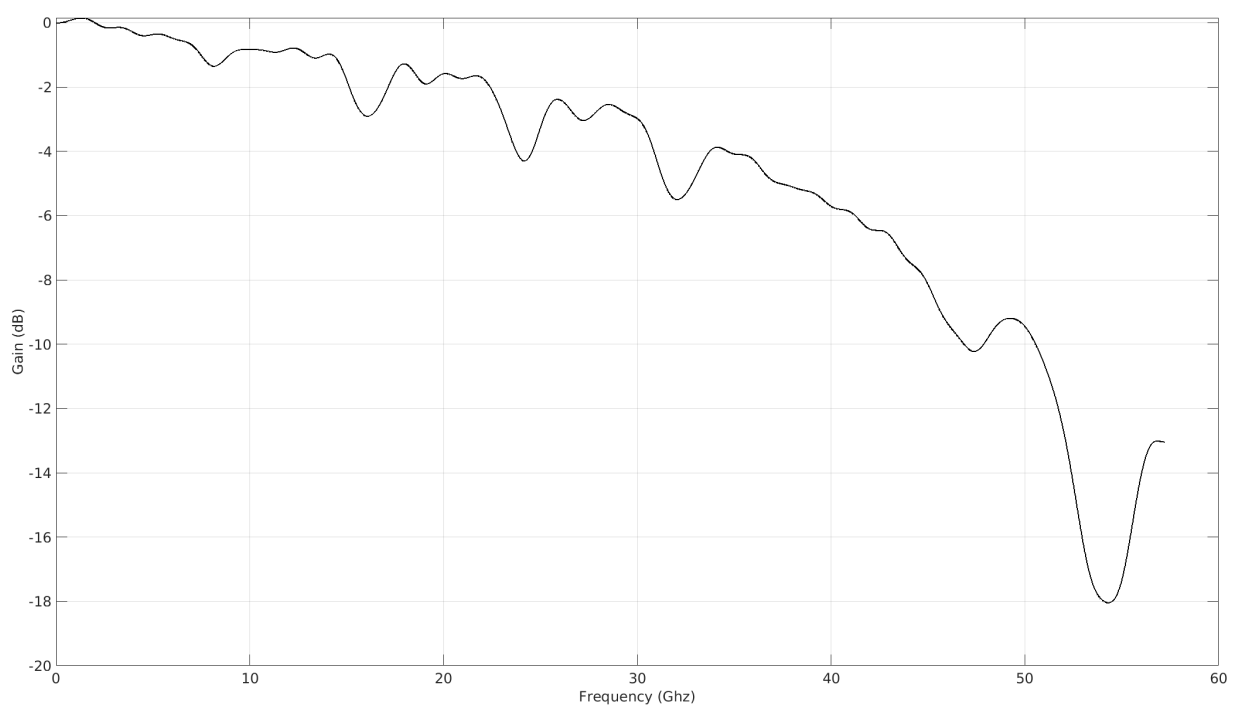

Figure 4.3: Electrical System Overall Magnitude Response

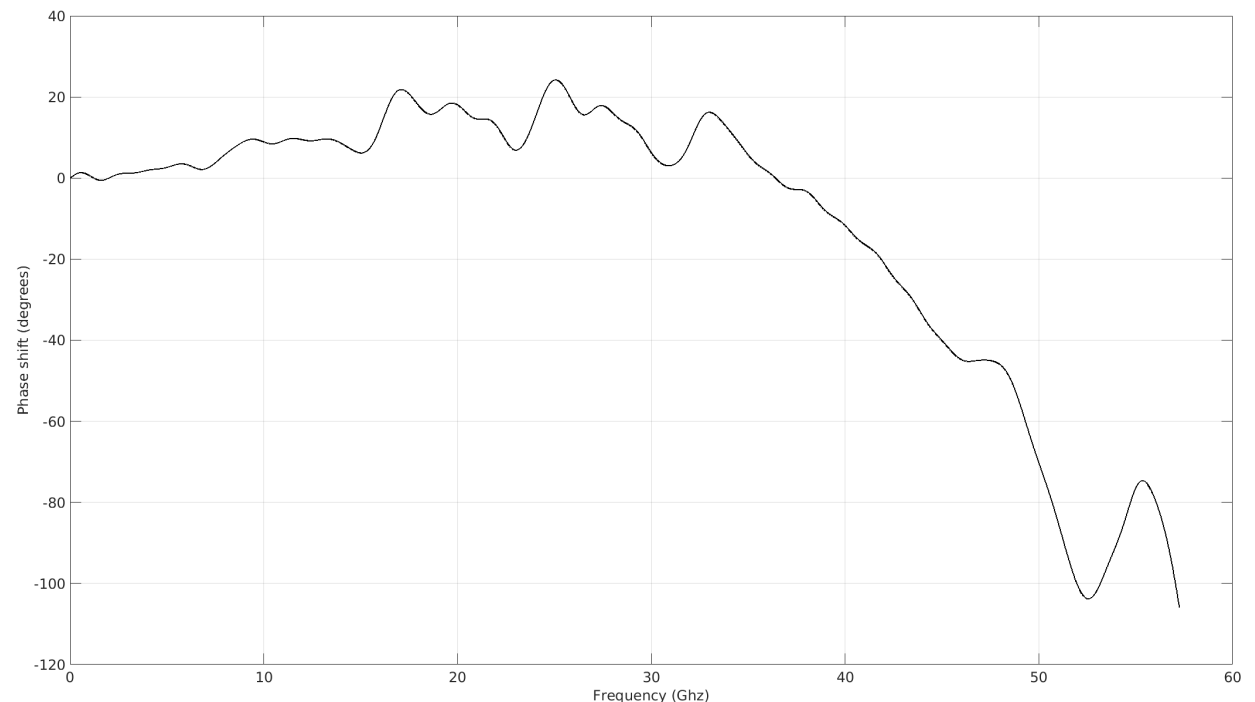

Figure 4.4: Electrical System Overall Phase Response 


$$
A_{\text {clip }}=\mu_{\text {desired }} \cdot \sigma^{2}
$$

Following clipping, the DMT time domain signal is scaled to fill the full dynamic range of the 8-bit DAC. The DAC is operating at $100+\mathrm{Gs} / \mathrm{s}$, where an oversampling ratio of 1.25 is used. Finally, the signal is uploaded to the transmit memory of the evaluation-board, where it will be applied at the DAC continuously and transmitted onto the channel to the receiver.

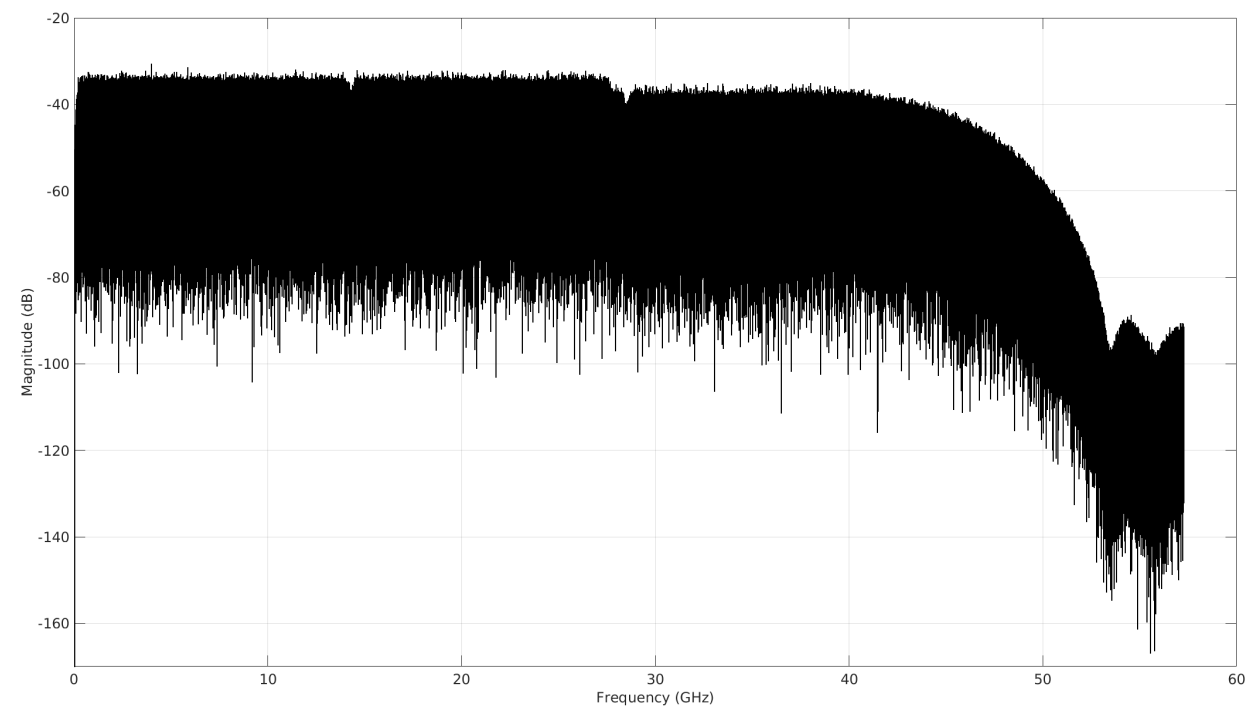

Figure 4.5: Electrical System Transmitted Spectrum

\subsubsection{Receiver}

At the receiver side, the received DMT time domain symbol is extracted from the receiver memory after having being continuously sampled by the 8-bit ADC at 100+ Gs/s. Due to this intermittent continuous transmission method, synchronization needs to be performed to align the transmitted waveform to that of the received as described in section 3.6.

After the received waveform is scaled to the maximum dynamic range of the ADC and is aligned to the transmitted waveform, it is passed through the FFT block to obtain the received constellation. Post-compensation, complex correction applied 
to the recieved constellation, is performed to compensate for deterministic rotation and/or scaling of the constellation. Finally, the constellation can be de-mapped back into bits where the BER can be analyzed.

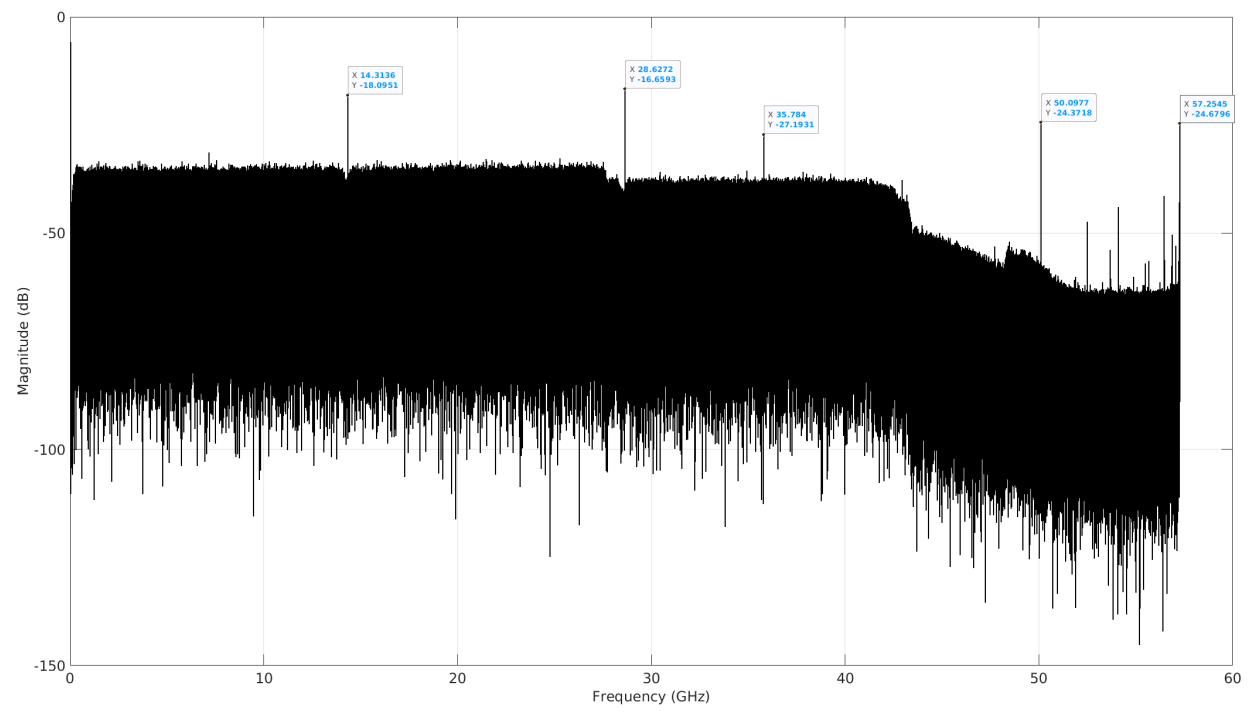

Figure 4.6: Electrical System Received Spectrum

\subsubsection{Results}

The main analysis metric performed on the overall system is the examining of the BER. Practically every optimization method discussed in chapter 3 is targeted at the main goal of reducing the errors in transmission by addressing various impairment mechanisms and maximising the throughput. Providing error free transmission is a requirement in any transmission system, resulting in the need for providing FEC. Obtaining a low error rate prior to using FEC allows the use of a lower strength FEC, minimizing the overhead it carries as well as the power consumption of the receiver.

Figure 4.5 shows the spectrum of the transmitted DMT signal. The oversampling ratio is evident due to the roll off at three quarters of the bandwidth (corresponding to an oversampling ratio of 1.25), as well as the ripples at high frequency due to the anti-aliasing filter.

The received spectrum can be seen in Figure 4.6 where a few strong spurs can be seen. Some of these correspond to the sampling frequency, such as the tones at $\frac{f s}{8}$ 
and $\frac{f_{s}}{4}$. The discussion of their causes are beyond this thesis due to the design of the chip being intellectual property belonging to the manufacturer. However, one can see the advantage of DMT in this cause by considering that the sub channel where these tones fall under will use a lower modulation scheme (seen in Chapter 3) to overcome this. In other systems, bandstop filters are likely required to overcome them, which carry their difficulties and disadvantages.

The benefits of bit-loading are apparent when looking at the BER plot, shown in Figure 4.7, whereby the BER is kept below the target error rate of $3.8 \times 10^{-3}$.

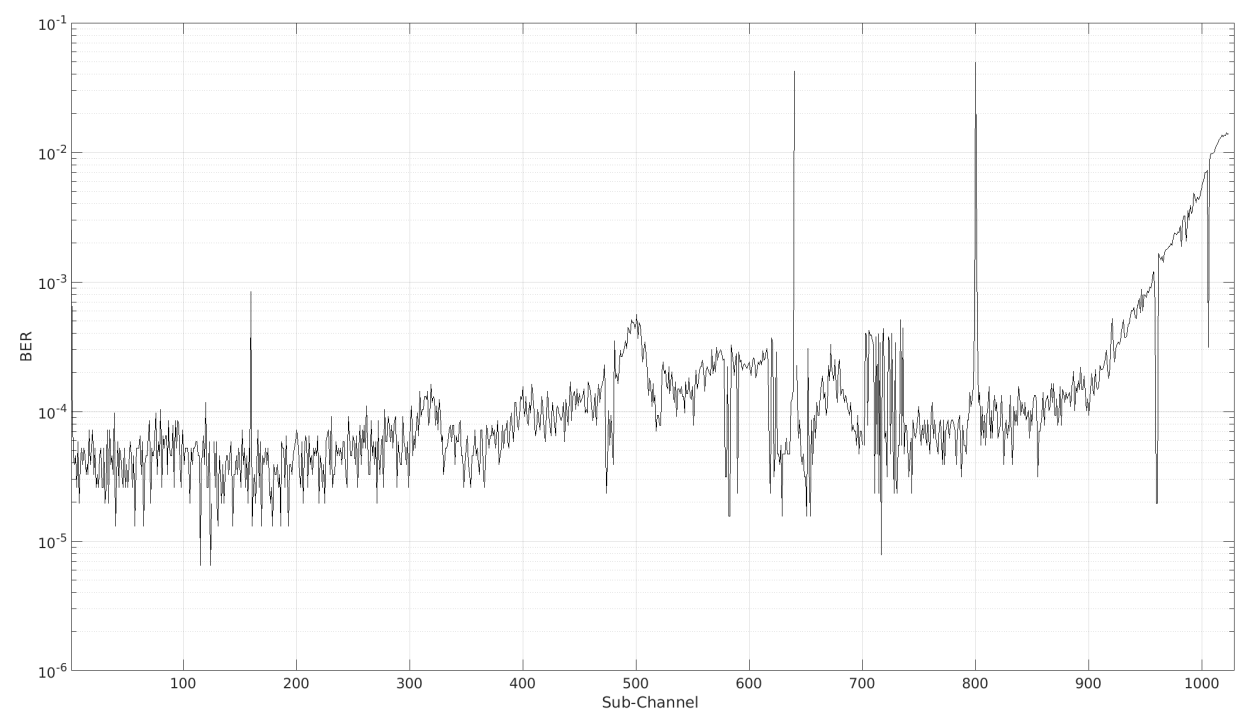

Figure 4.7: Electrical System BER Plot

A few selected received constellations are shown in Figures 4.8 to 4.21 where the modulation order ranges from 64-QAM down to 4-QAM. For sub channels falling at higher frequencies, the outermost symbols experience the largest error vectors, which can be seen by comparing their dispersion against symbols that fall towards the center of the constellation. One possible cause for this is the limited linearity of the system - symbols at higher amplitudes in frequency domain (prior to IFFT operation) result in higher amplitudes in the time domain DMT symbols, which undergoes non-linear behaviour through a limited linearity analog system [31] . This investigation is left for future work. A common solution for this that's used in commercial coherent optical transceivers, known as probabilistic shaping, is to minimize the visitation to 
the symbols on the outer limits of the constellation to minimize the errors caused by these points [32] [31], which can provide up to $15 \%$ increase in capacity. This can prove to be effective as the majority of the errors are caused by those symbols. The topic of probabilistic constellation shaping is also beyond the intentions of this work.

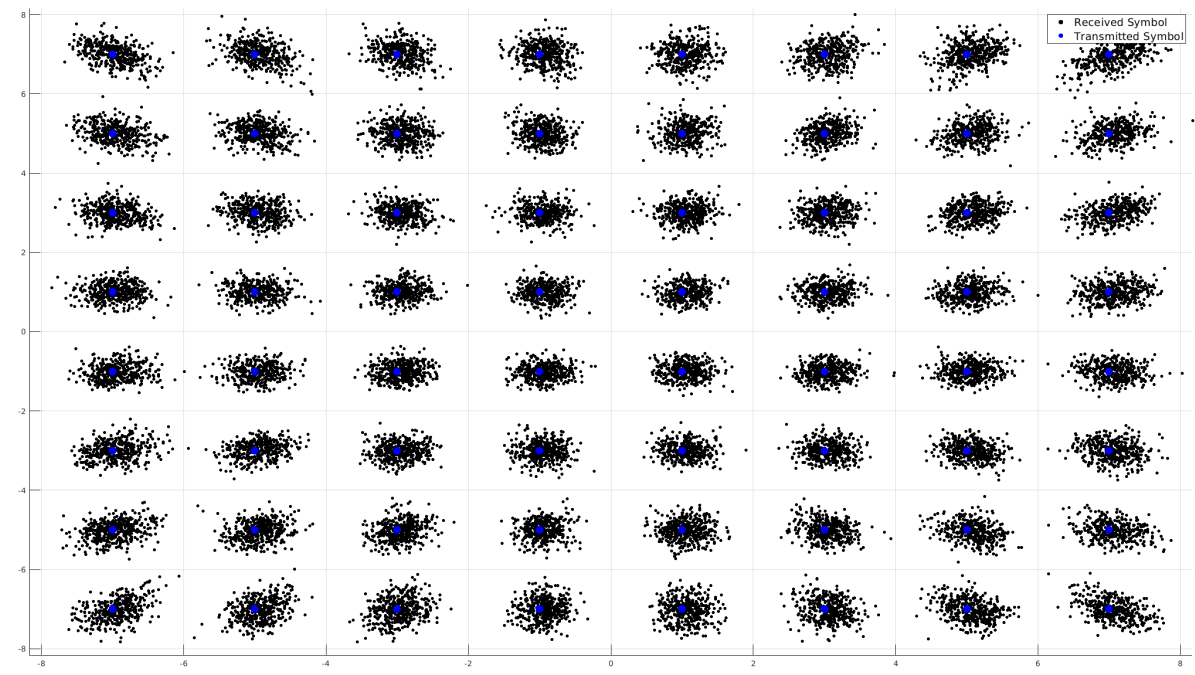

Figure 4.8: Rx Constellation Electrical System Ch 1004.4 GHz)

These constellations show that the entire spectrum can be used effectively for data transmission through the use of bit-loading and the robustness of DMT against variance in frequency response with the inclusion of strong spurs.

\subsection{Optical System}

\subsubsection{System Description}

For the case of an optical channel, an existing optical commercial transceiver was used to perform measurements. The majority of the measurements are performed similarly to the case of the electrical channel from the perspective of the pre and post processing in MATLAB. A key difference is the presence of electro-optic components 


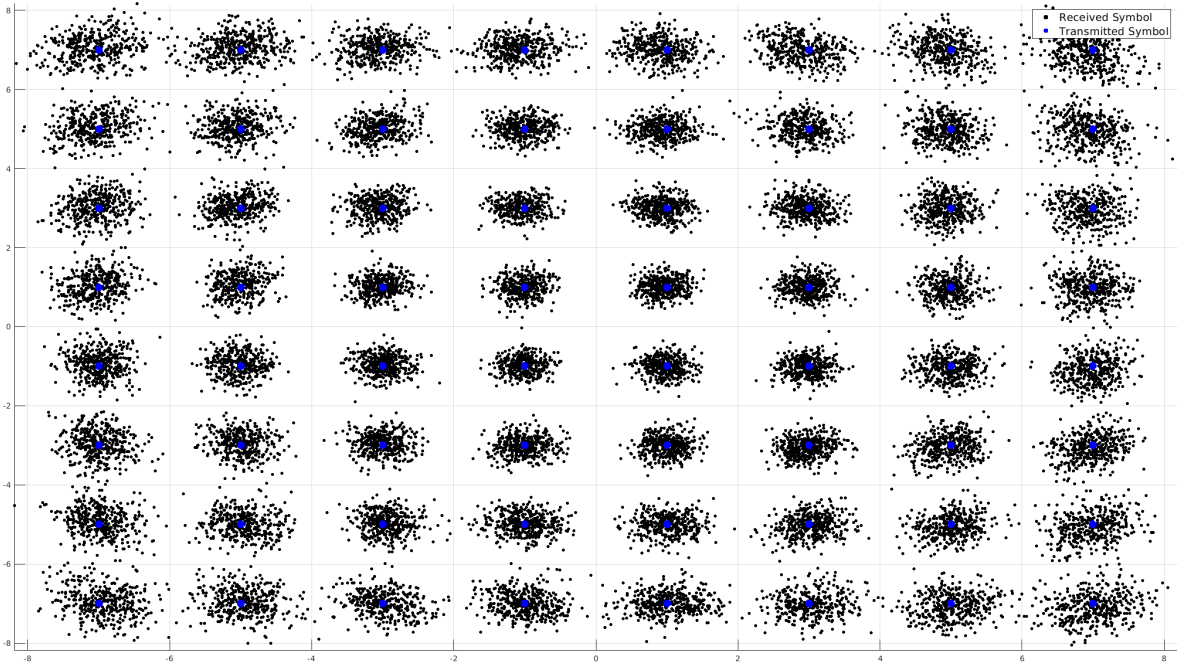

Figure 4.9: Rx Constellation Electrical System Ch 736 (32.9 GHz)

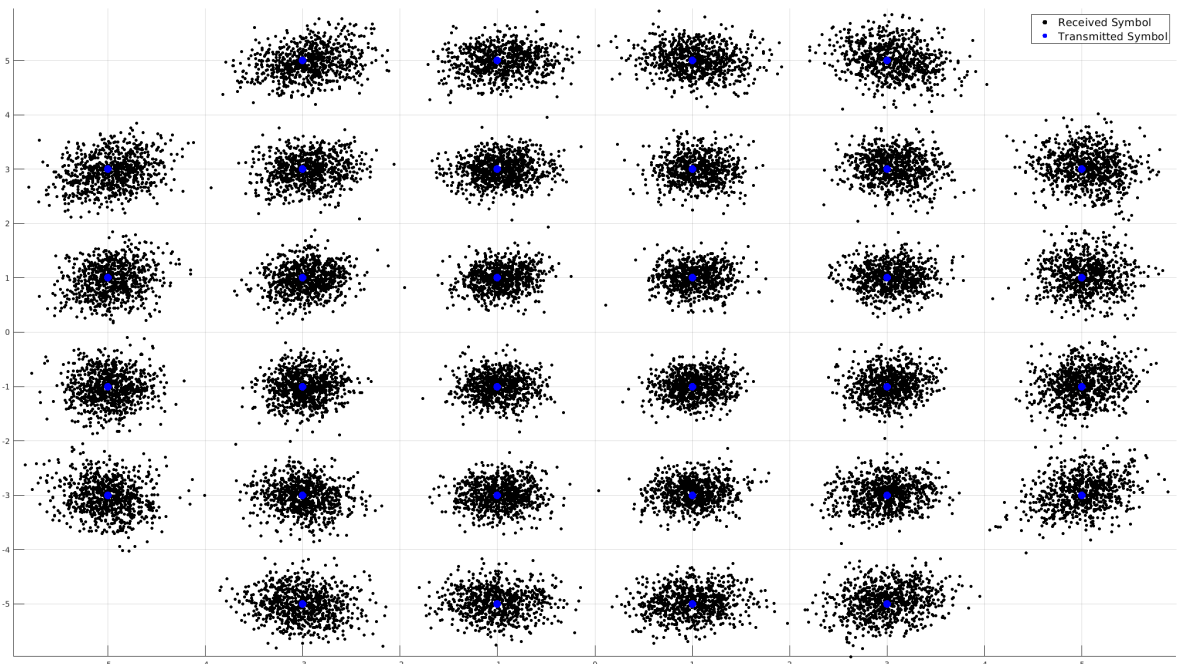

Figure 4.10: Rx Constellation Electrical System Ch 850 (38.0 GHz) 


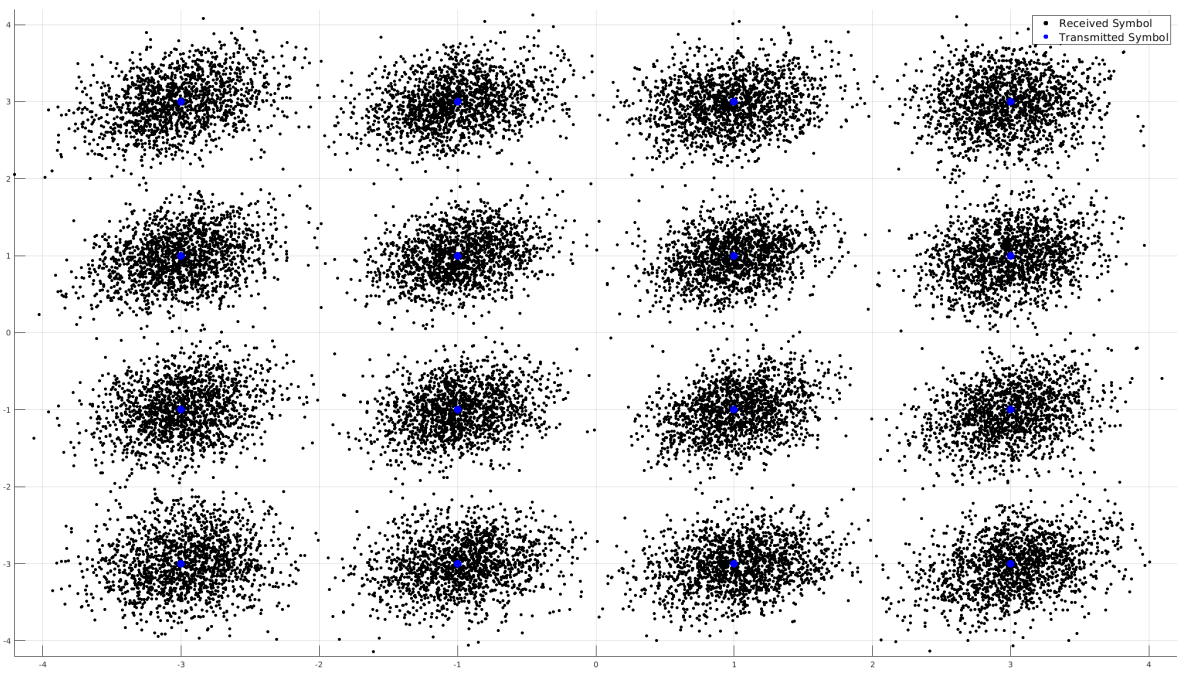

Figure 4.11: Rx Constellation Electrical System Ch 959 (42.9 GHz)

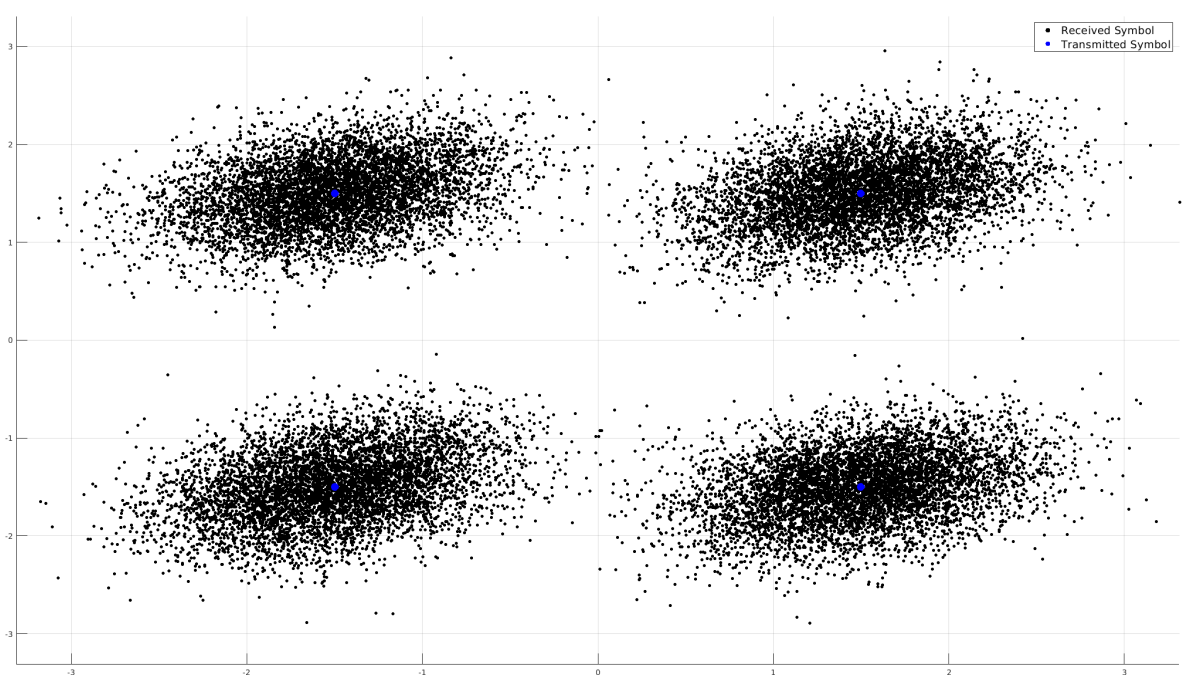

Figure 4.12: Rx Constellation Electrical System Ch 1006 (45.0 GHz) 
in order to transmit the signal through an optical channel. Another vital difference is that the system is considered a coherent system as there is a carrier recovery process that takes place in order to demodulate the data off the optical carrier, as opposed to a direct-detection method. As this system is a fully enclosed product that's commercially available, it also contains various bias loops and control circuitry that are not accessible to the user for tuning and cannot be discussed for the purpose of this work due to confidentiality. This placed a challenge for debugging and optimization as the circuitry is not designed to maintain conditions that are optimal for a signal with characteristics of a DMT signal.

The optical transceiver, or modem, uses a Micro Integrable Tunable Laser Assembly operating at in the C-band (1530nm - 156nm wavelength), where the transmitter and receiver are connected in loop-back using a single-mode fibre cable that is a few centimetres in length. It houses data converters with a sampling rate of $68.16 \mathrm{Gs} / \mathrm{s}$. As can be seen in Figure 4.13, the test setup for the optical channel contains many more components, each with their own intricacies and characteristics that are not configurable and not necessarily optimized for DMT transmission. However, despite this limitation, this work achieved a throughput that is $15 \%$ higher and a bit rate that is an order of magnitude lower than what the transceiver operates at. It should be noted that one tributary (single polarization and single quadrature) was used to perform the measurements. Details of the transmitter are not included in Figure 4.13 for the purposes of protecting intellectual property. The frequency response from the transmitter to the receiver can be seen in Figures 4.14 and 4.15 which include the data converters, channel, and the electro-optic components such as the modulator and the driver. Similarly to that of the electrical system, no additional impairments injected onto the signal such as noise as there are already sufficient impairments present which include the ones mentioned earlier in addition to the ones that are present as a result of the utilization of an optical carrier.

\subsubsection{Results}

Figure 4.16 shows the overall BER for the optical system measurement. A few selected received constellations are shown in Figures 4.17 to 4.21 where the modulation order ranges from 32-QAM down to 4-QAM. The inability to reach a modulation order of 


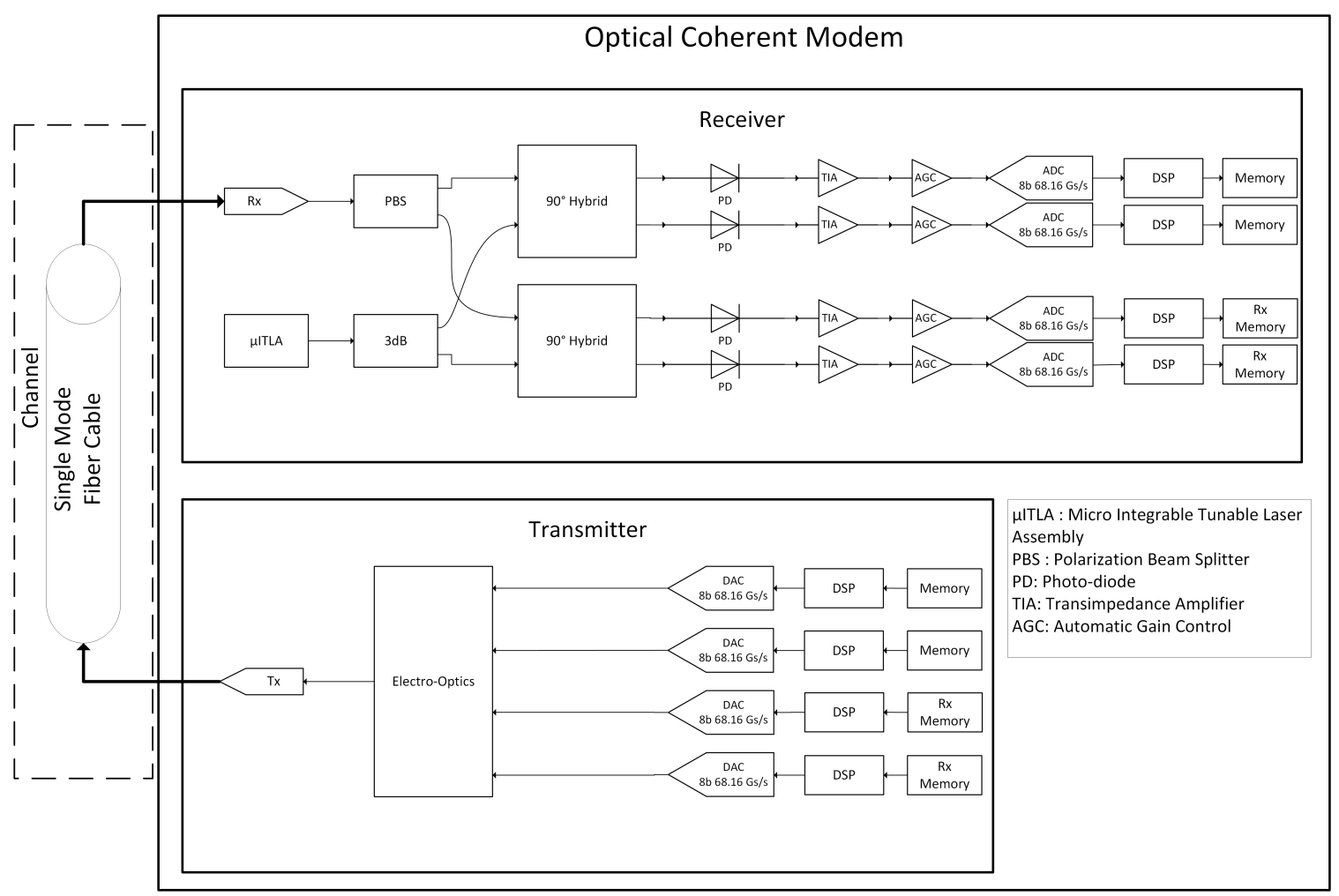

Figure 4.13: Optical System Test Setup Block Diagram

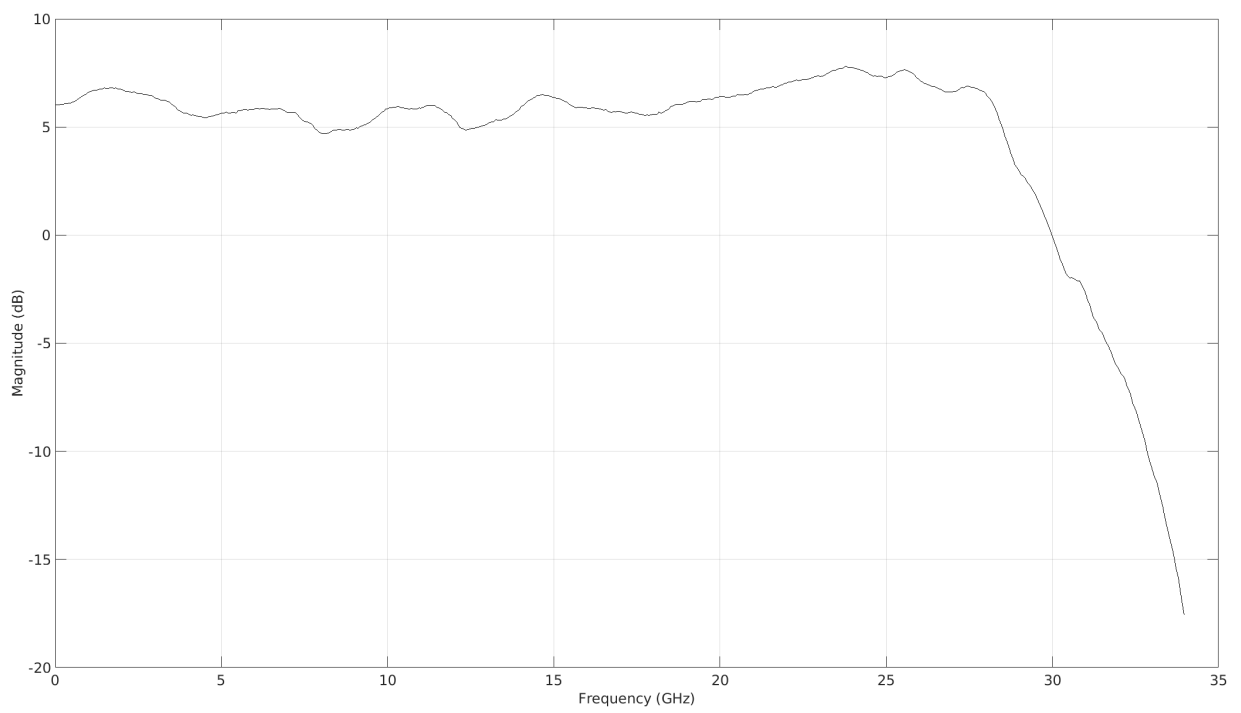

Figure 4.14: Optical System Overall Magnitude Response 


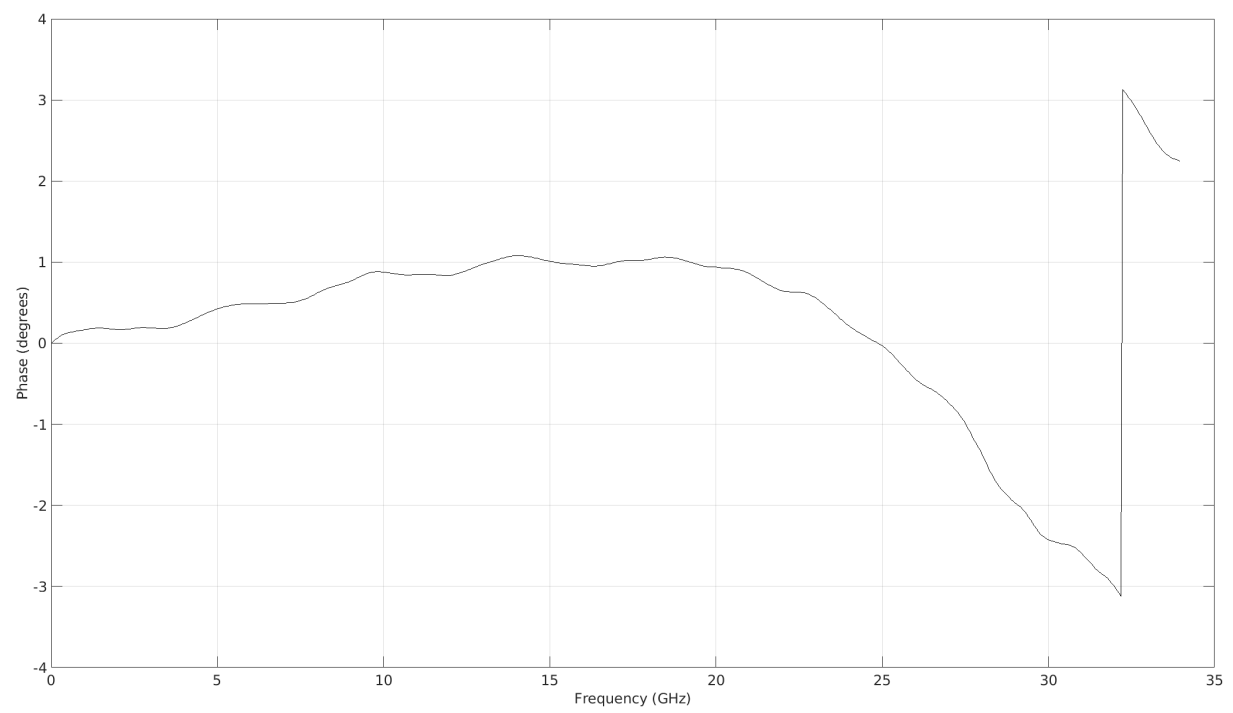

Figure 4.15: Optical System Overall Phase Response

64-QAM, as was done in the electrical system, is likely due to either the lower effective number of bits (ENOB) for the converters in the optical system, or higher thermal noise resulting in a lower SNR.

\subsection{Power Consumption Estimate}

In an attempt to accurately compare the performance of a DMT system against typically used PAM based coherent transceivers, a power consumption estimate will be calculated based on recently published state of the art work.

The major components in the DMT system designed in this work, found in Figures 3.1 and 3.2, are the IFFT/FFT, DAC, ADC, fractional oversampler, fractional decimator, scaling/clipping, and post-compensation. Note that pre-compensation was not used in this design. A re-configurable FFT design implemented in 40nm technology was used to estimate the FFT consumption in this design [33]. Scaling for frequency using Equation 3.1, was needed due to different FFT lengths requiring 


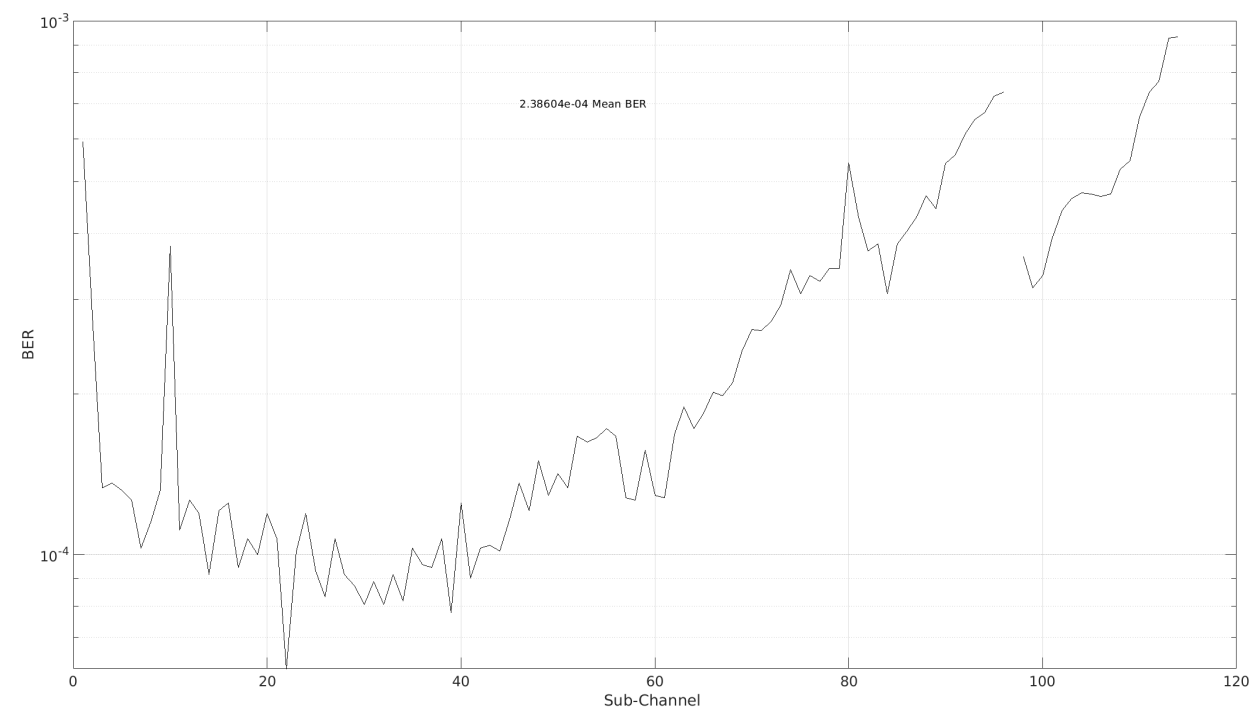

Figure 4.16: Optical System BER Plot

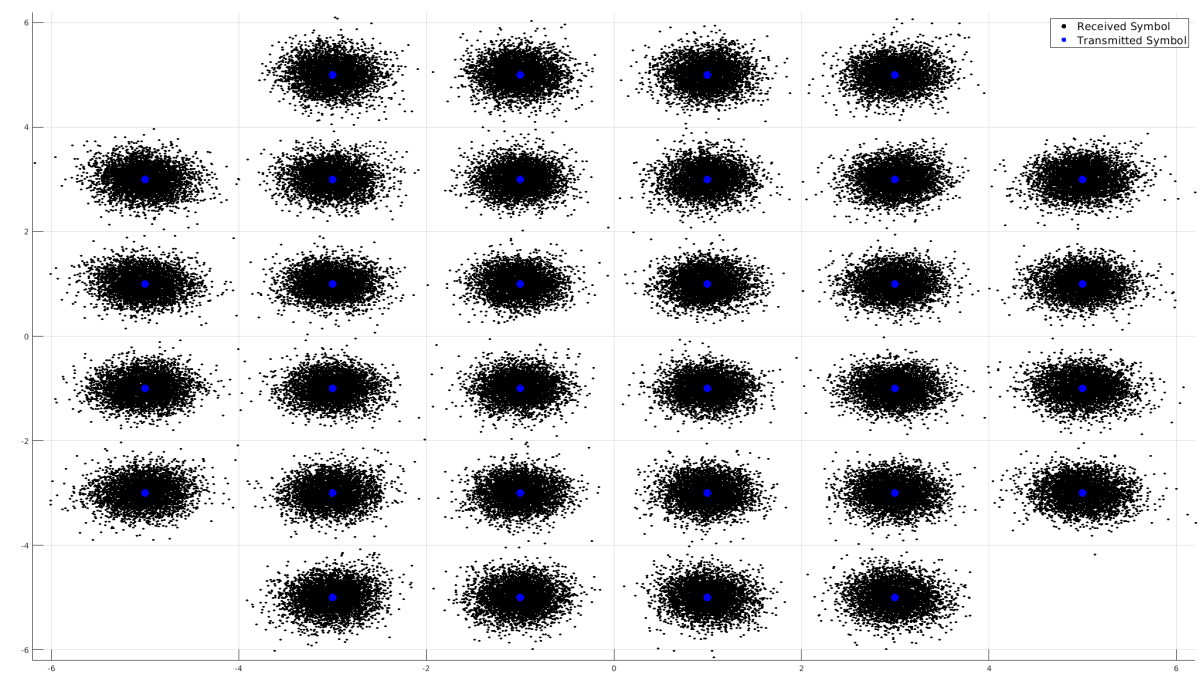

Figure 4.17: Rx Constellation Optical System Ch 25 (25.5 GHz) 


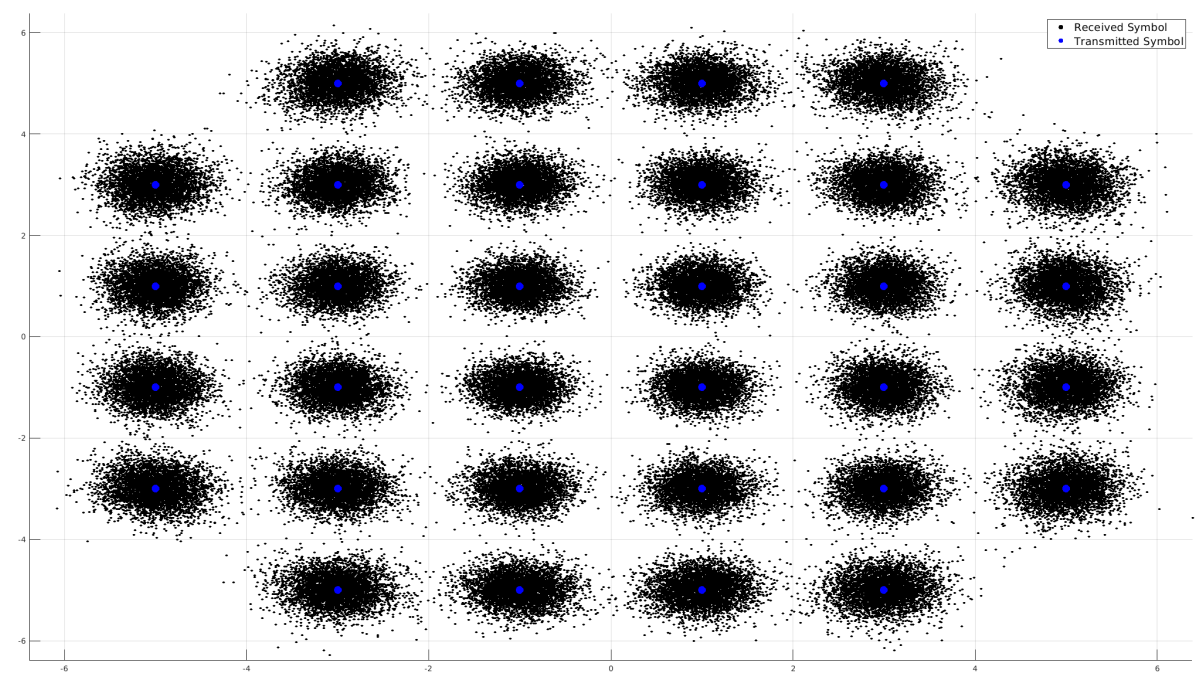

Figure 4.18: Rx Constellation Optical System Ch 75 (16.0 GHz)

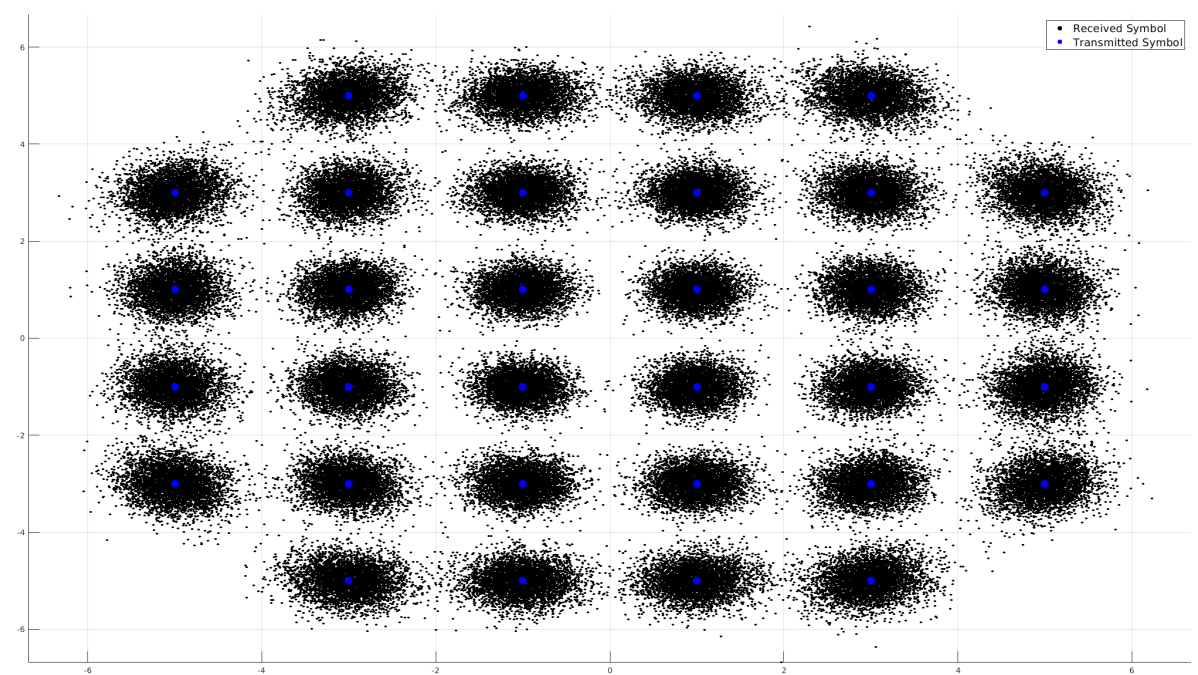

Figure 4.19: Rx Constellation Optical System Ch 95 (20.2 GHz) 


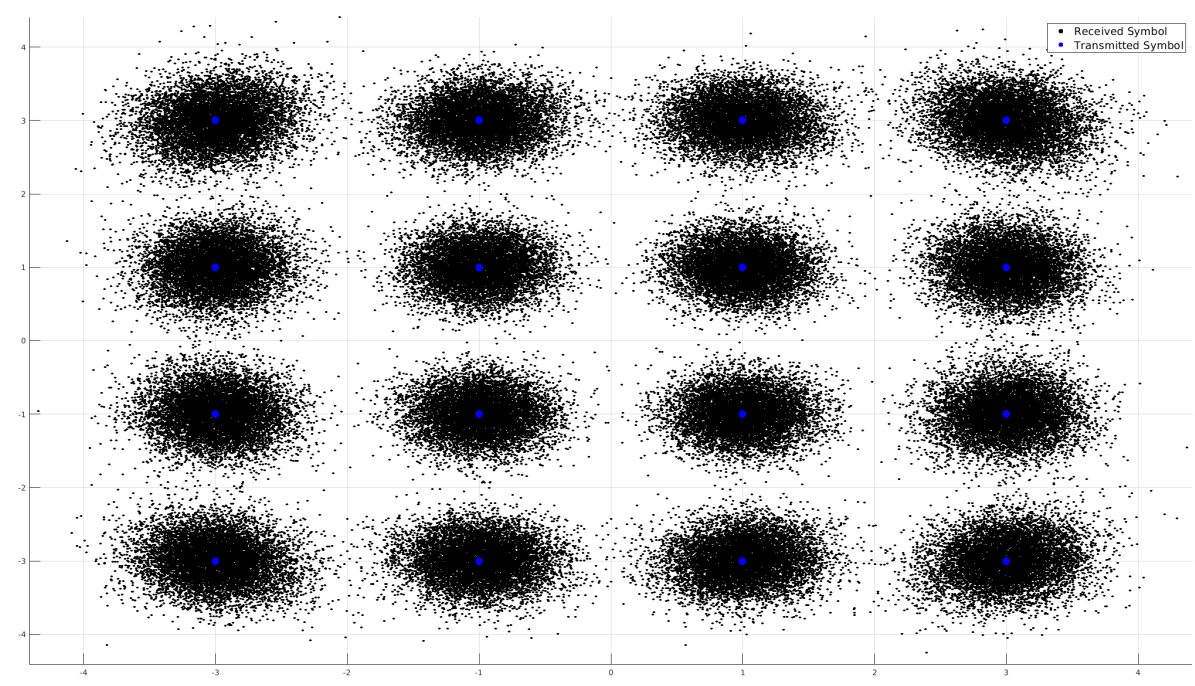

Figure 4.20: Rx Constellation Optical System Ch 105 (22.6 GHz)

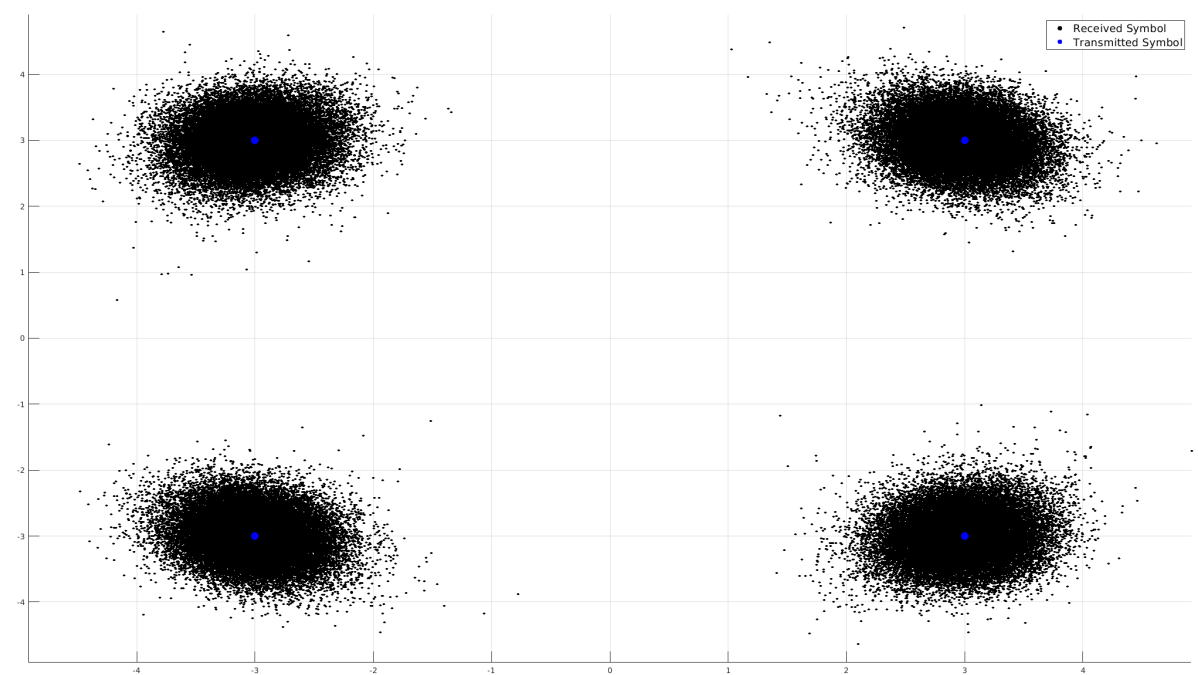

Figure 4.21: Rx Constellation Optical System Ch 125 (25.0 GHz) 
different operation frequency to deliver the same throughput. For the ADC, the Boris VLSI ADC survey [34] was used, where a linear extrapolation for the desired sampling frequency was performed to obtain an approximate power consumption of 1.25 watts. The DAC was not taken into account in this estimate as it is not part of a power consumption figures for transceivers in published work.

Table 4.1: Implemented Electrical DMT System Power Consumption Estimate

\begin{tabular}{|l|l|}
\hline \multicolumn{2}{|c|}{ Transmitter } \\
\hline Sub-Block & Power (Watts) \\
\hline IFFT & 0.0086 \\
\hline Scaling/Clipping & $*$ \\
\hline Fractional Oversampling & $* *$ \\
\hline Total Power (Watts) & 0.0086 \\
\hline Total Power (pJ/b) & 0.037 \\
\hline \multicolumn{2}{|c|}{ Receiver } \\
\hline Sub-Block & Power (Watts) \\
\hline ADC & 1.25 \\
\hline IFFT & 0.0086 \\
\hline Post-Compensation & $*$ \\
\hline Fractional Decimation & $*$ \\
\hline Total Power (Watts) & 1.26 \\
\hline Total Power (pJ/b) & 5.1 \\
\hline & \\
\hline
\end{tabular}

* Simple digital circuitry operating at a low frequency

** May require analog circuitry or new design techniques to achieve reasonably low power consumption

This results in a total transceiver power consumption estimate of $5.1 \mathrm{pJ} / \mathrm{b}$ compared to $14.1 \mathrm{pJ} / \mathrm{b}$ seen in Table 2.1 for a conventional transceiver implementation in a 
similar channel response design. The total for this work is also likely a over-estimate, since implementing the needed circuitry for a DMT system without unnecessary components and optimizing for DMT transmission will result in a lower power consumption. As a result the advantage in using DMT based systems is under-estimated here.

\subsection{Summary}

Table 4.2: Summary of Measurement Performance Metrics

\begin{tabular}{|l|l|l|}
\hline System & Electrical & Optical \\
\hline Data Rate $(\mathrm{Gbps})$ & 250 & 115.5 \\
\hline Average BER & $3.5 \times 10^{-4}$ & $2.39 \times 10^{-4}$ \\
\hline
\end{tabular}

This chapter presented the hardware setup used to measure the implemented DMT system as per the design techniques outlined in Chapter 3 and differentiated what was performed in DSP (MATLAB) and what was performed with existing hardware. This included the use of an existing evaluation test board and an existing optical transceiver for the electrical channel and optical channel cases respectively. Then, measured performance metrics and plots were given and summarized, including throughput, BER plots, and constellations. It should be noted that the performance achieved here does not represent the limitations of DMT, but rather the capabilities of using this modulation scheme and optimization techniques shown in 3 given the available hardware at hand for both the electrical and optical systems. The data rates achieved are 250 Gbps and 115.5 Gbps at a BER of $3.5 \times 10^{-4}$ and $2.39 \times 10^{-4}$ for the electrical and optical systems respectively. Finally, a power consumption estimate was performed , where a total power consumption of $5.1 \mathrm{pJ} / \mathrm{b}$ is estimated for this DMT transceiver in the electrical-channel case. 


\section{Chapter 5}

\section{Conclusion}

In this work, a DMT system is implemented using a combination of existing hardware and MATLAB Digital Signal processing. This work first introduced the current existing modulation schemes and hardware architectures in conventional high speed transceivers used in applications ranging from inter-chip high speed busses to metroscale data-center interconnects to shed light on how they compare to the implemented DMT system from the perspectives of performance and complexity. A thorough analysis of key aspects of a DMT system are analytically explored to reveal how they impact performance. This enabled the novelties in this work, which are the sequential combination of previously used optimization methods of sub-channel width selection, amplitude clipping, bit-loading, efficient simultaneous FFT, and post-compensation. The novelty of fractional oversampling, in addition to the preior optimization techniques, resulted in a system that rivals the performance of both recently published art in DMT and conventional transceivers. Through this analysis, a recipe for designing a DMT system can be adopted to suit the needs of transceivers that have varying requirements ranging from power consumption, channel loss, chip area, noise, and complexity.

Measurements were performed on both an electrical channel as well as an optical channel. The system implemented for the electrical channel is considered to be a direct-detection method (non-coherent), ie. a clock (carrier) recovery circuit is not required. However the optical channel system did comprise of a carrier re-

covery, classifying it as a coherent transceiver. This was done on the basis of the available hardware. Finally, a power consumption estimate is derived using state of the art published work that incorporates a few of the major components needed for DMT to shed light on all the advantages and disadvantages of this work compared 
to conventional transceivers.

The maximum spectral efficiency achieved was $5.82 \mathrm{~b} / \mathrm{s} / \mathrm{Hz}$, with a data rate of 250 Gbps at a $3.5 \times 10^{-4} \mathrm{BER}$ and an estimated power consumption of $5.1 \mathrm{pJ} / \mathrm{b}$. This proves to demonstrate the advantages of DMT over conventional amplitude modulation based transceivers that are higher in complexity, consume more power per bit, and lack the robustness available in DMT in which it can be deployed for various applications and channels of varying responses without major changes to the design.

A few key parameters of the system were investigated in order to arrive at an optimal solution in terms of throughput and error rate. The effects of clipping and scaling were measured in order confirm the theoretically expected behaviour. The selection of the number of sub-channel was also thoroughly examined. In addition to that, this work introduced previously unseen technique of fractional oversampling in DMT to enable a finer trade-off between BER and throughput as well as reduce quantization noise. This is tremendously advantageous depending on the characteristics of the frequency response of the system as there is a greater control in which portions of the spectrum to use while benefiting from the benefit of oversampling (increased SNR). At the time of writing, a publication has been submitted to the ISSCC 20201 and a patent application is in process for fractional oversampling in DMT. To address efficiency of hardware implementation, a technique to efficiently use the Fourier Transform hardware is also demonstrated. These techniques allowed the resultant optimal performance given the hardware at hand that is not optimized for DMT.

Tables 5.1 and 5.2 summarize the final results obtained in this work and compares it to that of other published work on DMT. For the case of the electrical-channel implementation, this work demonstrates almost a 5x throughput and a $15 \%$ spectral efficiency, where the spectral efficiency achieved is the highest seen in literature. It's previously shown in this work that the power estimate in this work is an over-estimate, thus building this transceiver is required in order to compare power consumption. Doing so according to the work implemented in this thesis would demonstrate higher performance and a lower power consumption due to the dedicated specialized hardware. As for the case of the optical-channel implementation, it can be seen that despite the reduced capability in configuring the system due to the hardware being an enclosed transceiver, the measurements proved to be comparable to other recent 
published work.

Table 5.1: Performance summary and comparison of this work to published electrical-channel DMT

\begin{tabular}{|c|c|c|}
\hline & {$[35]$} & This Work \\
\hline Data Rate (Gbps) & 56 & 250 \\
\hline Pre-FEC BER & $<1 \times 10^{-6}$ & $3.5 \times 10^{-4}$ \\
\hline Bandwidth $(\mathrm{GHz})$ & 11.2 & 42.94 \\
\hline Converter Sampling Rate Gs/s & 22.4 & $100+$ \\
\hline FFT Length & 32 & 1024 \\
\hline Sub-Channel Bandwidth (MHz) & 350 & 42 \\
\hline Channel Loss (dB) & 18 & 18 \\
\hline Spectral Efficiency (b/s/Hz) & 5.0 & 5.82 \\
\hline Power Consumption (pJ/b) & 2.9 & 5.1 \\
\hline
\end{tabular}

\subsection{Trade-Offs Discussion}

Oversampling has the drawback of reducing the total throughput by the oversampling ratio $n_{O S R}$, however it can be very advantageous depending on the system overall frequency response and the BER seen. The shape of the frequency response, more specifically the slope, will dictate what portions of the spectrum are best suited and the optimal OSR to use. Coupled with the ability to use a fractional OSR, one can more finely balance trading off throughput for BER. In some scenarios, oversampling can even provide the ability to use a higher order modulation scheme that negates some or all the throughput reduction caused by the use of oversampling, whilst still maintaining a BER below the desired. Figure 3.11 showed that the largest leap occurred when going from an OSR of 1 to an OSR of 1.25, whereby the portion of the spectrum with the largest slope with respect to channel loss, seen in Figure 4.3, is observed and avoided through the use of oversampling. 
Table 5.2: Performance summary and comparison of this work to published opticalchannel DMT

\begin{tabular}{|c|c|c|c|c|c|}
\hline & {$[36]$} & {$[37]$} & {$[38]$} & {$[39]$} & This work \\
\hline Data Rate (Gbps) & 300 & 400 & 215 & 250 & 115 \\
\hline Pre-FEC BER & $2.6 \times 10^{-2}$ & NA & $2.4 \times 10^{-2}$ & $4.5 \times 10^{-3}$ & $3.5 \times 10^{-4}$ \\
\hline$\lambda$-Band & O-Band & C-Band & C-Band & C-Band & C-Band \\
\hline Bandwidth (GHz) & 63 & 80 & 46.24 & 46 & 25.56 \\
\hline Converter Sampling Rate Gs/s & 160 & NA & 92 & 92 & 68.16 \\
\hline FFT Length & 256 & 256 & 1024 & 1024 & 1024 \\
\hline Sub-Channel Bandwidth (MHz) & 246 & 313 & 90 & 45 & 104 \\
\hline Channel Loss (dB) & NA & NA & NA & $\sim 22$ & 17 \\
\hline Spectral Efficiency (b/s/Hz) & 4.8 & 5 & 4.7 & 4.9 & 4.51 \\
\hline
\end{tabular}

A second trade-off to mention is the increase in chip area and possible powerconsumption increase as the sub-channel number increases. This would require specific FFT hardware to be designed and synthesized for different FFT length operating at different frequencies and compared in order to derive a definitive answer and a possible optimal point. The advantage of using a higher sub-channel count is the increased granularity for bit-loading, which can lead to a higher throughput and lower $\mathrm{BER}$, in addition to an improved post-compensation vector.

Another critical trade-off to be taken into account is the increased PAPR as the distance between QAM constellation points increases. This has the advantage of increasing resilience to thermal noise, but may degrade overall BER as a result of increased PAPR. This phenomena was observed, however the analysis and optimization of this is considered to be beyond this work and assigned to future work.

\subsection{Future Work}

The work done in this thesis may be extended by taking this design into a fully formed enclosed product. This will require the implementation and optimization of a few key sub-components that were replaced with MATLAB offline processing in this work. These include the FFT, fractional oversampling/decimation, post-compensation, and 
scaling/clipping. In addition to that, various circuitry that exists in the current hardware implementation can be removed or re-designed to target DMT transmission. A result of that would be improved performance, reduced area and power consumption of the integrated circuit as well as a more detailed comparison to conventional transceivers.

As discussed earlier, an analysis of the trade-off between the BER and constellation symbol spacing needs to be conducted and experimented with for different frequency response and thermal noise conditions to provide a deeper understanding on how to find an optimal point for a given application.

With the advancements of technology scaling, the reduction in power consumption or area will provide a tremendous advantage to DMT, as a good portion of the circuitry falls under the digital domain that typically benefits from technology node scaling. This may provide the power budget to research the usage of high speed digital time domain equalizers. Due to technology scaling, using FFTs of larger sizes than the ones used in this work should also be further investigated.

One area that may be of importance is the effect of the CP on the PAPR, and how that affects BER.

Finally, an area that has not been explored in literature is the use of a CTLE in the analog domain (prior to the ADC from Figure 2.7) to compensate for the frequency response, more specifically the attenuation of mid and high frequency components. This would effectively split the task of post-compensation between that done in the digital domain (frequency domain) as seen in this work and the analog domain (time domain). This may provide improvements in the error rate, thereby strengthening the case for using DMT based transceivers over conventional PAM-based coherent transceiver. 


\section{List of References}

[1] "Global Interconnection Index : Measuring the Growth of the Digital Economy," tech. rep., Equinox. https://equinix.box.com/shared/static/ ftumr3bnqq455zfnb0c3lm5lmnffa8f1.pdf, Date Accessed: 2020-06-20.

[2] "Scaling the Compute and High Speed Networking Needs of the Data Center With Silicon Photonics," tech. rep., Intel. https://www . intel.fr/content/dam/www/public/us/en/documents/product-briefs/ market-focus-robert-blum.pdf, Date Accessed: 2020-07-21.

[3] H. Zhang, "PAM4 Signaling for 56G Serial Link Applications," DesignCon, 2016. https://www.xilinx.com/publications/events/designcon/ 2016/slides-pam4signalingfor56gserial-zhang-designcon.pdf, Date Accessed: 2020-09-09.

[4] "OIF Hot Topic Fact Sheet - Common Electrical I/O (CEI)-112." https: //www . oiforum. com/wp-content/uploads/OIF-Hot-Topic-Fact-Sheet_ CEI_FINAL.pdf, Date Accessed: 2020-09-09.

[5] T. T. Ha., Theory and Design of Digital Communication Systems. Cambridge University Press, 2011.

[6] X. Shih, H. Chou, and Y. Liu, "VLSI Design and Implementation of Reconfigurable 46-Mode Combined-Radix-Based FFT Hardware Architecture for 3GPPLTE Applications," IEEE Transactions on Circuits and Systems I: Regular Papers, vol. 65, pp. 118-129, Jan 2018.

[7] "Visual Networking Index Complete Forecast Highlights," tech. rep., Cisco. https://www.cisco.com/c/dam/m/en_us/solutions/service-provider/ vni-forecast-highlights/pdf/Global_2020_Forecast_Highlights.pdf, Date Accessed: 2020-09-20.

[8] "2019 Ethernet Roadmap." https://ethernetalliance.org/technology/ 2019-roadmap/, Date Accessed: 2020-04-17.

[9] Y. Krupnik et al., "112-Gb/s PAM4 ADC-Based SERDES Receiver With Resonant AFE for Long-Reach Channels," IEEE Journal of Solid-State Circuits, vol. 55, no. 4, pp. 1077-1085, 2020.

[10] B. Yoo et al., "6.4 A 56Gb/s 7.7mW/Gb/s PAM-4 Wireline Transceiver in 10nm FinFET Using MM-CDR-Based ADC Timing Skew Control and Low-Power DSP 
with Approximate Multiplier," in 2020 IEEE International Solid- State Circuits Conference - (ISSCC), pp. 122-124, 2020.

[11] E. Depaoli et al., "A 4.9pJ/b 16-to-64Gb/s PAM-4 VSR transceiver in 28nm FDSOI CMOS," in 2018 IEEE International Solid - State Circuits Conference (ISSCC), pp. 112-114, 2018.

[12] P. W. de Abreu Farias Neto et al., "A 112-134-Gb/s PAM4 Receiver Using a 36-Way Dual-Comparator TI-SAR ADC in 7-nm FinFET," IEEE Solid-State Circuits Letters, vol. 3, pp. 138-141, 2020.

[13] J. Hudner et al., "A 112GB/S PAM4 Wireline Receiver Using a 64-Way TimeInterleaved SAR ADC in 16NM FinFET," pp. 47-48, 2018.

[14] A. Ghiasi, "Large data centers interconnect bottlenecks," Opt. Express, vol. 23, pp. 2085-2090, Feb 2015.

[15] Z. Li et al., "Comparison of digital signal-signal beat interference compensation techniques in direct-detection subcarrier modulation systems," Opt. Express, vol. 24, pp. 29176-29189, Dec 2016.

[16] X. Pang et al., "200 Gbps/Lane IM/DD Technologies for Short Reach Optical Interconnects," Journal of Lightwave Technology, vol. 38, no. 2, pp. 492-503, 2020.

[17] A. Gatto, M. Rapisarda, P. Parolari, and P. Boffi, "Discrete multitone modulation for short-reach mode division multiplexing transmission," Journal of Lightwave Technology.

[18] M. Chen, J. He, and L. Chen, "Real-Time Demonstration of 1024-QAM OFDM Transmitter in Short-Reach IMDD Systems," IEEE Photonics Technology Letters, vol. 27, no. 8, pp. 824-827, 2015.

[19] K. Tan et al., "A 112-GB/S PAM4 Transmitter in 16NM FinFET," in 2018 IEEE Symposium on VLSI Circuits, pp. 45-46, 2018.

[20] S. Karabetsos, E. Pikasis, T. Nikas, A. Nassiopoulos, and D. Syvridis, "DFTSpread DMT Modulation for 1-Gb/s Transmission Rate Over $100 \mathrm{~m}$ of 1-mm SI-POF," IEEE Photonics Technology Letters, vol. 24, no. 10, pp. 836-838, 2012.

[21] D. J. G. Mestdagh, P. Spruyt, and B. Biran, "Analysis of clipping effect in DMT-based ADSL systems," in Proceedings of ICC/SUPERCOMM'94 - 1994 International Conference on Communications, pp. 293-300 vol.1, 1994.

[22] R. Prasad, OFDM for Wireless Communications Systems. Artech House, 2004.

[23] D. Mestdagh, "Calculation of ADC resolution for DMT modulation," 1995.

[24] J. Hou, X. Zhao, F. Gong, F. Hui, and J. Ge, "PAPR and PICR Reduction of OFDM Signals With Clipping Noise-Based Tone Injection Scheme," IEEE Transactions on Vehicular Technology, vol. 66, no. 1, pp. 222-232, 2017. 
[25] J. Rogers, C. Plett, and I. Marsland, Radio Frequency System Architecture and Design. Artech House, 2013.

[26] R. Prasad, Integrated Circuit Design for High-Speed Frequency Synthesis. Artech House, 2004.

[27] N. Takahashi, D. Takago, S. Matsumura, and T. Takebe, "Efficient multistage implementation of rational sampling rate converter," in 2013 European Conference on Circuit Theory and Design (ECCTD), pp. 1-4, 2013.

[28] K. S. Al-Mawali, A. Z. Sadik, and Z. M. Hussain, "Simple discrete bit-loading for OFDM systems in power line communications," in 2011 IEEE International Symposium on Power Line Communications and Its Applications, pp. 267-270, 2011.

[29] R. Matusiak, "Implementing Fast Fourier Transform Algorithms of Real-Valued Sequences with the TMS320 DSP Platform," 2001. http://www.ti.com/lit/ an/spra291/spra291 . pdf?\&ts=1589813850966, Date Accessed: 2020-05-10.

[30] M. Chagnon, T. A. Eriksson, F. Buchali, H. Bülow, and S. ten Brink, "Single Carrier $168 \mathrm{~Gb} / \mathrm{s}$ PAM8 over $80 \mathrm{~km}$ Below HD-FEC Using Simple Receiver Equalization for Data Centre Interconnects," in 2017 European Conference on Optical Communication (ECOC), pp. 1-3, 2017.

[31] F. Buchali et al., "Experimental demonstration of capacity increase and rateadaptation by probabilistically shaped 64-QAM," in 2015 European Conference on Optical Communication (ECOC), pp. 1-3, 2015.

[32] Q. Guo, W. Peng, Y. Cui, and Y. Bai, "Multi-dimensional probabilistic shaping for higher fibre nonlinearity tolerance," in 45th European Conference on Optical Communication (ECOC 2019), pp. 1-3, 2019.

[33] X. Shih, H. Chou, and Y. Liu, "VLSI Design and Implementation of Reconfigurable 46-Mode Combined-Radix-Based FFT Hardware Architecture for 3GPPLTE Applications," IEEE Transactions on Circuits and Systems I: Regular Papers, vol. 65, no. 1, pp. 118-129, 2018.

[34] B. Murmann, "ADC Performance Survey 1997-2020," 2020. http://web. stanford.edu/ murmann/publications/ADCsurvey_rev20200802.xls, Date Accessed: 2020-07-23.

[35] G. Kim et al., "A 161-mW 56-Gb/s ADC-Based Discrete Multitone Wireline Receiver Data-Path in 14-nm FinFET," IEEE Journal of Solid-State Circuits, vol. 55, no. 1, pp. 38-48, 2020.

[36] H. Yamazaki et al., "300-Gbps Discrete Multi-tone Transmission Using DigitalPreprocessed Analog-Multiplexed DAC with Halved Clock Frequency and Suppressed Image," in ECOC 2016 - Post Deadline Paper; 42nd European Conference on Optical Communication, pp. 1-3, 2016.

[37] H. Yamazaki et al., "IMDD Transmission at Net Data Rate of $333 \mathrm{~Gb} / \mathrm{s}$ Using Over-100-GHz-Bandwidth Analog Multiplexer and Mach-Zehnder Modulator," Journal of Lightwave Technology, vol. 37, no. 8, pp. 1772-1778, 2019. 
[38] L. Zhang et al., "Nonlinearity-aware 200 Gbits/s DMT transmission for C-band short-reach optical interconnects with a single packaged electro-absorption modulated laser," Opt. Lett., vol. 43, pp. 182-185, Jan 2018.

[39] L. Zhang, J. Wei, N. Stojanovic, C. Prodaniuc, and C. Xie, "Beyond 200-Gb/s DMT Transmission Over 2-km SMF Based on A Low-Cost Architecture with Single-Wavelength, Single-DAC/ADC and Single-PD," in 2018 European Conference on Optical Communication (ECOC), pp. 1-3, 2018. 


\section{Appendix A}

\section{Efficient Simultaneous FFT of Real Signals}

Here is the derivation.

Suppose $\mathrm{h}(\mathrm{t})$ is a complex function in time domain made up of a real component $h_{r}(t)$ and an imaginary component $h_{i}(t)$ :

$$
\begin{gathered}
h(t)=h_{r}(t)+j h_{i}(t) \\
H(n)=\sum_{0}^{N-1}\left[h_{r}(k)+j h_{i}(k)\right] e^{-j 2 \pi n k / N} d t \\
=\sum_{0}^{N-1}\left[h_{r}(k) \cos \left(\frac{2 \pi n k}{N}\right)+h_{i}(k) \sin \left(\frac{2 \pi n k}{N}\right)\right] d t \\
-j \sum_{0}^{N-1}\left[h_{r}(k) \sin \left(\frac{2 \pi n k}{N}\right)+h_{i}(k) \cos \left(\frac{2 \pi n k}{N}\right)\right] d t \\
=R(n)+j I(n)
\end{gathered}
$$

If $\mathrm{h}(\mathrm{k})$ is purely real then equation A.6 becomes :

$$
R_{e}(n)=\sum_{0}^{N-1} h_{r}(k) \cos \left(\frac{2 \pi n k}{N}\right) d t
$$




$$
I_{o}(n)=-j \sum_{0}^{N-1}\left[h_{r}(k) \sin \left(\frac{2 \pi n k}{N}\right) d t\right.
$$

If $\mathrm{h}(\mathrm{k})$ is purely imaginary then equation A.6 becomes :

$$
\begin{aligned}
& R_{o}(n)=\sum_{0}^{N-1} h_{i}(k) \cos \left(\frac{2 \pi n k}{N}\right) d t \\
& I_{e}(n)=\sum_{0}^{N-1} h_{i}(k) \cos \left(\frac{2 \pi n k}{N}\right) d t
\end{aligned}
$$

Waveform Decomposition

Suppose $\mathrm{y}(\mathrm{k})$ is constructed

$$
\begin{aligned}
Y(n) & =H(n)+j G(n) \\
& =\left[H_{r}(n)+j H_{i}(n)\right]+j\left[G_{r}(n)+j G_{i}(n)\right] \\
& =\left[H_{r}(n)-G_{i}(n)\right]+j\left[H_{i}(n)+G_{r}(n)\right] \\
& =R(n)+j I(n)
\end{aligned}
$$

Decomposing gives :

$$
\begin{aligned}
Y(n) & =\left(\frac{R(n)}{2}+\frac{R(N-n)}{2}\right)+\left(\frac{R(n)}{2}-\frac{R(N-n)}{2}\right) \\
& +j\left(\frac{I(n)}{2}+\frac{I(N-n)}{2}\right)+j\left(\frac{I(n)}{2}-\frac{I(N-n)}{2}\right)
\end{aligned}
$$

From equations $6 / 45$ and 6.46 :

$$
\begin{aligned}
H(n) & =R_{o}(n)+j I_{e}(n) \\
& =\left(\frac{R(n)}{2}+\frac{R(N-n)}{2}\right)+j\left(\frac{I(n)}{2}-\frac{I(N-n)}{2}\right)
\end{aligned}
$$




$$
\begin{aligned}
j G(n) & =R_{o}(n)+j I_{e}(n) \\
G(n) & =I_{e}(n)-j R_{o}(n) \\
& =\left(\frac{I(n)}{2}+\frac{I(N-n)}{2}\right)-j\left(\frac{R(n)}{2}-\frac{R(N-n)}{2}\right)
\end{aligned}
$$




\section{Appendix B}

\section{Efficient Implementation of Simultaneous FFT}

$$
\begin{aligned}
& X 1_{r}(k)=\frac{1}{2}\left[X_{r}(k)+X_{r}(N-k)\right] \\
& X 2_{r}(k)=\frac{1}{2}\left[X_{i}(k)+X_{i}(N-k)\right] \\
& X 1_{r}(N-k)=X 1_{r}(k) \\
& X 2_{r}(N-k)=X 2_{r}(k) \\
& X 1_{i}(n)=\frac{1}{2}\left[X_{i}(k)-X_{i}(N-k)\right] \\
& X 1_{i}(n)=\frac{1}{2}\left[X_{r}(k)-X_{r}(N-k)\right] \\
& X 1_{i}(N-k)=-X 1_{i}(k) \\
& X 2_{i}(N-k)=-X 2_{i}(k)
\end{aligned}
$$

$$
\begin{aligned}
& X 1_{r}(0)=X_{r}(n) \\
& X 2_{r}(0)=X_{i}(n) \\
& X 1_{r}(N / 2)=X_{i}(N / 2) \\
& X 2_{r}(N / 2)=X_{i}(N / 2)
\end{aligned}
$$

$$
\begin{aligned}
& X 1_{i}(0)=0 \\
& X 2_{i}(0)=0 \\
& X 1_{i}(N / 2)=0 \\
& X 2_{i}(N / 2)=0
\end{aligned}
$$

